\title{
More than a Protective Group: Synthesis and Applications of a New Chiral Silane
}

\author{
Maurizio Campagna, Michael Trzoss, and Stefan Bienz* \\ * Institute of Organic Chemistry, University of Zurich \\ Winterthurerstrasse 190, CH-8057 Zürich \\ sbienz@oci.uzh.ch
}

1. Synthesis of MOTES-H $\quad$ S2

2. Nucleophilic additions to $\alpha$ - and $\beta$-silyloxyaldehydes $\quad$ S6

3. Diels-Alder reactions $\quad$ S15

$\begin{array}{ll}\text { 4. Enantioselective synthesis of (-)-frontalin } & \text { S21 }\end{array}$

$\begin{array}{lr}\text { 5. Enantioselective synthesis of }(\boldsymbol{R}) \text {-Octan-1,3-diol } & \text { S24 }\end{array}$

$\begin{array}{ll}\text { 6. MOTES as a chiral derivatizing agent } & \text { S25 }\end{array}$

7. Copies of the spectra $\quad$ S32 


\section{General remarks}

Unless otherwise stated, manipulations were carried out under $\mathrm{Ar}$ in oven-dried glass equipment. For reactions, $\mathrm{Et}_{2} \mathrm{O}$ and THF were freshly distilled from $\mathrm{Na}$ with benzophenone ketyl as indicator. Solns. for workup procedures were prepared in deionised $\mathrm{H}_{2} \mathrm{O}$. Solns. of $\mathrm{LiAlH}_{4}$ in $\mathrm{Et}_{2} \mathrm{O}(1.0 \mathrm{~m})$ commercially available (Sigma-Aldrich). Chromatography: Merck silica gel $60(40-63 \mu \mathrm{m})$. IR spectra: neat liquid films between $\mathrm{NaCl}$ plates; Perkin-Elmer IR "Spectrum One" and Perkin-Elmer 781; in $\mathrm{cm}^{-1} .{ }^{1} \mathrm{H}-\mathrm{NMR}$ spectra in $\mathrm{CDCl}_{3} ;$ Bruker AC-300 (300 MHz); $\delta$ in ppm rel. to $\mathrm{CHCl}_{3}(\delta 7.26), J$ in $\mathrm{Hz} .{ }^{13} \mathrm{C}-\mathrm{NMR}$ spectra in $\mathrm{CDCl}_{3} ;$ Bruker $A C$ $300(75.5 \mathrm{MHz}) ; \delta$ in ppm rel. to $\mathrm{CDCl}_{3}(\delta 77.0) ;{ }^{19} \mathrm{~F}-\mathrm{NMR}$ spectra in $\mathrm{CDCl}_{3}$ Bruker AC-300 (282.4 MHz); multiplicities from DEPT-135 and DEPT-90 experiments. ESI mass spectra were performed on a Bruker ESQUIRE-LC quadrupole ion trap instrument (Bruker Daltonik $G m b H$, Bremen, Germany), equipped with a combined Hewlett-Packard Atmospheric Pressure Ion (API) source (Hewlett-Packard Co., Palo Alto, CA, USA). EI and CI mass spectra were performed on a sector field mass analyzer (Finnigan MAT95, San Jose, CA; USA); the ionization energy was $70 \mathrm{eV}$ for $\mathrm{EI}$ and $150 \mathrm{eV}$ for $\mathrm{CI}$ with $\mathrm{NH}_{3}$ as reactant gas. Quasi-molecular ions and characteristic fragments in $m / z$ (rel. \%).

\section{Synthesis of MOTES-H}

\section{( \pm )-(1-Methoxy-2,2,2-triphenylethyl)(dimethyl)phenylsilane ( \pm )-(2)}

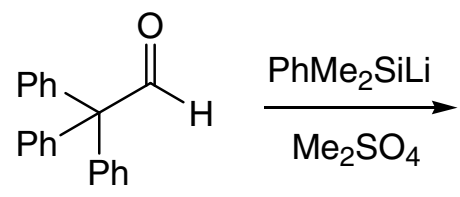

1<smiles>CO[C@@H](C(c1ccccc1)(c1ccccc1)c1ccccc1)[Si](C)(C)c1ccccc1</smiles>

$( \pm)-2$

Li (1.55 g, $252.43 \mathrm{mmol})$ was added to a soln. of $\mathrm{PhMe}_{2} \mathrm{SiCl}(14.2 \mathrm{~mL}, 85.51 \mathrm{mmol})$ in THF $(100 \mathrm{~mL})$ at $0{ }^{\circ} \mathrm{C}$ and the mixture was stirred for $8 \mathrm{~h} .{ }^{1}$ After this time, the soln. was transferred with a cannula into a second flask, containing a soln. of triphenylacetaldehyde $(\mathbf{1})^{2}(10.50 \mathrm{~g}, 50.51 \mathrm{mmol})$ in THF (200.0 $\mathrm{mL}$ ) at $-60{ }^{\circ} \mathrm{C}$, and the mixture was stirred for $30 \mathrm{~min}$ at $-30{ }^{\circ} \mathrm{C} . \mathrm{Me}_{2} \mathrm{SO}_{4}(20.5 \mathrm{~mL}, 216.2$ mmol) was then added and the stirring prolonged for $6 \mathrm{~h}$ at $23{ }^{\circ} \mathrm{C}$. The reaction was quenched with a sat. aq. soln. of $\mathrm{NH}_{4} \mathrm{Cl}(300 \mathrm{~mL})$, the ayers were separated, and the aq. phase was extracted with $\mathrm{Et}_{2} \mathrm{O}(2 \times 100 \mathrm{~mL})$. The combined organic layers were dried over $\mathrm{MgSO}_{4}$, and

\footnotetext{
${ }^{1}$ J. Buynak, J. B. Strickland, G. W. Lamb, D. Khasnis, S. Modi, J. Org. Chem. 1991, 56, 7076.

${ }^{2}$ F. A. Davis, R. E. Reddy, J. M. Szewczyk, G. V. Reddy, P. S. Portonovo, H. Zhang, D. Fanelli, R. T. Reddy, P. Zhou, P. J. Carroll, J. Org. Chem. 1997, 62, 2555.
} 
the solvent was removed under reduced pressure. The crude mixture was purified by recrystallization (hexane/EtOAc 50:1) to afford ( \pm )-2 as colorless crystals (2.20 g, $5.21 \mathrm{mmol}$, 84\%). M.p.: 59-61 ${ }^{\circ} \mathrm{C}$ (hexane). IR: 3050s, 2960s, 2920s, 2890s, 2810s, 1490s, 1445s, 1435s, 1245s, 1110s, 1090s, 1080s, 840s, 815s. ${ }^{1} \mathrm{H}-\mathrm{NMR}:$ 7.56-7.52, 7.37-7.14 (2m, 20 arom. H); $4.56(s, \mathrm{SiCH}) ; 3.10(s, \mathrm{MeO}) ;-0.10,-0.11$ ( $\left.2 s, \mathrm{Me}_{2} \mathrm{Si}\right) .{ }^{13} \mathrm{C}-\mathrm{NMR}: 145.9$ ( $s, 3$ arom. C); 140.6 (s, arom. C); 134.0, (d, 3 arom. C); 130.3 (d, 6 arom. C); 128.7, 127.7 (2d, 2 x 2 arom. C); 127.3 (d, 6 arom. C); 126.0 (d, arom. C); 83.5 (d, SiCH); $61.7\left(s, \mathrm{Ph}_{3} C\right) ; 61.5$ ( $\left.q, \mathrm{MeO}\right)$; $-0.3,-3.8\left(2 q, \mathrm{Me}_{2} \mathrm{Si}\right)$. CI-MS: $440\left(16,\left[M+\mathrm{NH}_{4}\right]^{+}\right) ; 243\left(100,\left[\mathrm{Ph}_{3} \mathrm{C}\right]^{+}\right)$.

\section{( \pm )-(1-Methoxy-2,2,2-triphenylethyl)(dimethyl)silane ( \pm )-(3)}

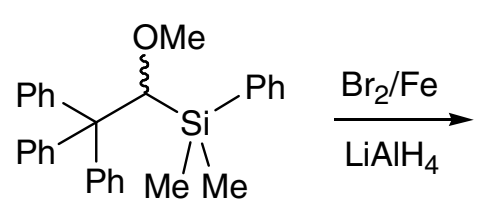

$( \pm)-2$<smiles>CO[C@H](C(c1ccccc1)(c1ccccc1)c1ccccc1)[Si](C)(C)C</smiles>

$( \pm)-3$

Fe (21.1 $\mathrm{mg}, 0.47 \mathrm{mmol})$ was added to a soln. of $( \pm)-2(4.22 \mathrm{~g}, 10.00 \mathrm{mmol})$ in $1,2-$ dichloroethane $(150.0 \mathrm{~mL})$. The mixtures was cooled to $-10{ }^{\circ} \mathrm{C}$ and $\mathrm{Br}_{2}(0.67 \mathrm{~mL}$, $10.30 \mathrm{mmol}$ ) was added dropwise. After complete consumption of the starting material (monitored by TLC, ca. $1.5 \mathrm{~h}$ ), a soln. of $\mathrm{LiAlH}_{4}\left(1.0 \mathrm{M}, 10.5 \mathrm{~mL}, 10.50 \mathrm{mmol}\right.$ ) in $\mathrm{Et}_{2} \mathrm{O}$ was added at $0{ }^{\circ} \mathrm{C}$, and it was stirred for $3 \mathrm{~h}$ at $23{ }^{\circ} \mathrm{C}$. The excess of $\mathrm{LiAlH}_{4}$ was neutralized with an aq. soln. of $\mathrm{H}_{2} \mathrm{SO}_{4}(10 \%)$, and the layers were separated. The aq. phase was extracted with $\mathrm{Et}_{2} \mathrm{O}(2 \times 100 \mathrm{~mL})$ and the combined organic layers were dried over $\mathrm{MgSO}_{4}$. Evaporation of the organic fraction and column chromatography $\left(\mathrm{SiO}_{2}\right.$; hexane/EtOAc 50:1) afforded ( \pm )-3 as a colorless viscous oil (3.29 g, $9.8 \mathrm{mmol}, 98 \%)$. IR: 3050s, 2960s, 2920s, 2875s, 2868s, 2140s, 1490s, 1445s, 1250s, 1095s, 880s. ${ }^{1} \mathrm{H}-\mathrm{NMR}: 7.28-7.13$ ( $m, 15$ arom. H); 4.41 (d, $J=1.0, \mathrm{SiCH}) ; 3.76(d s e p t, J=1.0,3.8, \mathrm{SiH}) ; 3.35(s, \mathrm{MeO}) ; 0.13,-0.44\left(2 d, J=3.8, \mathrm{Me}_{2} \mathrm{Si}\right)$.

${ }^{13}$ C-NMR: 145.9 ( $s, 3$ arom. C); 129.9 (d, 3 arom. C); 127.4 (d, 6 arom. C); 125.9 (d, 6 arom. C); $81.0(d, \mathrm{SiCH}) ; 61.2\left(s, \mathrm{Ph}_{3} C\right) ; 60.4$ (q, MeO); -2.0, -6.3 (2q, $\left.\mathrm{Me}_{2} \mathrm{Si}\right)$. ESI-MS: 369 (10, $\left.[M+\mathrm{Na}]^{+}\right) ; 303\left(100,[M-43]^{+}\right)$. 
(1'S,2S)- and (1'R,2S)-2-[(1-Methoxy-2,2,2-triphenylethyl)(dimethyl)silyloxy]-1,1,2-triphenylethanol (A1 and A2)

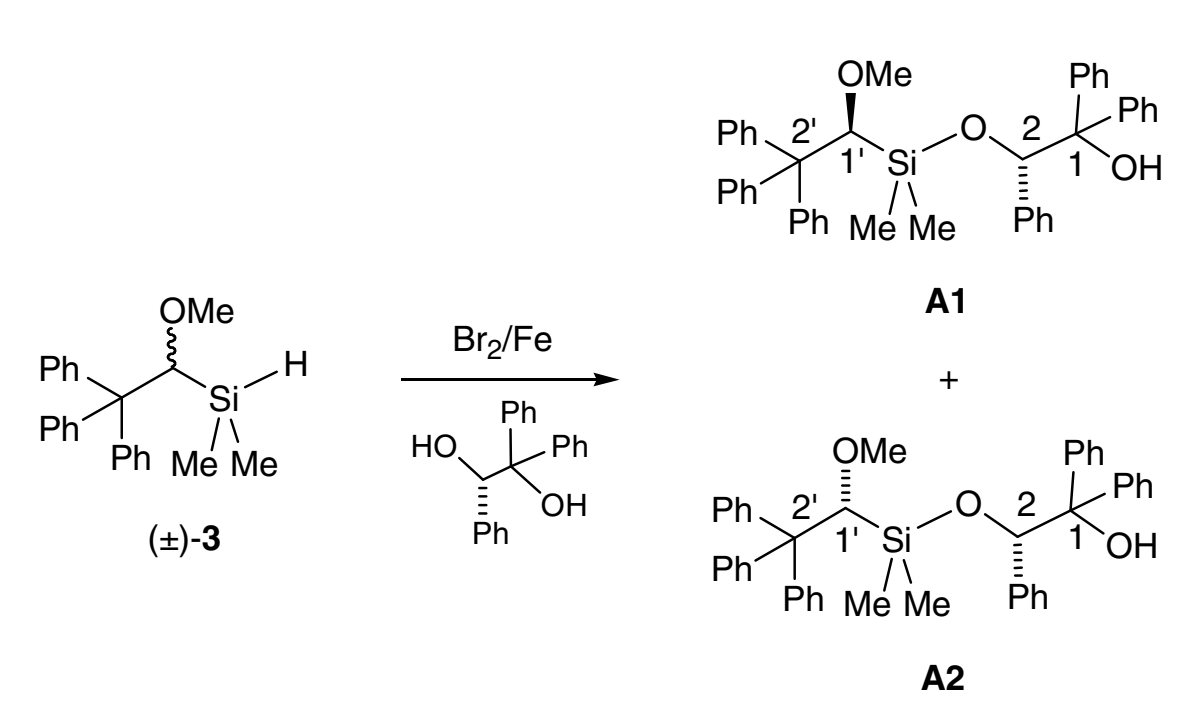

A soln. of ( \pm )-3 (3.46 g, $10.00 \mathrm{mmol})$ in $\mathrm{CH}_{2} \mathrm{Cl}_{2}$ $(150.0 \mathrm{~mL})$ was cooled to $-78{ }^{\circ} \mathrm{C}$ and $\mathrm{Br}_{2}(0.54$ $\mathrm{mL}, 10.05 \mathrm{mmol}$ ) was added dropwise. The mixture was stirred for $10 \mathrm{~min}$, the cooling bath was removed, and the solvent evaporated at 23

${ }^{\circ} \mathrm{C}$ at reduced pressure. The residue was dissolved in $\mathrm{CH}_{2} \mathrm{Cl}_{2}(200.0 \mathrm{~mL})$ and the mixture cooled to $0{ }^{\circ} \mathrm{C}$. $\mathrm{NEt}_{3}(2.8 \mathrm{~mL}, 20.00 \mathrm{mmol}),(S)$-1,1,2-triphenylethane-1,2-diol (3.21 g, 10.50 mmol), and DMAP (122.1 mg, $1.00 \mathrm{mmol}$ ) were added. The mixture was stirred at $23^{\circ} \mathrm{C}$ for 1 $\mathrm{h}$, quenched with $\mathrm{H}_{2} \mathrm{O}(100 \mathrm{~mL})$, and the two layers were separated. The aq. phase was extracted with $\mathrm{Et}_{2} \mathrm{O}(2 \times 200 \mathrm{~mL})$ and the combined organic layers were dried over $\mathrm{MgSO}_{4}$. Evaporation of the organic fraction and column chromatography $\left(\mathrm{SiO}_{2}\right.$; toluene) afforded $\mathbf{A 1}$ (200.8 mg, $0.36 \mathrm{mmol}, 36 \%)$ and subsequently A2 (217.3 $\mathrm{mg}, 0.37 \mathrm{mmol}, 38 \%)$ as colorless oils.

A1: $[\alpha]_{D}^{25}=-25.8\left(\mathrm{c}=1.23, \mathrm{CHCl}_{3}\right)$. IR: 3420s (br), 3050s, 2920s, 2860s, 2820s, 1490s, 1442s, 1250s, 1115s, 1095s, 1080s, 935s. ${ }^{1} \mathrm{H}-\mathrm{NMR}:$ 7.73-7.69, 7.39-6.97 (2m, 30 arom. H); $5.52(s, \mathrm{SiOCH}) ; 4.23$ (s, SiCH); 3.39 (br.s, OH); 3.07 (s, MeO); -0.25, -0.79 (2s, $\left.\mathrm{Me}_{2} \mathrm{Si}\right)$. ${ }^{13}$ C-NMR: 146.1, 145.8, 143.0, 139.6, 129.0, 128.2 (6s, 6 arom. C); 130.0, 128.8, 128.0, 127.5, 127.4, 127.3, (6d, 6 arom. C); 127.2, 127.0, 126.4, 126.3, 125.9, 125.3 (6d, 6 x 4 arom. C); $83.7(d, \mathrm{SiCH}) ; 81.0(s,(\mathrm{OH}) \mathrm{C}) ; 80.1(d, \mathrm{SiOCH}) ; 60.7\left(s, \mathrm{Ph}_{3} C\right) ; 60.6(q, \mathrm{MeO}) ; 0.0,-1.9$ $\left(2 q, \mathrm{Me}_{2} \mathrm{Si}\right)$. ESI-MS: $657\left([\mathrm{M}+\mathrm{Na}]^{+}\right)$.

A2: $[\alpha]_{D}^{25}=-156.2\left(\mathrm{c}=1.13, \mathrm{CHCl}_{3}\right)$. IR: 3420br.s, 3048s, 2920s, 2860s, 2820s, 1490s, 1440s, 1247s, 1114s, 1095s, 1080s, 935s. ${ }^{1} \mathrm{H}-\mathrm{NMR}:$ 7.80-7.75, 7.47-7.00 (2m, 30 arom. H); 5.55 ( $s, 1 \mathrm{H}, \mathrm{SiOCH}) ; 4.19(s, 1 \mathrm{H}, \mathrm{SiCH}) ; 3.47$ (br.s, 1H, OH); 3.11 (s, 3H, MeO); -0.40, -0.72 (2s, 6H, Me $2 \mathrm{Si}) .{ }^{13} \mathrm{C}-\mathrm{NMR}: 146.2,145.8,142.9,139.6,129.0,128.2$ (6s, 6 arom. C); 130.0, 128.8, 128.1, 127.4, 127.3, 127.1 (6d, 6 arom. C); 127.2, 127.0, 126.9, 126.4, 125.9, 
$125.3\left(6 d, 6\right.$ x 4 arom. C); $83.3(d, \mathrm{SiCH}) ; 81.0(s,(\mathrm{OH}) \mathrm{C}) ; 80.3(d, \mathrm{SiOCH}) ; 60.9\left(s, \mathrm{Ph}_{3} C\right)$; $60.5(q, \mathrm{MeO}) ;-0.3,-1.3\left(2 q, \mathrm{Me}_{2} \mathrm{Si}\right)$. ESI-MS: $657\left([M+\mathrm{Na}]^{+}\right)$.

The diastereomeric purities of $\mathbf{A 1}$ and $\mathbf{A 2}$ (> 99.9\%) were checked by ${ }^{1} \mathrm{H}-\mathrm{NMR}$.

\section{(+)-(S)-(1-Methoxy-2,2,2-triphenylethyl)(dimethyl)silane (+)-(S)-3}<smiles>COC(C([Si])(c1ccccc1)c1ccccc1)[Si](C)(OC(c1ccccc1)c1ccccc1)c1ccccc1</smiles>

A1<smiles>COC(C(c1ccccc1)(c1ccccc1)c1ccccc1)[Si](C)(C)C</smiles>

$(+)-3$

A soln. of A1 $(558.2 \mathrm{mg}, 1.00$ $\mathrm{mmol})$ in $\mathrm{CH}_{2} \mathrm{Cl}_{2}(15.0 \mathrm{~mL})$ was cooled to $\quad 0^{\circ} \mathrm{C}$. A soln. of $\mathrm{LiAlH}_{4}$ $(1.0 \mathrm{M}, 1.5 \mathrm{~mL}, 1.50 \mathrm{mmol})$ in $\mathrm{Et}_{2} \mathrm{O}$

was added and the mixture stirred for $1 \mathrm{~h}$ at $23{ }^{\circ} \mathrm{C}$. The excess of $\mathrm{LiAlH}_{4}$ was neutralized with an aq. soln. of $\mathrm{H}_{2} \mathrm{SO}_{4}(10 \%)$, the layers were separated and the aq. phase was extracted with $\mathrm{Et}_{2} \mathrm{O}(2 \times 50 \mathrm{~mL})$. The combined organic layers were dried over $\mathrm{MgSO}_{4}$, and the solvent was removed under reduced pressure. The crude mixture was purified by flash chromatography $\left(\mathrm{SiO}_{2}\right.$, hexane/EtOAc 50:1) to afford (+)-(S)-3 (341.7 mg, $\left.0.98 \mathrm{mmol}, 99 \%\right)$ as a colorless oil. $[\alpha]_{D}^{25}((S)-3)=+27.0\left(\mathrm{c}=0.98, \mathrm{CHCl}_{3}\right)$.

\section{(-)-(R)-(1-Methoxy-2,2,2-triphenylethyl)(dimethyl)silane (-)-(R)-3}<smiles>CO[C@@H](C(O)(c1ccccc1)c1ccccc1)C(c1ccccc1)(c1ccccc1)[N+](C)(C)c1ccccc1</smiles>

A2

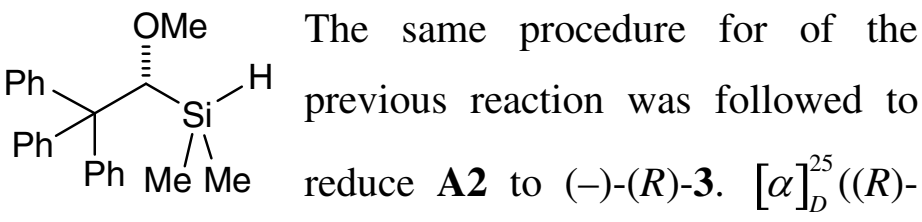

$(+)-3$
3) $=-30.1\left(\mathrm{c}=1.31, \mathrm{CHCl}_{3}\right)$.

\section{(S)-1-\{2-[(1-Methoxy-2,2,2-triphenylethyl)(dimethyl)silyloxy]phenyl\}ethanone (A3)}

\section{- Establishment of the absolute configuration of $(S)-3$.}<smiles>CO[C@H]([Si](C)(C)Oc1ccccc1C(C)=O)[Si](C)(OC)C(c1ccccc1)C(c1ccccc1)(c1ccccc1)c1ccccc1C(C)=O</smiles>

A soln. of $(S)-3(346.2 \mathrm{mg}, 1.0$ $\mathrm{mmol})$ in $\mathrm{CH}_{2} \mathrm{Cl}_{2}(15.0 \mathrm{~mL})$ was cooled to $-78{ }^{\circ} \mathrm{C}$, then $\mathrm{Br}_{2}$ (0.054 mL, $1.05 \mathrm{mmol})$ was added dropwise. The cooling

bath was removed and the solvent evaporated at $23{ }^{\circ} \mathrm{C}$ under reduced pressure. The residual was dissolved in $\mathrm{CH}_{2} \mathrm{Cl}_{2}(20.0 \mathrm{~mL})$ and the mixture cooled to $0{ }^{\circ} \mathrm{C}$. $\mathrm{NEt}_{3}(0.277 \mathrm{~mL}, 2.00$ 
mmol), 1-(2-hydroxyphenyl)ethanone (272.2 mg, $2.00 \mathrm{mmol})$, and DMAP (12.2 mg, 0.10 mmol) were added. The mixture was stirred at $23{ }^{\circ} \mathrm{C}$ for $1 \mathrm{~h}$, then quenched with $\mathrm{H}_{2} \mathrm{O}(10$ $\mathrm{mL})$. The two layers were separated and the aq. phase was extracted with $\mathrm{Et}_{2} \mathrm{O}(2 \times 20 \mathrm{~mL})$. The combined organic layers were dried over $\mathrm{MgSO}_{4}$ and the solvent was removed under reduced pressure. The crude mixture was purified by flash chromatography $\left(\mathrm{SiO}_{2}\right.$, hexane/EtOAc 15:1) to afford $\mathbf{A 3}$ as a colorless oil (413.4 mg, $0.86 \mathrm{mmol}, 86 \%$ ). Singlecrystal X-ray analysis confirmed the $(S)$ configuration for A3. M.p: $95.1-96.3{ }^{\circ} \mathrm{C}$ (hexane).

$[\alpha]_{D}^{25}(\mathbf{A 3})=+74.5\left(\mathrm{c}=0.98, \mathrm{CHCl}_{3}\right)$. IR: $3439 s, 3054 s, 1570 s, 1360 s, 1358 s, 1160 s, 1123 s$, 1030s, 960s, 880s. ${ }^{1} \mathrm{H}-\mathrm{NMR}: 7.63-6.83$ ( $m, 19$ arom. H); 4.65 ( $\left.s, \mathrm{SiCH}\right) ; 3.32(s, \mathrm{MeO}) ; 2.66$ $(s, \mathrm{MeCO}) ; 0.11,-0.19$ (2s, Me $2 \mathrm{Si}) .{ }^{13} \mathrm{C}-\mathrm{NMR}: 200.5$ ( $\left.s, \mathrm{MeCO}\right) ; 154.0$ (s, arom. C); 145.6 ( $s$, 3 arom. C); 132.9 ( $s$, arom. C); 131.6 (d, 3 arom. C); 130.1, 127.5 (2d, 2 x 6 arom. C); 126.2, 124.7, 121.3, 120.5 (4d, 4 arom. C); 83.3 (d, SiCH); 61.3 (q, MeO); $60.6\left(s, \mathrm{Ph}_{3} C\right) ; 31.1(q$, $M e \mathrm{CO}) ; 1.0,-1.4\left(2 q, \mathrm{Me}_{2} \mathrm{Si}\right)$. CI-MS: $481\left(5,[M+\mathrm{H}]^{+}\right) ; 193\left(100,\left[M+\mathrm{H}-\mathrm{C}_{21} \mathrm{H}_{20} \mathrm{O}\right]^{+}\right)$.

\section{Nucleophilic additions to $\alpha$ - and $\beta$-silyloxyaldehydes}

\subsection{Preparation of the substrates}

(1'R)-[(1-Methoxy-2,2,2-triphenylethyl)(dimethyl)silyloxy]ethanol (C1)<smiles>CO[C@@H]([Si](C)(C)C)[Si](C)(C)C(c1ccccc1)(c1ccccc1)c1ccccc1</smiles>

$(R)-\mathbf{3}$<smiles>OCCOCCOCCO</smiles>
$\mathrm{Et}_{3} \mathrm{~N}, \mathrm{DMAP}$<smiles>CO[Si](OCCO)(OCCO)C(c1ccccc1)(c1ccccc1)c1ccccc1</smiles>

C1 A soln. of $(R)-3(346.2 \mathrm{mg}, 1.0$ $\mathrm{mmol})$ in $\mathrm{CH}_{2} \mathrm{Cl}_{2}(15.0 \mathrm{~mL})$ was cooled to $-78{ }^{\circ} \mathrm{C}$, then $\mathrm{Br}_{2}$ (0.054 $\mathrm{mL}, 1.05 \mathrm{mmol})$ was

added drop wise. The cooling bath was removed, and the solvent evaporated under reduced pressure. The residual was dissolved in $\mathrm{CH}_{2} \mathrm{Cl}_{2}(20.0 \mathrm{~mL})$, the mixture cooled to $0{ }^{\circ} \mathrm{C}$, and $\mathrm{NEt}_{3}(0.28 \mathrm{~mL}, 2.0 \mathrm{mmol})$, ethylene glycol (124.4 mg, $\left.2 \mathrm{mmol}\right)$, and DMAP (12.2 mg, 0.01 mmol) were added. The mixture was stirred at $23{ }^{\circ} \mathrm{C}$ for $1 \mathrm{~h}$, then quenched with $\mathrm{H}_{2} \mathrm{O}(10$ $\mathrm{mL})$. The two layers were separated, the aq. phase was extracted with $\mathrm{Et}_{2} \mathrm{O}(2 \times 20 \mathrm{~mL})$, and the combined organic layers were dried over $\mathrm{MgSO}_{4}$. Evaporation of the organic fraction and column chromatography $\left(\mathrm{SiO}_{2}\right.$; hexane/EtOAc 15:1) afforded $\mathbf{C 1}$ as a colorless oil $(387.3 \mathrm{mg}$, $0.91 \mathrm{mmol}, 91 \%)$. IR: 3420br.s, 3050s, 2920s, 2860s, 2820s, 1490s, 1442s, 1250s, 1115s, 1095s, 1080s, 935s. ${ }^{1} \mathrm{H}-\mathrm{NMR}: 7.31-7.14$ (m, 15 arom. H); $4.41(s, 1 \mathrm{H}, \mathrm{SiCH}) ; 3.55-3.44$ (m, 
$\left.4 \mathrm{H}, \mathrm{SiOCH}_{2} \mathrm{CH}_{2}\right) ; 3.33(s, 3 \mathrm{H}, \mathrm{MeO}) ; 1.72(s, 1 \mathrm{H}, \mathrm{OH}) ;-0.06,-0.37\left(2 s, 6 \mathrm{H}, \mathrm{Me}_{2} \mathrm{Si}\right)$. ${ }^{13}$ C-NMR: 145.9 (s, arom. C); 130.1, $127.3,126.0$ (3d, arom. C); 83.1 (d, SiCH); 65.8, 63.6 $\left(2 t, \mathrm{SiOCH}_{2} \mathrm{CH}_{2}\right) ; 61.1(q, \mathrm{MeO}) ; 60.8\left(s, \mathrm{Ph}_{3} C\right) ;-1.0,-2.0\left(2 q, \mathrm{Me}_{2} \mathrm{Si}\right) .[\alpha]_{D}^{25}(\mathbf{C 1})=-74.1$ $\left(\mathrm{c}=1.25, \mathrm{CHCl}_{3}\right)$. CI-MS: $424\left(9,\left[M+\mathrm{NH}_{4}\right]^{+}\right) ; 257(100)$.

\section{(1'R)-[(1-Methoxy-2,2,2-triphenylethyl)(dimethyl)silyloxy]acetaldehyde (4)}<smiles>CO[C@@H](C(c1ccccc1)c1ccccc1)[C@](C)(OCCO)c1ccccc1</smiles>

C1<smiles>C=CCO[Si](C)(C)[C@H](OC)C(c1ccccc1)(c1ccccc1)c1ccccc1</smiles>

4

A soln. of $(\mathrm{COCl})_{2}(0.25$ $\mathrm{mL}, 2.95 \mathrm{mmol})$ in $\mathrm{CH}_{2} \mathrm{Cl}_{2}$ $(50.0 \mathrm{~mL})$ was cooled to $-78{ }^{\circ} \mathrm{C}$, then DMSO $(0.26$ $\mathrm{mL}, 3.69 \mathrm{mmol}$ ) added and the mixture stirred for $30 \mathrm{~min}$. After addition of a soln. of $\mathbf{C 1}(1.00 \mathrm{~g}, 2.46 \mathrm{mmol})$ in $\mathrm{CH}_{2} \mathrm{Cl}_{2}$ $(15.0 \mathrm{~mL})$, and 30 more min of stirring, $\mathrm{NEt}_{3}(1.1 \mathrm{~mL}, 7.87 \mathrm{mmol})$ was added, the temperature risen till $23{ }^{\circ} \mathrm{C}$ and the reaction quenched with $\mathrm{H}_{2} \mathrm{O}(30 \mathrm{~mL})$. The two layers were then separated, the aq. phase extracted with $\mathrm{Et}_{2} \mathrm{O}(2 \times 20 \mathrm{~mL})$, and the combined organic layers were dried over $\mathrm{MgSO}_{4}$. Evaporation of the organic fraction and column chromatography $\left(\mathrm{SiO}_{2}\right.$; hexane/EtOAc 10:1) afforded 4 (950.0 mg, $\left.2.35 \mathrm{mmol}, 95 \%\right)$ as a colorless dense oil. IR: 2920s, 2860s, 1748s, 1490s, 1445s, 1250s, 1125s, 1092s, 1080s, 935s. ${ }^{1} \mathrm{H}-\mathrm{NMR}: 9.54$ ( $\left.s, \mathrm{CHO}\right)$; 7.30-7.14 ( $m, 15$ arom. H); 4.45 ( $\left.s, 1 \mathrm{H}, \mathrm{SiCH}\right) ; 3.98$ ( $s, 2 \mathrm{H}$, $\left.\mathrm{SiOCH}_{2}\right) ; 3.33(s, 3 \mathrm{H}, \mathrm{MeO}) ;-0.12,-0.29\left(2 s, 6 \mathrm{H}, \mathrm{Me}_{2} \mathrm{Si}\right) .{ }^{13} \mathrm{C}-\mathrm{NMR}: 201.9$ (d, CHO); 145.9 ( $s, 3$ arom. C); 130.1, 127.4, 126.1 (3d, 15 arom. C); 83.7 (d, SiCH); $69.1\left(t, \mathrm{SiOCH}_{2}\right) ; 61.1$ $(q, \mathrm{MeO}) ; 60.7\left(s, \mathrm{Ph}_{3} C\right) ;-1.2,-1.5\left(2 q, \mathrm{Me}_{2} \mathrm{Si}\right) .[\alpha]_{D}^{25}(\mathbf{4})=-85.1\left(\mathrm{c}=1.20, \mathrm{CHCl}_{3}\right) . \mathrm{CI}-\mathrm{MS}:$ $438\left(16,\left[M+\mathrm{NH}_{4}\right]^{+}\right) ; 271(100)$. ESI-MS: $459\left(100,\left[M+\mathrm{CH}_{3} \mathrm{OH}+\mathrm{Na}\right]^{+}\right) ; 427\left(12,[M+\mathrm{Na}]^{+}\right)$.

\section{(1'R)-3-[(1-Methoxy-2,2,2-triphenylethyl)dimethylsilanoxy]propan-1-ol (D1)}<smiles>CO[C@@H]([Si](C)(C)C)[Si](C)(C)C(c1ccccc1)(c1ccccc1)c1ccccc1</smiles>

$(R)-3$

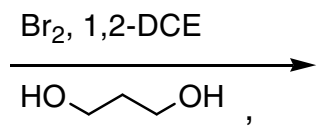
$\mathrm{Et}_{3} \mathrm{~N}, \mathrm{DMAP}$<smiles>CO[C@H](C(c1ccccc1)(c1ccccc1)c1ccccc1)[Si](C)(C)OCCCO</smiles>

D1

A soln. of (R)-3 (346.2 $\mathrm{mg}, \quad 1.0 \mathrm{mmol})$ in $\mathrm{CH}_{2} \mathrm{Cl}_{2}(15.0 \mathrm{~mL})$ was cooled to $-78{ }^{\circ} \mathrm{C}$, and $\mathrm{Br}_{2} \quad(0.054 \mathrm{~mL}, 1.05$ mmol) was added dropwise. The cooling bath was removed, and the solvent evaporated under reduced pressure. The residual was then dissolved in $\mathrm{CH}_{2} \mathrm{Cl}_{2}(20.0 \mathrm{~mL})$, the mixture cooled to $0{ }^{\circ} \mathrm{C}$ and $\mathrm{NEt}_{3}(0.28 \mathrm{~mL}, 2.0 \mathrm{mmol})$, propane-1,3-diol (152.2 mg, $\left.2 \mathrm{mmol}\right)$, and DMAP (12.2 
$\mathrm{mg}, 0.01 \mathrm{mmol}$ ) were added. The mixture was stirred at $23{ }^{\circ} \mathrm{C}$ for $1 \mathrm{~h}$ and quenched with $\mathrm{H}_{2} \mathrm{O}$ $(10 \mathrm{~mL})$. The two layers were separated, the aq. phase extracted with $\mathrm{Et}_{2} \mathrm{O}(2 \times 20 \mathrm{~mL})$, and the combined organic layers were dried over $\mathrm{MgSO}_{4}$. Evaporation of the organic fraction and column chromatography of the residue $\left(\mathrm{SiO}_{2}\right.$, hexane/EtOAc 15:1) afforded D1 as a colorless oil (403.2 mg, 0.96 mmol, 96\%). IR: 3417br.s, 3046s, 2931s, 2854s, 2829s, 1492s, 1444s, 1268s, 1106s, 1097s, 1071s, 941s. ${ }^{1} \mathrm{H}-\mathrm{NMR}: 7.35-7.09$ (m, 15 arom. H); 4.48 (s, 1H, SiCH); 4.20-3.96 (m, 4H, $\left.\mathrm{SiOCH}_{2} \mathrm{CH}_{2} \mathrm{CH}_{2} \mathrm{OH}\right) ; 3.67(s, 3 \mathrm{H}, \mathrm{MeO}) ; 2.41(s, 1 \mathrm{H}, \mathrm{OH}) ; 2.11-2.01$ (m, $\left.2 \mathrm{H}, \mathrm{SiOCH}_{2} \mathrm{CH}_{2}\right) ; 0.36,-0.01\left(2 s, 6 \mathrm{H}, \mathrm{Me}_{2} \mathrm{Si}\right) .{ }^{13} \mathrm{C}-\mathrm{NMR}: 147.8$ ( $s$, arom. C); 131.9, 129.2, 127.8 (3d, arom. C); 85.4 (d, $\mathrm{SiCH}) ; 63.6,63.5\left(2 t, \mathrm{SiOCH}_{2} \mathrm{CH}_{2} \mathrm{CH}_{2} \mathrm{OH}\right) ; 62.9(q, \mathrm{MeO}) ; 62.6$ $\left(s, \mathrm{Ph}_{3} C\right) ; 36.2\left(t, \mathrm{SiOCH}_{2} \mathrm{CH}_{2}\right) ;-1.0,-2.0\left(2 q, \mathrm{Me}_{2} \mathrm{Si}\right) .[\alpha]_{D}^{25}(\mathbf{D 1})=-72.8\left(\mathrm{c}=1.20, \mathrm{CHCl}_{3}\right)$. CI-MS: $438\left(16,\left[M+\mathrm{NH}_{4}\right]^{+}\right) ; 271(100)$.

\section{(1'R)-3-[(1-Methoxy-2,2,2-triphenylethyl)dimethylsilanoxy]propionaldehyde (5)}<smiles>CO[C@H](C(c1ccccc1)c1ccccc1)[Si](C)(C)OCCCO</smiles>

D1<smiles>CO[C@@H](C(c1ccccc1)(c1ccccc1)c1ccccc1)[C@](C)(OCCC=O)c1ccccc1</smiles>

5
A soln. of $(\mathrm{COCl})_{2}$ $(0.25 \mathrm{~mL}, \quad 2.95$ mmol) in $\mathrm{CH}_{2} \mathrm{Cl}_{2}$ $(50.0 \quad \mathrm{~mL}) \quad$ was cooled to $-78{ }^{\circ} \mathrm{C}$,

then DMSO $(0.26 \mathrm{~mL}, 3.69 \mathrm{mmol})$ added and the mixture stirred for $30 \mathrm{~min}$. After addition of a soln. of $\mathbf{D 1}(1.00 \mathrm{~g}, 2.46 \mathrm{mmol})$ in $\mathrm{CH}_{2} \mathrm{Cl}_{2}(15.0 \mathrm{~mL})$, and $30 \mathrm{~min}$ more of stirring at $-78{ }^{\circ} \mathrm{C}$, $\mathrm{NEt}_{3}(1.1 \mathrm{~mL}, 7.87 \mathrm{mmol})$ was added, and the temperature was allowed to rise to $23{ }^{\circ} \mathrm{C}$. The reaction was quenched with $\mathrm{H}_{2} \mathrm{O}(30 \mathrm{~mL})$, the two layers were separated, the aq. phase extracted with $\mathrm{Et}_{2} \mathrm{O}(2 \times 20 \mathrm{~mL})$, and the combined organic layers were dried over $\mathrm{MgSO}_{4}$. Evaporation of the organic fraction and column chromatography $\left(\mathrm{SiO}_{2}\right.$; hexane/EtOAc 10:1) afforded 5 (0.96 g, $2.36 \mathrm{mmol}, 96 \%)$ as a colorless oil. IR: 2918s, 2856s, 1753s, 1491s, 1443s, 1239s, 1129s, 1088s, 1081s, 941s. ${ }^{1} \mathrm{H}-\mathrm{NMR}: 9.76$ (s, CHO); $743-7.27$ (m, 15 arom. H); 4.51 $(s, 1 \mathrm{H}, \mathrm{SiCH}) ; 3.97-3.91\left(m, 2 \mathrm{H}, \mathrm{SiOCH}_{2}\right) ; 3.42(s, 3 \mathrm{H}, \mathrm{MeO}) ; 2.67-2.59\left(m, 2 \mathrm{H}, \mathrm{CH}_{2} \mathrm{CHO}\right)$ 0.17, -0.26 (2s, 6H, Me $2 \mathrm{Si}) .{ }^{13} \mathrm{C}-\mathrm{NMR}: 203.6$ (d, CHO); 147.8 (s, 3 arom. C); 131.9, 129.1, $127.8\left(3 d, 15\right.$ arom. C); $85.2(d, \mathrm{SiCH}) ; 62.7\left(t, \mathrm{SiOCH}_{2}\right) ; 62.5(q, \mathrm{MeO}) ; 58.5\left(s, \mathrm{Ph}_{3} C\right) ; 48.1$ $\left(t, \mathrm{CH}_{2} \mathrm{CHO}\right) ; 0.4,0.0$ (2q, Me $\left.\mathrm{Me}_{2} \mathrm{Si}\right)$. ESI-MS: $473\left(100,\left[M+\mathrm{CH}_{3} \mathrm{OH}+\mathrm{Na}\right]^{+}\right) ; 441$ (15, $\left.[M+\mathrm{Na}]^{+}\right) \cdot[\alpha]_{D}^{25}(\mathbf{5})=-81.6\left(\mathrm{c}=1.20, \mathrm{CHCl}_{3}\right) . \mathrm{CI}-\mathrm{MS}: 438\left(16,\left[M+\mathrm{NH}_{4}\right]^{+}\right) ; 271(100)$. 


\subsection{Addition reactions}

\section{General procedure for the stereospecific additions of Grignard reagents:}

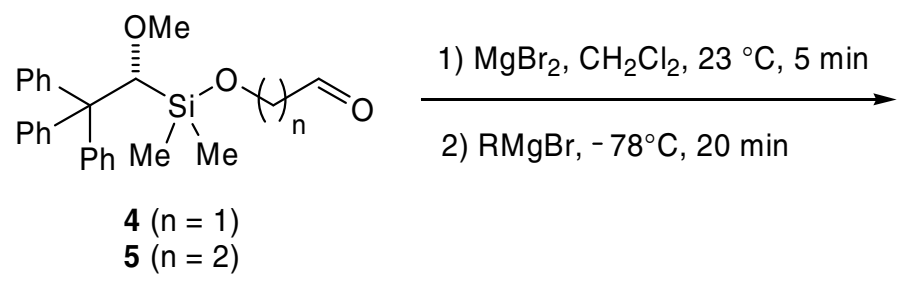

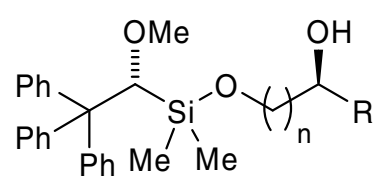

$8 \mathbf{a}-13 \mathbf{a}(\mathrm{n}=1)$

$14 a-19 a(n=2)$

(major)<smiles>[R]C(O)C(=C)O[Si](C)(C)C(OC)C(c1ccccc1)(c1ccccc1)c1ccccc1</smiles>

$8 b-13 b(n=1)$

$14 b-19 b(n=2)$

A soln. of $\mathrm{MgBr}_{2}$ in $\mathrm{Et}_{2} \mathrm{O}(1.00 \mathrm{M}, 0.40 \mathrm{~mL}, 0.40 \mathrm{mmol})$ was added to a soln. of 4 or $5(0.10$ mmol) in $\mathrm{CH}_{2} \mathrm{Cl}_{2}(1.00 \mathrm{~mL})$. The mixture was cooled to $-78{ }^{\circ} \mathrm{C}$ and a soln. of Grignard reagent in $\mathrm{CH}_{2} \mathrm{Cl}_{2}(1.0 \mathrm{M}, 0.30 \mathrm{~mL}, 0.30 \mathrm{mmol})$ was added dropwise. After $20 \mathrm{~min}$ the reaction was quenched with a sat. aq. soln. of $\mathrm{NH}_{4} \mathrm{Cl}(5 \mathrm{~mL})$. The two layers were separated, and the aq. phase was extracted with $\mathrm{Et}_{2} \mathrm{O}(2 \times 20 \mathrm{~mL})$. The combined organic layers were dried over $\mathrm{MgSO}_{4}$ and the solvent was removed under reduced pressure.

All the crude mixtures were analyzed by NMR spectroscopy and then purified by flash silica gel chromatography (hexane/EtOAc 10:1). Diastereoselectivities were established by integration of the signals correspondent to the $\mathrm{SiMe} 2$ of the two diastereoisomers' absorptions in ${ }^{1} \mathrm{H}-\mathrm{NMR}$. Analytical data refer to the major isomer.

$(1 ' R, 2 R)-$ and

(1'R,2S)-4-[(1-Methoxy-2,2,2-triphenylethyl)dimethylsilyloxy] propan-2-ol (8a and 8b)<smiles>CO[C@@H](C(C)(C)c1ccccc1)[C@](C)(OCC(C)=O)c1ccccc1</smiles>

$\mathbf{8 a}$ and $\mathbf{8 b}$

This compound was prepared according to the general procedure, adding $\mathrm{MeMgBr}$ to 4 (40.4 $\mathrm{mg}, 0.10 \mathrm{mmol}$ ). After purification, a mixture of $\mathbf{8 a}$ and $\mathbf{8 b}(41.7 \mathrm{mg}, 0.097 \mathrm{mmol}, 97 \%$, dr 70:1) was obtained as a colorless oil. IR: 3450br.m, 3050s, 2980s, 2920s, 2880s, 2820s, 1490s, 1445s, 1250s, 1095s, 1080s. ${ }^{1} \mathrm{H}-\mathrm{NMR}: 7.31-7.14$ (m, 15 arom. H); 4.41 $(s, 1 \mathrm{H}, \mathrm{SiCH}) ; 3.72-3.62,3.54-3.38,3.15-3.09$ (3m, 3H, $\left.\mathrm{SiOCH}_{2} \mathrm{CH}\right) ; 3.32(s, 3 \mathrm{H}, \mathrm{MeO})$; 2.07 (br.s, $1 \mathrm{H}, \mathrm{OH}) ; 1.03(d, J=6.3,3 \mathrm{H}, \mathrm{Me}(\mathrm{OH}) \mathrm{C}) ;-0.04,-0.40\left(2 s, 6 \mathrm{H}, \mathrm{Me}_{2} \mathrm{Si}\right) .{ }^{13} \mathrm{C}-\mathrm{NMR}$ : 145.9 (s, 3 arom. C); 130.1, 127.3, 126.0 (3d, 15 arom. C); $83.1(d, \mathrm{SiCH}) ; 68.1\left(t, \mathrm{SiOCH}_{2}\right)$; 
$67.8(d,(\mathrm{OH}) \mathrm{CH}) ; 61.1(q, \mathrm{MeO}) ; 60.8\left(s, \mathrm{Ph}_{3} C\right) ; 18.0(q, \mathrm{Me}(\mathrm{OH}) \mathrm{C}) ;-0.9,-2.4\left(2 q, \mathrm{Me}_{2} \mathrm{Si}\right)$. ESI-MS: $443\left([M+\mathrm{Na}]^{+}\right)$.

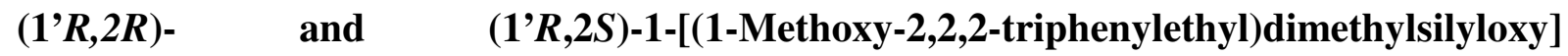
pentan-2-ol (9a and 9b)<smiles>CC[C@@H](O)CO[Si](C)(C)[C@@H](OC)C(c1ccccc1)(c1ccccc1)c1ccccc1</smiles>

$9 a$ and $9 b$

This compound was prepared according to the general procedure, adding EtMgBr to 4 (40.4 mg, $0.10 \mathrm{mmol}$ ). After purification, a mixture of 9a and 9b (41.6 mg, $0.095 \mathrm{mmol}, 95 \%$, dr 80:1) was obtained as a colorless oil. IR: 3450br.m, 3058s, 2960s, 2920s, $2885 s, 2820 s, 1595 s, 1490 s, 1448 s, 1250 s, 1095 s, 1080 s$.

${ }^{1} \mathrm{H}-\mathrm{NMR}: 7.32-7.15$ ( $m, 15$ arom. $\left.\mathrm{H}\right)$; 4.41 ( $\left.s, 1 \mathrm{H}, \mathrm{SiCH}\right) ; 3.56-3.39,3.24-3.16(2 m, 3 \mathrm{H}$, $\mathrm{SiOCH}_{2} \mathrm{CH}$ ); 3.34 (s, 3H, MeO); 2.06 (br.s, $\left.1 \mathrm{H}, \mathrm{OH}\right) ; 1.44-1.29$ ( $\left.m, 2 \mathrm{H}, \mathrm{MeCH}_{2}\right) ; 0.91(t$, $\left.J=7.4,3 \mathrm{H}, \mathrm{MeCH}_{2}\right) ;-0.03,-0.39$ (2s, 6H, Me $\left.2 \mathrm{Si}\right) .{ }^{13} \mathrm{C}-\mathrm{NMR}: 145.9$ (s, 3 arom. C); 130.1, 127.3, 126.0 (3d, 15 arom. C); $83.2(d, \mathrm{SiCH}) ; 73.0(d,(\mathrm{OH}) \mathrm{CH}), 66.4\left(t, \mathrm{SiOCH}_{2}\right) ; 61.2(q$, $\mathrm{MeO}) ; 60.8\left(s, \mathrm{Ph}_{3} C\right) ; 27.6\left(t, \mathrm{MeCH}_{2}\right) ; 9.9\left(q, \mathrm{MeCH}_{2}\right) ;-1.0,-2.2\left(2 q, \mathrm{Me}_{2} \mathrm{Si}\right)$. ESI-MS: 457 $\left([M+\mathrm{Na}]^{+}\right)$.

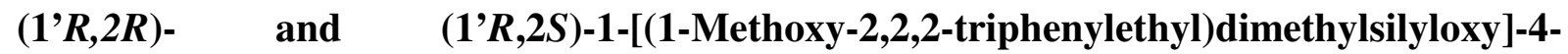
methylbutan-2-ol (10a and 10b)<smiles>CO[C@H]([C@H](OC)[Si](C)(C)c1ccccc1)C(C)(C)c1ccccc1</smiles>

$10 a$ and 10b

This compound was prepared according to the general procedure, adding $i \mathrm{PrMgBr}$ to 4 (40.4 mg, $0.10 \mathrm{mmol})$. After purification, a mixture of 10a and 10b (42.5 mg, $0.093 \mathrm{mmol}, 93 \%$, dr 70:1) was obtained as a colorless oil. IR: 3500br.m, 3058s, 2958s, 2920s, $2862 s, 1490 s, 1448 s, 1250 s, 1090 s, 1085 s .{ }^{1} \mathrm{H}-\mathrm{NMR}: 7.32-7.14$ ( $m, 15$ arom. H); $4.41(s, 1 \mathrm{H}, \mathrm{SiCH}) ; 3.47$ (dd, J= 2.9 (3.3), 9.5 (9.8), 1H, SiOCHHCH); 3.33 ( $s, 3 \mathrm{H}, \mathrm{MeO}$ ); 3.31-3.18 ( $m, 2 \mathrm{H}, \mathrm{SiOCH} \mathrm{HCH}$ und $(\mathrm{OH}) \mathrm{CH}$ ); 2.09 (br.s, 1H, OH); 1.67-1.51 $\left(m, 1 \mathrm{H}, \mathrm{Me}_{2} \mathrm{CH}\right) ; 0.94,0.85(2 d, J=6.8,6 \mathrm{H}, 2 \mathrm{MeCH}) ;-0.03,-0.38\left(2 s, 6 \mathrm{H}, \mathrm{Me}_{2} \mathrm{Si}\right)$. ${ }^{13}$ C-NMR: 145.9 (s, 3 arom. C); 130.1, 127.3, 126.0 (3d, 15 arom. C); 83.3 (d, SiCH); 76.4 (d, $(\mathrm{OH}) \mathrm{CH}), 64.9\left(t, \mathrm{SiOCH}_{2}\right) ; 61.1(q, \mathrm{MeO}) ; 60.7\left(s, \mathrm{Ph}_{3} C\right) ; 30.5\left(d, \mathrm{Me}_{2} \mathrm{CH}\right) ; 18.6,18.4(2 q, 2$ $\mathrm{MeCH}) ;-1.0,-2.1\left(2 q, \mathrm{Me}_{2} \mathrm{Si}\right)$. ESI-MS: $471\left([M+\mathrm{Na}]^{+}\right)$. 
$(1 ' R, 2 R)-\quad$ and $\quad(1 ' R, 2 S)-\quad$ [(1-Methoxy-2,2,2-triphenylethyl)dimethylsilyloxy $]-1-$ phenylethanol (11a and 11b)<smiles>CO[C@H](C(C)(c1ccccc1)c1ccccc1)[Si](C)(C)OC[C@H](O)c1ccccc1</smiles>

$11 \mathrm{a}$ and 11b

This compound was prepared according to the general procedure, adding $\mathrm{PhMgBr}$ to 4 (40.4 $\mathrm{mg}, 0.10 \mathrm{mmol}$ ). After purification, a mixture of 11a and 11b (44.2 mg, $0.096 \mathrm{mmol}, 96 \%$, dr 70:1) was obtained as a yellowish oil. IR: 3430br.m, 3050s, 2955s, 2920s, 2865s, 2850s, 1490s, 1448s, 1250s, 1105s, 1080s. ${ }^{1} \mathrm{H}-\mathrm{NMR}: 7.34-7.14$ ( $m, 15$ arom. H); 4.61-4.59 (m, 1H, $(\mathrm{OH}) \mathrm{CH}) ; 4.43(s, 1 \mathrm{H}, \mathrm{SiCH}) ; 3.61-3.51,3.37-3.31\left(2 m, 2 \mathrm{H}, \mathrm{SiOCH}_{2}\right)$; $3.32(s, 3 \mathrm{H}, \mathrm{MeO}) ; 2.62$ (br. $s, 1 \mathrm{H}, \mathrm{OH}) ;-0.09,-0.38\left(2 s, 6 \mathrm{H}, \mathrm{Me}_{2} \mathrm{Si}\right) .{ }^{13} \mathrm{C}-\mathrm{NMR}: 145.9,140.2$ (2s, 4 arom. C); 130.2, 128.2, 127.6, 127.4, 126.1, 126.0 (6d, 20 arom. C); 80.1 (d, SiCH); $74.1(d,(\mathrm{OH}) \mathrm{CH}) ; 68.4\left(t, \mathrm{SiOCH}_{2}\right) ; 61.2(q, \mathrm{MeO}) ; 60.7\left(s, \mathrm{Ph}_{3} C\right) ;-0.9,-2.2\left(2 q, \mathrm{Me}_{2} \mathrm{Si}\right)$. ESI-MS: $505\left([M+\mathrm{Na}]^{+}\right)$.

$(1 ' R, 2 R)$ - and (1'R,2S)-1-[(1-Methoxy-2,2,2-triphenylethyl)dimethylsilyloxy]pent-4-en-2ol (12a and 12b)<smiles>C=CC[C@H](O)CO[Si](C)(C)[C@H](OC)C(c1ccccc1)(c1ccccc1)c1ccccc1</smiles>

$12 a$ and $12 b$

This compound was prepared according to the general procedure, adding AllylMgBr to 4 (40.4 mg, $0.10 \mathrm{mmol})$. After purification, a mixture of 12a and 12b (42.1 mg, $0.088 \mathrm{mmol}, 88 \%$, dr 80:1) was obtained as colorless oil. IR: 3450br.m, 3050s, 2975s, 2920s, 2884s, 2820s, 1492s, 1448s, 1250s, 1098s, 1080s. ${ }^{1} \mathrm{H}-\mathrm{NMR}:$ 7.31-7.14 (m, 15 arom. $\mathrm{H}) ; 5.85-5.71\left(m, 1 \mathrm{H}, \mathrm{HC}=\mathrm{CH}_{2}\right) ; 5.11-5.03\left(m, 2 \mathrm{H}, \mathrm{HC}=\mathrm{CH}_{2}\right) ; 4.41$ $(s, 1 \mathrm{H}, \mathrm{SiCH}) ; 3.60-4.40,3.29-3.21\left(2 m, 3 \mathrm{H}, \mathrm{SiOCH}_{2} \mathrm{CH}\right) ; 3.32(s, 3 \mathrm{H}, \mathrm{MeO}) ; 2.18-2.05(m$, $3 \mathrm{H}, \mathrm{CH}_{2} \mathrm{C}=\mathrm{C}$ und $\left.\mathrm{OH}\right) ;-0.040,-0.045,\left(2 s, 6 \mathrm{H}, \mathrm{Me}_{2} \mathrm{Si}\right) .{ }^{13} \mathrm{C}-\mathrm{NMR}: 145.9$ ( $s, 3$ arom. C); $134.4\left(d, \mathrm{HC}=\mathrm{CH}_{2}\right) ; 130.1,127.3,126.0(3 d, 15$ arom. $\mathrm{C}) ; 117.2\left(t, \mathrm{HC}=\mathrm{CH}_{2}\right) ; 83.1(d, \mathrm{SiCH})$; 71.0, $70.9(d,(\mathrm{OH}) \mathrm{CH}) ; 66.1,66.0\left(2 t, \mathrm{SiOCH}_{2}\right) ; 61.1(q, \mathrm{MeO}) ; 60.7\left(s, \mathrm{Ph}_{3} C\right) ; 37.4(t$, $\left.\mathrm{CH}_{2} \mathrm{C}=\mathrm{C}\right) ;-1.0,-2.1\left(2 q, \mathrm{Me}_{2} \mathrm{Si}\right)$. ESI-MS: $469\left([M+\mathrm{Na}]^{+}\right)$.

$(1 ' R, 2 R)$ - and (1'R,2S)-5-[(1-Methoxy-2,2,2-triphenylethyl)dimethylsilyloxy]but-1-en-3ol (13a and 13b)<smiles>C=C[C@H](O)CO[Si](C)(C)[C@H](OC)C(c1ccccc1)(c1ccccc1)c1ccccc1</smiles>

13a and 13b
This compound was prepared according to the general procedure, adding VinylMgBr to 4 (40.4 $\mathrm{mg}, 0.10 \mathrm{mmol})$. After purification, a mixture of 13a and 13b (39.6 mg, $0.091 \mathrm{mmol}, 91 \%$, dr 70:1) was obtained as a colorless oil. IR: 3444br.m, 3039s, 2975s, 
2920s, 2878s, 2816s, 1491s, 1446s, 1249s, 1095s, 1080s. ${ }^{1} \mathrm{H}-\mathrm{NMR}: 7.69-7.50$ (m, 15 arom. $\mathrm{H})$; 6.29-6.14 (m, 1H, $\left.\mathrm{HC}=\mathrm{CH}_{2}\right) ; 5.13-5.49\left(m, 2 \mathrm{H}, \mathrm{HC}=\mathrm{CH}_{2}\right) ; 4.67(s, 1 \mathrm{H}, \mathrm{SiCH}) ; 4.12-3.97$ ( $\left.m, 3 \mathrm{H}, \mathrm{SiOCH}_{2} \mathrm{CH}\right) ; 3.68$ ( $\left.s, 3 \mathrm{H}, \mathrm{MeO}\right) ; 3.25$ (br. $\left.s, 3.12, \mathrm{OH}\right) ; 0.19,-0.01\left(2 s, 6 \mathrm{H}, \mathrm{Me}_{2} \mathrm{Si}\right.$ ). ${ }^{13} \mathrm{C}-\mathrm{NMR}: 145.9$ ( $s, 3$ arom. $\left.\mathrm{C}\right)$; $134.4\left(d, \mathrm{HC}=\mathrm{CH}_{2}\right)$; 130.1, 127.3, 126.0 (3d, 15 arom. C); $117.2\left(t, \mathrm{HC}=\mathrm{CH}_{2}\right) ; 83.1(d, \mathrm{SiCH}) ; 71.0,70.9(d,(\mathrm{OH}) \mathrm{CH}) ; 66.1,66.0\left(2 t, \mathrm{SiOCH}_{2}\right) ; 61.1(q$, $\mathrm{MeO}) ; 60.7\left(s, \mathrm{Ph}_{3} C\right) ;-1.0,-2.1\left(2 q, \mathrm{Me}_{2} \mathrm{Si}\right)$. ESI-MS: $455\left([M+\mathrm{Na}]^{+}\right)$.

$(1 ' R, 3 R)-\quad$ and (1'R,3S)-4-[(1-Methoxy-2,2,2-triphenylethyl)dimethylsilyloxy] butan-2-ol (14a and 14b)

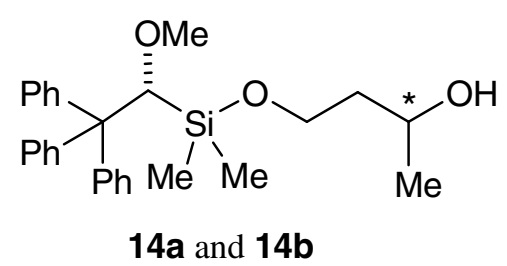

This compound was prepared according to the general procedure, adding $\mathrm{MeMgBr}$ to $5(41.8 \mathrm{mg}, 0.10 \mathrm{mmol})$. After purification, a mixture of $\mathbf{1 4 a}$ and $\mathbf{1 4 b}(41.7 \mathrm{mg}, 0.096 \mathrm{mmol}$, 96\%, dr 14:1) was obtained as a colorless oil. IR: 3439br.m, 3045s, 2976s, 2910s, 2876s, 2809s, 1488s, 1441s, 1247s, 1089s, 1077s. ${ }^{1} \mathrm{H}-\mathrm{NMR}: 7.46-7.31$ ( $m, 15$ arom. $\mathrm{H})$; $4.61(s, 1 \mathrm{H}, \mathrm{SiCH}) ; 4.18-4.09(m, 1 \mathrm{H}, \mathrm{CHOH}) ; 3.94-3.71\left(m, 2 \mathrm{H}, \mathrm{SiOCH}_{2}\right)$; 3.48 (s, 3H, MeO); 2.72 (br.s, 1H, OH); 1.73-1.67 ( $\left.m, 2 \mathrm{H}, \mathrm{CH}_{2} \mathrm{CHO}\right) ; 1.39-1.34$ (d, J=6.5, $3 \mathrm{H}, \mathrm{Me}(\mathrm{OH}) \mathrm{C}) ; 0.01,-0.13\left(2 s, 6 \mathrm{H}, \mathrm{Me}_{2} \mathrm{Si}\right) .{ }^{13} \mathrm{C}-\mathrm{NMR}: 145.2$ (s, 3 arom. C); 129.6, 128.1, $125.4\left(3 d, 15\right.$ arom. C); $83.1(d, \mathrm{SiCH}) ; 67.7\left(t, \mathrm{SiOCH}_{2}\right) ; 67.4(d,(\mathrm{OH}) \mathrm{CH}) ; 60.6(q, \mathrm{MeO})$; $60.3\left(s, \mathrm{Ph}_{3} C\right) ; 35.7\left(t, \mathrm{SiOCH}_{2} \mathrm{CH}_{2}\right) ; 16.9$ ( $\left.q, \mathrm{Me}(\mathrm{OH}) \mathrm{CH}\right) ;-0.1,-1.8\left(2 q, \mathrm{Me}_{2} \mathrm{Si}\right)$. ESI-MS: $457\left([M+\mathrm{Na}]^{+}\right)$.

$(1 ' R, 3 R)-\quad$ and (1'R,3S)-1-[(1-Methoxy-2,2,2-triphenylethyl)dimethylsilyloxy] hexan-3-ol (15a and 15b)<smiles>CC[C@@H](O)CCO[Si](C)(C)[C@@H](OC)C(c1ccccc1)(c1ccccc1)c1ccccc1</smiles>

15a and 15b
This compound was prepared according to the general procedure, adding EtMgBr to $5(41.8 \mathrm{mg}, 0.10 \mathrm{mmol})$. After purification, a mixture of $\mathbf{1 5 a}$ and $\mathbf{1 5 b}(42.6 \mathrm{mg}, 0.095 \mathrm{mmol}$, 95\%, dr 15:1) was obtained as a colorless oil. IR: 3456br.m, 3062s, 2971s, 2926s, 2891s, 2823s, 1588s, 1491s, 1455s, 1253s, 1099s, 1084s. ${ }^{1} \mathrm{H}-\mathrm{NMR}:$ 7.48-7.40 (m, 15 arom. H); $4.52(s, 1 \mathrm{H}, \mathrm{SiCH}) ; 3.98-3.74\left(m, 3 \mathrm{H}, \mathrm{SiOCH}_{2} \mathrm{CH}_{2} \mathrm{CH}\right) ; 3.49$ (s, $3 \mathrm{H}, \mathrm{MeO}$ ); 2.21 (br.s, 1H, OH); 1.81-1.75 (m, 2H, $\left.\mathrm{OCH}_{2} \mathrm{CH}_{2}\right) ; 1.70-1.62\left(m, 2 \mathrm{H}, \mathrm{MeCH}_{2}\right)$; 1.21-1.03 ( $m, 3 \mathrm{H}, \mathrm{MeCH}$ ); 0.01, -0.18 ( $\left.2 s, 6 \mathrm{H}, \mathrm{Me}_{2} \mathrm{Si}\right) .{ }^{13} \mathrm{C}-\mathrm{NMR}: 145.2$ ( $s, 3$ arom. C); 129.6, 128.1, 125.4 (3d, 15 arom. C); $83.6(d, \mathrm{SiCH}) ; 71.9(d,(\mathrm{OH}) \mathrm{CH}) ; 66.9\left(t, \mathrm{SiOCH}_{2}\right)$; 
61.8 (q, MeO); $61.5\left(s, \mathrm{Ph}_{3} C\right) ; 36.1\left(t, \mathrm{SiOCH}_{2} \mathrm{CH}_{2}\right) ; 28.4\left(t, \mathrm{MeCH}_{2}\right) ; 10.2\left(q, \mathrm{MeCH}_{2}\right) ;-1.0$, $-2.2\left(2 q, \mathrm{Me}_{2} \mathrm{Si}\right)$. ESI-MS: $471\left([\mathrm{M}+\mathrm{Na}]^{+}\right)$.

$\left(1^{\prime} R, 3 R\right)-\quad$ and $\quad(1 ' R, 3 S)-1-[(1-M e t h o x y-2,2,2-t$ triphenylethyl $)$ dimethylsilyloxy]-4methylpentan-3-ol (16a and 16b)<smiles>CO[C@@H](C(c1ccccc1)(c1ccccc1)c1ccccc1)[Si](C)(C)OCC[C@H](O)C(C)C</smiles>

$16 a$ and $16 b$

This compound was prepared according to the general procedure, adding $i \mathrm{PrMgBr}$ to $5(41.8 \mathrm{mg}, 0.10 \mathrm{mmol})$. After purification, a mixture of 16a and $\mathbf{1 6 b}(44.5 \mathrm{mg}, 0.096 \mathrm{mmol}$, 96\%, dr 11:1) was obtained as a colorless oil. IR: 3510br.m, $3064 s, 2962 s, 2927 s, 2865 s, 1490 s, 1456 s, 1254 s, 1094 s$, 1087s. ${ }^{1} \mathrm{H}-\mathrm{NMR}: 7.67-7.48$ ( $m, 15$ arom. H); 4.59 ( $\left.s, 1 \mathrm{H}, \mathrm{SiCH}\right) ; 4.28-3.99\left(m, 2 \mathrm{H}, \mathrm{SiOCH}_{2}\right)$; 3.88-3.79 (m, 1H, CHOH); 3.64 (s, 3H, MeO); 3.26 (br.s, 1H, OH); 2.09 (br.s, 1H, OH); 2.10-1.78 ( $\left.m, 3 \mathrm{H}, \mathrm{CH}_{2} \mathrm{CHCHMe}_{2}\right) ; 1.63-1.55\left(m, 1 \mathrm{H}, \mathrm{Me}_{2} \mathrm{CH}\right) ; 1.28,1.19(2 d, J=6.3,6 \mathrm{H}, 2$ $\mathrm{MeCH}$ ); 0.19, 0.01 (2s, 6H, Me $2 \mathrm{Si}) .{ }^{13} \mathrm{C}-\mathrm{NMR}: 146.9$ (s, 3 arom. C); 131.1, 128.3, 127.0 (3d, 15 arom. C); $84.6(d, \mathrm{SiCH}) ; 78.4(d,(\mathrm{OH}) \mathrm{CH}), 63.9\left(t, \mathrm{SiOCH}_{2}\right) ; 62.1(q, \mathrm{MeO}) ; 61.7(s$, $\left.\mathrm{Ph}_{3} C\right) ; 36.5\left(d, \mathrm{Me}_{2} \mathrm{CH}\right) ; 34.7\left(t, \mathrm{SiOCH}_{2} \mathrm{CH}_{2}\right) ; 19.4,18.7(2 q, 2 \mathrm{MeCH}) ;-0.8,-0.9$ (2q, $\left.\mathrm{Me}_{2} \mathrm{Si}\right)$. ESI-MS: $485\left([\mathrm{M}+\mathrm{Na}]^{+}\right)$.

$(1 ' R, 1 R)-\quad$ and $\quad(1 ' R, 1 S)-\quad$ [(1-Methoxy-2,2,2-triphenylethyl)dimethylsilyloxy]-1phenylpropanol (17a and 17b)<smiles>CO[C@@H](C(c1ccccc1)(c1ccccc1)c1ccccc1)[Si](C)(C)OCC[C@H](O)c1ccccc1</smiles>

$17 \mathrm{a}$ and $17 \mathrm{~b}$

This compound was prepared according to the general procedure, adding $\mathrm{PhMgBr}$ to 5 (41.8 $\mathrm{mg}, 0.10 \mathrm{mmol})$. After purification, a mixture of $\mathbf{1 7 a}$ and $\mathbf{1 7 b}(45.2 \mathrm{mg}, 0.091 \mathrm{mmol}$, 91\%, dr 92:8) was obtained as a yellowish oil. 17a was separated then from $\mathbf{1 7 b}$ by $\mathrm{SiO}_{2}$ chromatography on a preparative TLC (hexane/EtOAc 12:1). IR: 3428br.m, 3046s, 2950s, 2918s, 2867s, 2841s, 1483s, 1445s, 1247s, 1101s, 1076s. ${ }^{1} \mathrm{H}-\mathrm{NMR}$ : 7.63-7.48 (m, 20 arom. H); 5.11-5.07 ( $\left.m, 1 \mathrm{H},(\mathrm{OH}) \mathrm{CH}\right) ; 4.69$ ( $\left.s, 1 \mathrm{H}, \mathrm{SiCH}\right)$; 4.09$3.97\left(m, 2 \mathrm{H}, \mathrm{SiOCH}_{2}\right) ; 3.68$ ( $\left.s, 3 \mathrm{H}, \mathrm{MeO}\right) ; 2.47$ (br. $\left., 1 \mathrm{H}, \mathrm{OH}\right) ; 2.26-2.18(m, 2 \mathrm{H}$, $\mathrm{SiOCH}_{2} \mathrm{CH}_{2}$ ); 0.19, $0.02\left(2 s, 6 \mathrm{H}, \mathrm{Me}_{2} \mathrm{Si}\right) .{ }^{13} \mathrm{C}-\mathrm{NMR}: 144.2$ ( $s, 3$ arom. C); 138.8 ( $s, 1$ arom. C); 130.0, 128.4, 127.3, 127.0, 126.5, 126.2 (6d, 20 arom. C); 84.6 (d, SiCH); 74.1 (d, $(\mathrm{OH}) \mathrm{CH}) ; 68.4\left(t, \mathrm{SiOCH}_{2}\right) ; 61.2(q, \mathrm{MeO}) ; 60.7\left(s, \mathrm{Ph}_{3} C\right) ; 34.7\left(t, \mathrm{SiOCH}_{2} C_{2}\right) ;-1.0,-2.1$ $\left(2 q, \mathrm{Me}_{2} \mathrm{Si}\right) . \mathrm{ESI}-\mathrm{MS}: 519\left([\mathrm{M}+\mathrm{Na}]^{+}\right)$. 
$(1 ' R, 3 R)$ - and (1'R,3S)-1-[(1-Methoxy-2,2,2-triphenylethyl)dimethylsilyloxy]hex-5-en-3ol (18a and 18b)<smiles>C=CC[C@H](O)CCO[Si](C)(C)[C@H](OC)C(c1ccccc1)(c1ccccc1)c1ccccc1</smiles>

This compound was prepared according to the general procedure, adding AllylMgBr to 5 (41.8 $\mathrm{mg}, 0.10 \mathrm{mmol})$. After purification, a mixture of $\mathbf{1 8 a}$ and $\mathbf{1 8 b}(45.1 \mathrm{mg}, 0.098$ mmol, 98\%, dr 16:1) was obtained as colorless oil. IR: 3452br.m, 3053s, 2979s, 2924s, 2887s, 2823s, 1495s, 1451s, 1253s, 1103s, 1084s. ${ }^{1} \mathrm{H}-\mathrm{NMR}: 7.48-7.31$ ( $m, 15$ arom. H); 6.10-5.94 ( $\left.m, 1 \mathrm{H}, \mathrm{HC}=\mathrm{CH}_{2}\right)$; 5.37-5.26 $\left(m, 2 \mathrm{H}, \mathrm{HC}=\mathrm{CH}_{2}\right) ; 4.55(s, 1 \mathrm{H}, \mathrm{SiCH}) ; 4.06-3.81\left(m, 3 \mathrm{H}, \mathrm{SiOCH}_{2} \mathrm{CH}_{2} \mathrm{CH}\right) ; 3.50(s$, $3 \mathrm{H}, \mathrm{MeO}$ ); 3.10 (br. $s, 3.12, \mathrm{OH}) ; 2.45-2.38\left(m, 2 \mathrm{H}, \mathrm{CH}_{2} \mathrm{CH}=\mathrm{CH}_{2}\right) ; 1.74-1.35(m, 2 \mathrm{H}$, $\left.\mathrm{SiOCH}_{2} \mathrm{CH}_{2}\right) ; 0.02,-0.16\left(2 s, 6 \mathrm{H}, \mathrm{Me}_{2} \mathrm{Si}\right) .{ }^{13} \mathrm{C}-\mathrm{NMR}: 146.1$ ( $s, 3$ arom. C); 134.8 (d, $\left.\mathrm{HC}=\mathrm{CH}_{2}\right) ; 130.1,127.3,126.0(3 d, 15$ arom. $\mathrm{C}) ; 117.1\left(t, \mathrm{HC}=\mathrm{CH}_{2}\right) ; 83.6(d, \mathrm{SiCH}) ; 70.8(d$, $(\mathrm{OH}) \mathrm{CH}) ; 63.1\left(t, \mathrm{SiOCH}_{2}\right) ; 62.9(q, \mathrm{MeO}) ; 62.7\left(s, \mathrm{Ph}_{3} C\right) ; 37.4\left(t, C \mathrm{H}_{2} \mathrm{C}=\mathrm{C}\right) ; 35.6(t$, $\left.\mathrm{SiOCH}_{2} \mathrm{CH}_{2}\right) ; 0.0,-0.1\left(2 q, \mathrm{Me}_{2} \mathrm{Si}\right)$. ESI-MS: $483\left([M+\mathrm{Na}]^{+}\right)$.

$(1 ' R, 3 R)$ - and (1'R,3S)-5-[(1-Methoxy-2,2,2-triphenylethyl)dimethylsilyloxy]pent-1-en-3ol (19a and 19b)<smiles>C=C[C@H](O)CCO[Si](C)(C)[C@H](OC)C(c1ccccc1)(c1ccccc1)c1ccccc1</smiles>

$19 a$ and $19 b$

This compound was prepared according to the general procedure, adding VinylMgBr to 5 (41.8 $\mathrm{mg}, 0.10 \mathrm{mmol})$. After purification, a mixture of 19a and 19b $(41.5 \mathrm{mg}, 0.093 \mathrm{mmol}$, 93\%, dr 16:1) was obtained as a colorless oil. IR: 3446br.m, $3041 s, 2975 s, 2920 s, 2878 s, 2816 s, 1491 s, 1446 s, 1249 s$, 1095s, 1080s. ${ }^{1} \mathrm{H}-\mathrm{NMR}: 7.67-7.47$ (m, 15 arom. H); 6.29-6.14 (m, 1H, HC= $\left.\mathrm{CH}_{2}\right)$; 5.13-5.49 $\left(m, 2 \mathrm{H}, \mathrm{HC}=\mathrm{CH}_{2}\right) ; 4.67(s, 1 \mathrm{H}, \mathrm{SiCH}) ; 4.12-3.97\left(m, 3 \mathrm{H}, \mathrm{SiOCH}_{2} \mathrm{CH}_{2} \mathrm{CH}\right) ; 3.68(s, 3 \mathrm{H}$, $\mathrm{MeO}$ ); 3.25 (br. $s, 3.12, \mathrm{OH}) ; 2.09-1.94\left(m, 2 \mathrm{H}, \mathrm{SiOCH}_{2} \mathrm{CH}_{2}\right) ; 0.19,-0.01\left(2 s, 6 \mathrm{H}, \mathrm{Me}_{2} \mathrm{Si}\right)$. ${ }^{13}$ C-NMR: 147.8 (s, 3 arom. C); $142.7\left(d, \mathrm{HC}=\mathrm{CH}_{2}\right)$; 131.8, 129.5, 127.9 (3d, 15 arom. C); $116.2\left(t, \mathrm{HC}=C \mathrm{H}_{2}\right) ; 85.6(d, \mathrm{SiCH}) ; 73.7(d,(\mathrm{OH}) \mathrm{CH}) ; 63.0(q, \mathrm{MeO}) ; 62.7\left(t, \mathrm{SiOCH}_{2}\right) ; 62.7$ $\left(s, \mathrm{Ph}_{3} C\right) ; 40.4\left(t, \mathrm{SiOCH}_{2} \mathrm{CH}_{2}\right) ; 0.9,0.0\left(2 q, \mathrm{Me}_{2} \mathrm{Si}\right)$. ESI-MS: $469\left([\mathrm{M}+\mathrm{Na}]^{+}\right)$. 


\subsection{Stereochemical assignments ${ }^{3}$}

(R)-1-Phenylpropane-1,3-diol (E1)<smiles>OCCC(O)c1ccccc1</smiles><smiles>CO[C@@H](C(c1ccccc1)(c1ccccc1)c1ccccc1)[Si](C)(C)OCCC(O)c1ccccc1</smiles>

17a

E1
A soln. of $\mathrm{LiAlH}_{4}$ in $\mathrm{Et}_{2} \mathrm{O}$ (1.0 M, $0.3 \mathrm{~mL}, 0.30 \mathrm{mmol}$ ) was added to a soln. of $\mathbf{1 7 a}(99.2 \mathrm{mg}$, 0.20 mmol, de $>99.9 \%$ checked by $\left.{ }^{1} \mathrm{H}-\mathrm{NMR}\right)$ in THF $(1.0 \mathrm{~mL})$ at $0{ }^{\circ} \mathrm{C}$. After $30 \mathrm{~min}$, a sat. aq.<smiles>CO[C@@H](c1ccccc1)[Si](C)(C)C(c1ccccc1)(c1ccccc1)c1ccccc1</smiles>

$(R)-3$ soln. of $\mathrm{NH}_{4} \mathrm{Cl}(2 \mathrm{~mL})$ was added, the two layers were separated and the aq. phase was extracted with $\mathrm{Et}_{2} \mathrm{O}(2 \times 5 \mathrm{~mL})$.

The combined organic layers were dried over $\mathrm{MgSO}_{4}$ and the solvent was removed under reduced pressure. Purification by flash chromatography $\left(\mathrm{SiO}_{2}\right.$; hexane/EtOAc 40:1 to 5:1) afforded $(R)$-MOTES-H $(R)-3(67.8 \mathrm{mg}, 0.196 \mathrm{mmol}, 96 \%)$ and subsequently E1 (29.8 mg, $0.19 \mathrm{mmol}, 96 \%)$ as a slightly yellowish oil. $[\alpha]_{D}^{25}=+66.3\left(\mathrm{c}=0.90, \mathrm{CHCl}_{3}\right)\left[(R)-\mathbf{E 1}:[\alpha]_{D}^{25}\right.$ $\left.=+69.0 \quad\left(\mathrm{c}=1.00, \mathrm{CHCl}_{3}\right), \quad(S)-\mathbf{E} 1:[\alpha]_{D}^{25}=-63.3 \quad\left(\mathrm{c}=1.0, \mathrm{CHCl}_{3}\right)\right]^{4}$, confirming the configuration of $\mathbf{1 7} \mathbf{a}$ as shown above. Analytical data in agreement with literature. ${ }^{5}$

\section{Diels-Alder reactions}

\subsection{Preparation of the substrates}

(1’R)-1-[(1-Methoxy-2,2,2-triphenylethyl)dimethylsilyloxy]pent-3-en-2-ol (F1)<smiles>CO[C@@H](C(c1ccccc1)(c1ccccc1)c1ccccc1)[Si](C)(C)OCC=O</smiles>

4<smiles>CC=CCl</smiles><smiles>CO[C@H](C([Si])(c1ccccc1)c1ccccc1)[Si](C)(OC)OC</smiles>

F1

Lithium $\mathrm{mg}, \quad 80.0 \mathrm{mmol})$ was added to a soln. of trans-1bromopropene $(0.69 \mathrm{~mL}, 8.0 \mathrm{mmol}, E / Z>99 \%)$ in $\mathrm{Et}_{2} \mathrm{O}(50.0 \mathrm{~mL})$ at $0{ }^{\circ} \mathrm{C}$ and stirred for $2 \mathrm{~h}$.

\footnotetext{
${ }^{3}$ The establishment of the configuration of the addition products by deprotection of $\mathbf{1 7} \mathbf{a}$ and correlation to diol E1, was supported by derivatization of samples 14a-19a with $(R)$-Mosher acid chloride and NMR-spectroscopic analysis (J. A. Dale, H. S. Mosher, J. Am. Chem. Soc. 1972, 95, 512)

${ }^{4}$ R. Chenevert, G. Fortier, R. B. Rhlid, Tetrahedron 1992, 48, 6769.

${ }^{5}$ Y. Gao, K. B. Sharpless, J. Org. Chem. 1988, 53, 4081.
} 
The liquid phase was added to a soln. of $4(800.5 \mathrm{mg}, 1.99 \mathrm{mmol})$ in $\mathrm{Et}_{2} \mathrm{O}(50.0 \mathrm{~mL})$ at $-78{ }^{\circ} \mathrm{C}$, the reaction warmed up to $-50{ }^{\circ} \mathrm{C}$ and stirred prolonged for $1 \mathrm{~h}$. The cooling bath was removed, and $\mathrm{H}_{2} \mathrm{O}(30 \mathrm{~mL})$ added. The two layers were separated, the aq. phase was extracted with $\mathrm{Et}_{2} \mathrm{O}(2 \times 50 \mathrm{~mL})$, and the combined organic layers were dried over $\mathrm{MgSO}_{4}$. Evaporation of the organic fraction and column chromatography $\left(\mathrm{SiO}_{2}\right.$; hexane/EtOAc 15:1) afforded $\mathbf{F 1}$ (781.9 mg, 1.75 mmol, 88\%). IR: 3448br.m, 2960s, 2928s, 1490s, 1447s, 1452s, 1245s, 1090s, 1078s, 822s. CI-MS: 271 (41); 106 (100). The NMR-data refer to the major isomer. ${ }^{1} \mathrm{H}-\mathrm{NMR}: 7.38-7.12(m, 15$ arom. $\mathrm{H})$; 5.81-5.50 ( $\left.m, 1 \mathrm{H}, \mathrm{HC}=\mathrm{CHCH}_{3}\right) ; 5.42-5.30(m, 1 \mathrm{H}$, $\left.\mathrm{HC}=\mathrm{CHCH}_{3}\right) ; 4.45(s, 1 \mathrm{H}, \mathrm{SiCH}) ; 4.08-3.92(m, 1 \mathrm{H},(\mathrm{OH}) \mathrm{CH}) ; 3.50-3.42\left(m, 2 \mathrm{H}, \mathrm{SiOCH}_{2}\right)$; $3.36(s, 3 \mathrm{H}, \mathrm{MeO}) ; 2.30(s, 1 \mathrm{H}, \mathrm{OH}) ; 1.67-1.58\left(m, 3 \mathrm{H}, \mathrm{CH}_{3} \mathrm{CH}\right) ;-0.03,-0.39(s, 6 \mathrm{H}$, $\left.\mathrm{Me}_{2} \mathrm{Si}\right) .{ }^{13} \mathrm{C}-\mathrm{NMR}: 146.8$ ( $s, 3$ arom. C); $131.1\left(d, \mathrm{HC}=\mathrm{CHCH}_{3}\right) ; 129.4,128.5,127.1(3 d, 15$ arom. C); $126.9\left(t, \mathrm{HC}=\mathrm{CHCH}_{3}\right) ; 84.0(d, \mathrm{SiCH}) ; 73.4(d,(\mathrm{OH}) \mathrm{C}) ; 67.7\left(t, \mathrm{SiOCH}_{2}\right) ; 62.1(q$, $\mathrm{MeO}) ; 61.7\left(s, \mathrm{Ph}_{3} C\right) ; 18.7\left(q, \mathrm{CH}_{3} \mathrm{CH}\right) ;-0.1,-1.2\left(2 q, \mathrm{Me}_{2} \mathrm{Si}\right)$.

\section{(1'R)-1-[(1-Methoxy-2,2,2-triphenylethyl)(dimethyl)silyloxy]pent-3-en-2-on (7)}<smiles>C/C=C/[C@@H](O)CO[Si](C)(C)[C@H](OC)C(c1ccccc1)(c1ccccc1)c1ccccc1</smiles>

F1

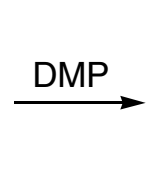<smiles>C/C=C/C(=O)CO[Si](C)(C)[C@H](OC)C(c1ccccc1)(c1ccccc1)c1ccccc1</smiles>

7

DMP $(2.2 \mathrm{~mL}$ of a $15 \%$ soln. in $\mathrm{CH}_{2} \mathrm{Cl}_{2}, 1.02$ mmol) was added to a soln. of a mixture of $\mathbf{F 1}(303.2 \mathrm{mg}, 0.68 \mathrm{mmol})$ in $\mathrm{CH}_{2} \mathrm{Cl}_{2}(7.0 \mathrm{~mL})$ at $0{ }^{\circ} \mathrm{C}$. After $10 \mathrm{~min}$, the mixture was allowed to warm to $20^{\circ} \mathrm{C}$ and stirred for $22 \mathrm{~h}$. Then, $\mathrm{MeOH}(0.3 \mathrm{~mL})$ was added. Evaporation of the combined organic fractions and column chromatography $\left(\mathrm{SiO}_{2}\right.$; hexane/EtOAc 20:1) afforded 7 (289.8 $\mathrm{mg}, 0.65 \mathrm{mmol}, 96 \%)$ as a yellowish oil. IR: $2958 \mathrm{~s}$, 2924s, 1740s, 1700s, 1490s, 1445s, 1456s, 1250s, 1095s, 1080s, 830s. ${ }^{1} \mathrm{H}-\mathrm{NMR}: 7.31-7.14$ $(m, 15$ arom. $\mathrm{H}) ; 6.50\left(d d, J=10.3,17.3,1 \mathrm{H}, H \mathrm{C}=\mathrm{CH}_{2}\right) ; 6.27(d d, J=2.0,17.3$, $1 \mathrm{H}, \mathrm{HC}=\mathrm{CHH}) ; 5.74(d d, J=2.0,10.3,1 \mathrm{H}, \mathrm{HC}=\mathrm{CH} H) ; 4.46(s, 1 \mathrm{H}, \mathrm{SiCH}) ; 4.16\left(s, \mathrm{SiOCH}_{2}\right)$; $3.34(s, 3 \mathrm{H}, \mathrm{MeO}) ;-0.11,-0.32\left(2 s, 6 \mathrm{H}, \mathrm{Me}_{2} \mathrm{Si}\right) .{ }^{13} \mathrm{C}-\mathrm{NMR}: 198.3(s, \mathrm{CO}) ; 146.0$ ( $s, 3$ arom. C); $131.9\left(d, \mathrm{HC}=\mathrm{CH}_{2}\right) ; 130.1,127.4,126.0\left(3 d, 15\right.$ arom. C); $128.6\left(t, \mathrm{HC}=\mathrm{CH}_{2}\right) ; 83.7(d$, $\mathrm{SiCH}) ; 67.9\left(t, \mathrm{SiOCH}_{2}\right) ; 61.1(q, \mathrm{MeO}) ; 60.7\left(s, \mathrm{Ph}_{3} C\right) ;-1.3,-1.6\left(2 q, \mathrm{Me}_{2} \mathrm{Si}\right) .[\alpha]_{D}^{25}(7)=$ $-69.5\left(\mathrm{c}=1.15, \mathrm{CHCl}_{3}\right)$. ESI-MS: $453\left([\mathrm{M}+\mathrm{Na}]^{+}\right)$. 
(1'R)-[(1-Methoxy-2,2,2-triphenylethyl)(dimethyl)silyloxy]but-3-en-2-ol (G1)<smiles>CO[C@@H]([Si](C)(C)C)[Si](C)(C)C(c1ccccc1)(c1ccccc1)c1ccccc1</smiles>

$(R)-3$
mmol) was add

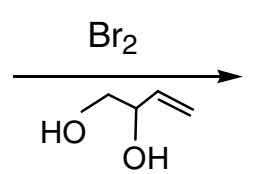

reduced pressure. The residual was dissolved in $\mathrm{CH}_{2} \mathrm{Cl}_{2}(20.0 \mathrm{~mL})$, the mixture cooled to 0 ${ }^{\circ} \mathrm{C}$, and $\mathrm{NEt}_{3}(0.28 \mathrm{~mL}, 2.0 \mathrm{mmol})$, 3-Buten-1,2-diol (176.6 mg, $2 \mathrm{mmol}$ ), and DMAP (12.2 $\mathrm{mg}, 0.01 \mathrm{mmol})$ added. The mixture was stirred at $23{ }^{\circ} \mathrm{C}$ for $1 \mathrm{~h}$, then quenched with $\mathrm{H}_{2} \mathrm{O}(10$ $\mathrm{mL})$. The two layers were separated, the aq. phase extracted with $\mathrm{Et}_{2} \mathrm{O}(2 \times 20 \mathrm{~mL})$, and the combined organic layers dried over $\mathrm{MgSO}_{4}$. Evaporation of the organic fraction, and column chromatography $\left(\mathrm{SiO}_{2}\right.$; hexane/EtOAc 15:1) afforded G1 as a colorless oil (398.0 mg, 0.92 mmol, 92\%). IR: 3450br.m, 2960s, 2922s, 1490s, 1455s, 1456s, 1250s, 1090s, 1080s, 830s. CI-MS: 257 (59); 92 (100). The NMR-data refer to the major isomer. ${ }^{1} \mathrm{H}-\mathrm{NMR}: 7.31-7.14$ ( $m$, 15 arom. $\mathrm{H}) ; 5.78-5.66\left(m, 1 \mathrm{H}, \mathrm{HC}=\mathrm{CH}_{2}\right) ; 5.32-5.12\left(m, 2 \mathrm{H}, \mathrm{HC}=\mathrm{CH}_{2}\right) ; 4.42(s, 1 \mathrm{H}, \mathrm{SiCH})$; 4.06-3.97 (m, 1H, (OH)CH); 3.50-3.42 ( $\left.m, 2 \mathrm{H}, \mathrm{SiOCH}_{2}\right) ; 3.32(s, 3 \mathrm{H}, \mathrm{MeO}) ; 2.26(s, 1 \mathrm{H}$, $\mathrm{OH}) ;-0.04,-0.38\left(2 s, 6 \mathrm{H}, \mathrm{Me}_{2} \mathrm{Si}\right) .{ }^{13} \mathrm{C}-\mathrm{NMR}: 145.9$ (s, 3 arom. C); $136.6\left(d, \mathrm{HC}=\mathrm{CH}_{2}\right)$; 130.1, 127.3, 126.0 (3d, 15 arom. C); $116.3\left(t, \mathrm{HC}=\mathrm{CH}_{2}\right) ; 83.1(d, \mathrm{SiCH}) ; 72.7(d,(\mathrm{OH}) \mathrm{C})$; $66.5\left(t, \mathrm{SiOCH}_{2}\right) ; 61.2(q, \mathrm{MeO}) ; 60.7\left(s, \mathrm{Ph}_{3} C\right) ;-1.1,-2.1\left(2 q, \mathrm{Me}_{2} \mathrm{Si}\right)$.

\section{(1'R)-1-[(1-Methoxy-2,2,2-triphenylethyl)(dimethyl)silyloxy]but-3-en-2-on (6)}<smiles>C=C[C@@H](O)CO[Si](C)(C)[C@@H](OC)C(c1ccccc1)(c1ccccc1)c1ccccc1</smiles>

G1

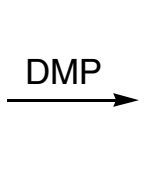<smiles>C=CC(=O)CO[Si](C)(C)[C@H](OC)C(c1ccccc1)(c1ccccc1)c1ccccc1</smiles>

DMP $(2.2 \mathrm{~mL}$ of a $15 \%$ soln. in $\mathrm{CH}_{2} \mathrm{Cl}_{2}, 1.02$ mmol) was added to a soln. of G1 (293.7 $\mathrm{mg}$,

$0.68 \mathrm{mmol})$ in $\mathrm{CH}_{2} \mathrm{Cl}_{2}(7.0 \mathrm{~mL})$ at $0{ }^{\circ} \mathrm{C}$. After $10 \mathrm{~min}$, the mixture was allowed to warm to 20 ${ }^{\circ} \mathrm{C}$ and stirred for $22 \mathrm{~h}$. Then, $\mathrm{MeOH}(0.3 \mathrm{~mL})$ was added. Evaporation of the combined organic fractions and column chromatography $\left(\mathrm{SiO}_{2}\right.$; hexane/EtOAc 20:1) afforded 6 (281.1 mg, $0.67 \mathrm{mmol}, 97 \%$ ) as a yellowish oil. IR: 2958s, 2924s, 1740s, 1700s, 1490s, 1445s, 1456s, 1250s, 1095s, 1080s, 830s. ${ }^{1} \mathrm{H}-\mathrm{NMR}: 7.31-7.14$ ( $m, 15$ arom. H); 6.50 ( $d d, J=10.3$, $\left.17.3,1 \mathrm{H}, H \mathrm{C}=\mathrm{CH}_{2}\right) ; 6.27(d d, J=2.0,17.3,1 \mathrm{H}, \mathrm{HC}=\mathrm{CHH}) ; 5.74(d d, J=2.0,10.3,1 \mathrm{H}$, $\mathrm{HC}=\mathrm{CH} H) ; 4.46(s, 1 \mathrm{H}, \mathrm{SiCH}) ; 4.16\left(s, \mathrm{SiOCH}_{2}\right) ; 3.34 \quad(s, 3 \mathrm{H}, \mathrm{MeO}) ;-0.11$, 
-0.32 (2s, 6H, Me $\mathrm{Si}_{2}{ }^{13} \mathrm{C}-\mathrm{NMR}: 198.3(s, \mathrm{CO}) ; 146.0$ (s, 3 arom. C); $131.9\left(d, \mathrm{HC}=\mathrm{CH}_{2}\right)$; 130.1, 127.4, 126.0 (3d, 15 arom. C); $128.6\left(t, \mathrm{HC}=C_{2}\right) ; 83.7(d, \mathrm{SiCH}) ; 67.9\left(t, \mathrm{SiOCH}_{2}\right)$; $61.1(q, \mathrm{MeO}) ; 60.7\left(s, \mathrm{Ph}_{3} C\right) ;-1.3,-1.6\left(2 q, \mathrm{Me}_{2} \mathrm{Si}\right) . \quad[\alpha]_{D}^{25}(\mathbf{6})=-71.8\left(\mathrm{c}=1.00, \mathrm{CHCl}_{3}\right)$. ESI-MS: $453\left([M+\mathrm{Na}]^{+}\right)$.

\section{2. [4+2] cycloaddition reactions}

\section{General procedure for the Diels-Alder reactions:}<smiles>[R]C1C2C=CC(C2)[C@H]1C(=O)CO[Si](C)(C)[C@H](OC)C(c1ccccc1)(c1ccccc1)c1ccccc1</smiles>

20a $(\mathrm{R}=\mathrm{H})$

21a $(R=M e)$<smiles>[R]C=CC(=O)CO[Si](C)(C)[C@H](OC)C(c1ccccc1)(c1ccccc1)c1ccccc1</smiles><smiles>CO[C@H](C(c1ccccc1)c1ccccc1)[Si](C)(OCC(=O)[C@H]1[C@H]2C=C[C@@H](C2)[C@H]1P)c1ccccc1</smiles>

A $1.0 \mathrm{M}$ soln. of $\mathrm{Mg}(\mathrm{OTf})_{2}$ in $\mathrm{Et}_{2} \mathrm{O}(1.4 \mathrm{~mL})$ was added to a solution of $\mathbf{6}$ or 7 (0.46 mmol) in $\mathrm{CH}_{2} \mathrm{Cl}_{2}(7.0 \mathrm{~mL})$. The mixture was cooled to $-78{ }^{\circ} \mathrm{C}$, freshly distilled dicyclopentadiene $(0.57$ $\mathrm{mL}, 7.0 \mathrm{mmol}$ ) added, and then stirring prolonged for $20 \mathrm{~min}$. After a filtration on a plough of $\mathrm{SiO}_{2}$, and evaporation of the solvent under reduced pressure, the crude mixture was purified on column chromatography ( $\mathrm{SiO}_{2}$; hexane/EtOAc 25:1). Diastereoselectivities were established by integration of the signals correspondent to the $\mathrm{SiMe}_{2}$ of the two diastereoisomers' absorptions in ${ }^{1} \mathrm{H}-\mathrm{NMR}$. Analytical data refer to the major isomer. 
$(1 ' R, 1 S, 2 S, 4 S)$ - and (1'R,1R,2R,4R)-1-(Bicyclo[2.2.1]hept-5-en-2-yl)-2-[(1-methoxy-2,2,2triphenylethyl)dimethylsilyloxy]ethanone (20a and 20b)

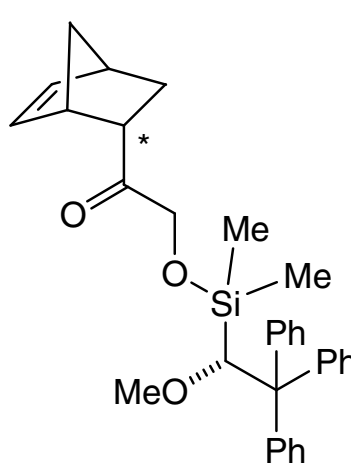

$20 a$ and $20 b$

This compound was prepared from 6 (200.0 $\mathrm{mg}, 0.46 \mathrm{mmol})$, according to the general procedure. After purification, a mixture of 20a and 20b (180.7 mg, $0.36 \mathrm{mmol}, 91 \%, d s>99 \%$ ) was obtained. IR: 3060s, 2970s, 2930s, 1720s, 1710s, 1490s, 1445s, 1436s, 1250s, 1092s, 1080s, 830s. ${ }^{1} \mathrm{H}-\mathrm{NMR}: 7.31-7.14$ (m, 15 arom. H); 6.17-6.14, 5.79-5.76 $(2 m, 2 \mathrm{H}, \mathrm{HC}=\mathrm{CH}) ; 4.46(s, 1 \mathrm{H}, \mathrm{SiCH}) ; 4.03,3.96(A B$, $\left.J=17.4,2 \mathrm{H}, \mathrm{SiOCH}_{2}\right) ; 3.35(s, 3 \mathrm{H}, \mathrm{MeO}) ; 3.16,2.90$ (2br.s. $2 \mathrm{H}$, allylic); 3.10-3.04 ( $m, 1 \mathrm{H}, \mathrm{COCH}) ; 1.77-1.22\left(m, 4 \mathrm{H}, 2 \mathrm{CH}_{2}\right) ;-0.09$, -0.35 (2s, 6H, Me $2 \mathrm{Si}) .{ }^{13} \mathrm{C}-\mathrm{NMR}: 209.7$ (s, CO); 146.0 ( $s$, arom. C); 137.8, 131.3 (2d, $\mathrm{HC}=\mathrm{CH}) ; 130.1,127.4,126.0$ (3d, arom. C); $83.7(d, \mathrm{SiCH}) ; 68.6\left(t, \mathrm{SiOCH}_{2}\right) ; 61.1(q$, $\mathrm{MeO}) ; 60.7\left(s, \mathrm{Ph}_{3} C\right) ; 50.0\left(t, \mathrm{CH}_{2}\right) ; 47.3,45.9,42.6(3 d, 3 \mathrm{CH}) ; 27.5\left(t, \mathrm{CH}_{2}\right) ;-1.3,-1.6(2 q$, $\left.\mathrm{Me}_{2} \mathrm{Si}\right)$. ESI-MS: $519\left([M+\mathrm{Na}]^{+}\right)$.

(1'R,1S,2S,3S,4S)- and (1'R,1R,2R,3R,4R)-1-(3-Methylbicyclo[2.2.1]hept-5-en-2-yl)-2-[(1methoxy-2,2,2-triphenylethyl)dimethylsilyloxy]ethanone (21a and 21b)

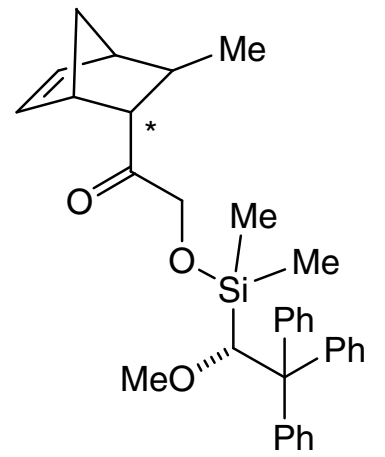

21a and 21b This compound was prepared from 7 (204.5 $\mathrm{mg}, 0.46 \mathrm{mmol})$, according to the general procedure. After purification, a mixture of $\mathbf{2 1 a}$ and 21b (204.4 mg, $0.40 \mathrm{mmol}, 87 \%$, $d s>99 \%$ ) was obtained. IR: $3058 s, 2964 s, 2931 s, 1717 s, 1708 s, 1490 s, 1442 s, 1439 s, 1247 s, 1090 s$, 1082s, 832s. ${ }^{1} \mathrm{H}-\mathrm{NMR}: 7.68-7.46$ (m, 15 arom. H); 6.60-6.53, 6.27$6.21(2 m, 2 \mathrm{H}, \mathrm{HC}=\mathrm{CH}) ; 4.79(s, 1 \mathrm{H}, \mathrm{SiCH}) ; 4.31,4.24(A B$, $\left.J=17.9,2 \mathrm{H}, \mathrm{SiOCH}_{2}\right) ; 3.67(s, 3 \mathrm{H}, \mathrm{MeO}) ; 3.16,2.90$ (2br.s. $2 \mathrm{H}$, allylic); 3.10-3.04 ( $m, 1 \mathrm{H}, \mathrm{COCH}) ; 1.41\left(d, J=6.5,2 \mathrm{H}, \mathrm{CH}_{3} \mathrm{CH}\right)$; 1.28-1.12 ( $\left.m, 4 \mathrm{H}, 2 \mathrm{CH}_{2}\right) ; 0.20,-0.01\left(2 s, 6 \mathrm{H}, \mathrm{Me}_{2} \mathrm{Si}\right) .{ }^{13} \mathrm{C}-\mathrm{NMR}: 210.4(s, \mathrm{CO}) ; 147.1(s$, arom. C); 138.1, $132.0(2 d, \mathrm{HC}=\mathrm{CH}) ; 130.6,127.7,126.9$ (3d, arom. C); 84.9 (d, SiCH); 68.9 $\left(t, \mathrm{SiOCH}_{2}\right) ; 61.5(q, \mathrm{MeO}) ; 60.8\left(s, \mathrm{Ph}_{3} C\right) ; 50.9\left(t, \mathrm{CH}_{2}\right) ; 47.6,46.4,42.9(3 d, 3 \mathrm{CH}) ; 27.9(t$, $\left.\mathrm{CH}_{2}\right) ; 26.4\left(q, \mathrm{CH}_{3}\right) ;-0.5,-1.1\left(2 q, \mathrm{Me}_{2} \mathrm{Si}\right)$. ESI-MS: $533\left([M+\mathrm{Na}]^{+}\right)$. 


\subsection{Stereochemical assignments}

\section{$(1 S, 2 S, 4 S)-1-(B i c y c l o[2.2 .1]$ hept-5-en-2-yl)-2-hydroxyethanone (H1)}

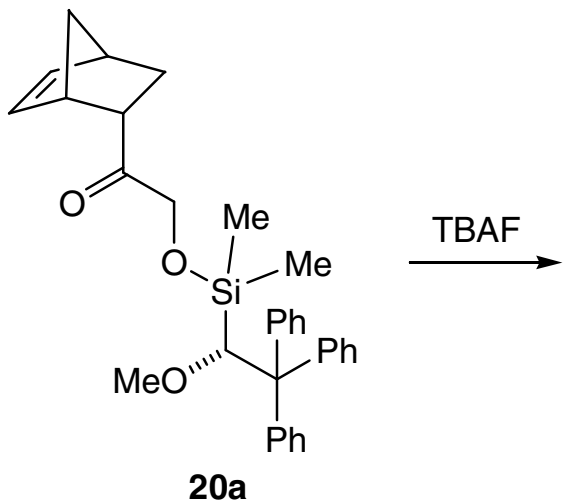

20a

A soln. of TBAF in THF $(1.0 \mathrm{M}, 0.2 \mathrm{ml}$, $0.20 \mathrm{mmol}$ ) was added to a soln. of $\mathbf{2 0 a}$ $(50.0 \mathrm{mg}, 0.10 \mathrm{mmol})$ in THF $(2.0 \mathrm{~mL})$ at $0{ }^{\circ} \mathrm{C}$. After $15 \mathrm{~min}$, a sat. aq. soln. of $\mathrm{NH}_{4} \mathrm{Cl}(2 \mathrm{~mL})$ was added. The two layers were separated and the aqueous phase was extracted with $\mathrm{Et}_{2} \mathrm{O}(2 \times 5 \mathrm{~mL})$. The combined organic layers were dried over $\mathrm{MgSO}_{4}$, and the solvent was removed under reduced pressure to afford the colorless oil $\mathbf{H 1}$ (14.9 $\mathrm{mg}, 0.097 \mathrm{mmol}$, 97\%), which was used in the following step without further purification. $[\alpha]_{D}^{25}(\mathbf{H 1})=-88.4\left(\mathrm{c}=1.40, \mathrm{CHCl}_{3}\right)$. IR: 3430s, 3060s, 1710s, 1570s, 1442s, $1459 s, 1247 s, 1090 s, 1080 s, 832 s .{ }^{1} \mathrm{H}-\mathrm{NMR}: 6.16(d d, 1 \mathrm{H}, J=3.1, J '=6.3, H \mathrm{C}=\mathrm{CHCHCH})$; $5.81\left(d d, 1 \mathrm{H}, J=3.1, J^{\prime}=6.3, \mathrm{HC}=\mathrm{CHCHCH}\right) ; 4.38,4.30\left(A B, J=16.5, \mathrm{OCH}_{2}\right) ; 3.22($ br.s, $\mathrm{OH})$; 3.40-2.65 ( $m, 3 \mathrm{H}$, allylic, $\mathrm{COCH}) ; 1.44-1.29\left(m, 2 \mathrm{CH}_{2}\right) \cdot{ }^{13} \mathrm{C}-\mathrm{NMR}: 210.4(s, \mathrm{CO})$; 138.1, $132.0(2 d, \mathrm{HC}=\mathrm{CH}) ; 68.9\left(t, \mathrm{OCH}_{2}\right) ; 50.9\left(t, \mathrm{CH}_{2}\right) ; 47.6,46.4,42.9(3 d, 3 \mathrm{CH}) ; 27.9(t$, $\left.\mathrm{CH}_{2}\right)$. ESI-MS: $175\left([M+\mathrm{Na}]^{+}\right)$.

\section{(1S,2S,4S)-Bicyclo[2.2.1]hept-5-ene-2-carboxylic acid (J1)}

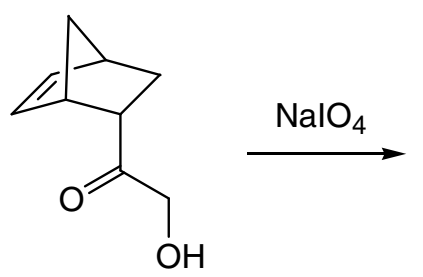

H1

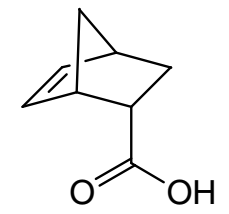

J1

$\mathrm{NaIO}_{4}(186.4 \mathrm{mg}, 0.66 \mathrm{mmol})$ was added to a soln. of $\mathbf{H 1}(14.6 \mathrm{mg}, 0.90 \mathrm{mmol})$ in a $1: 1$ mixture of $\mathrm{CH}_{3} \mathrm{CN} / \mathrm{H}_{2} \mathrm{O}(5.0 \mathrm{~mL})$, and it was stirred for $5 \mathrm{~h}$ at $23{ }^{\circ} \mathrm{C}$. The mixture was then extracted with $\mathrm{Et}_{2} \mathrm{O}$, an aq. soln. of $\mathrm{NaOH}(3.0 \mathrm{~mL}, 0.5 \mathrm{~m})$ was added, and the two phases separated. The $\mathrm{pH}$ of the aq. phase was then corrected to 6-7 by addition of an aq. soln. of $\mathrm{HCl}(0.740 \mathrm{~mL}, 10 \%), \mathrm{Et}_{2} \mathrm{O}$ was added to the mixture and the two phases were again separated. The final organic layer was dried over $\mathrm{MgSO}_{4}$ and filtered through a plough of silica gel to afford $\mathbf{J 1}(6.1 \mathrm{mg}, 0.04 \mathrm{mmol}, 67 \%)$ as a colorless solid. M.p. 33-34 ${ }^{\circ} \mathrm{C}$ (from oil). $[\alpha]_{D}^{25}=-126.3\left(\mathrm{c}=1.20, \mathrm{CHCl}_{3}\right)\left[(1 S, 2 S, 4 S)-\mathbf{J 1}:[\alpha]_{D}^{25}=-139.0(\mathrm{c}=1.38, \quad \mathrm{EtOH}){ }^{6}\right.$

\footnotetext{
${ }^{6}$ W. Choy, L.A. Reed, S. Masamune, J. Org. Chem. 1983, 48, 1137.
} 
$(1 R, 2 R, 4 R)-\mathbf{J 1}:[\alpha]_{D}^{25}=+140.0(\mathrm{c}=1.0 \mathrm{EtOH})^{7}$ ], confirming the configuration of $20 \mathbf{a}$ as shown above. IR: 2986s, 1708s, 1570s, 1444s, 1460s, 1250s, 1090s, 1082s, 830s. ${ }^{1} \mathrm{H}$ NMR: 10.2 (br.s, $\mathrm{COOH}) ; 6.13\left(d d, 1 \mathrm{H}, H \mathrm{C}=\mathrm{CHCHCH}, J=2.9, \mathrm{~J}^{\prime}=5.7\right) ; 5.90(d d, 1 \mathrm{H}$, $\left.\mathrm{HC}=\mathrm{CHCHCH}, J=2.9, J^{\prime}=5.5\right) ; 3.17$ (br.s, COCH); 3.01-2.90 ( $m, 2 \mathrm{H}$, allylic); 1.96-1.83 $\left(m, 1 \mathrm{H}, \mathrm{HCHCHCH}_{2} \mathrm{CHCO}\right), 1.44-1.21\left(\mathrm{~m}, 3 \mathrm{H}, \mathrm{HCHCHCH} \mathrm{CHCO}_{2} ;{ }^{13} \mathrm{C}-\mathrm{NMR}: 181.0(s\right.$, $\mathrm{COOH})$ 137.8, $132.3(2 d, \mathrm{HC}=\mathrm{CH}) ; 49.7\left(t, \mathrm{CH}_{2}\right) ; 45.7,43.3,42.6(3 d, 3 \mathrm{CH}) ; 29.1\left(t, \mathrm{CH}_{2}\right)$. CI-MS: $139\left(100,[M+\mathrm{H}]^{+}\right) ; 121\left(10,\left[\mathrm{M}-\mathrm{H}_{2} \mathrm{O}+\mathrm{H}\right]^{+}\right)$.

\section{Enantioselective synthesis of (-)-Frontalin}

\section{(R)-1-Methoxy-2,2,2-triphenylethyl)(dimethyl)silyloxyacetone $((R)-22)$}

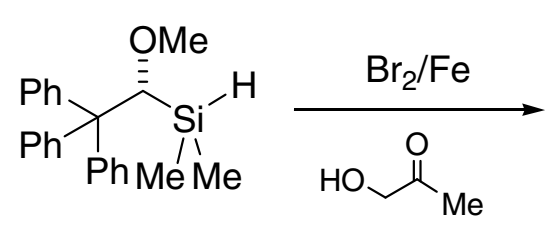

$(R)-3$<smiles>CO[C@@H](C(c1ccccc1)(c1ccccc1)c1ccccc1)[Si](C)(C)OCC(C)=O</smiles>

$(R)-22$ A soln. of $(R)-3(346.2 \mathrm{mg}, 1.00$ $\mathrm{mmol})$ in $\mathrm{CH}_{2} \mathrm{Cl}_{2}(15.0 \mathrm{~mL})$ was cooled to $-78{ }^{\circ} \mathrm{C}$, then $\mathrm{Br}_{2}(0.054$ $\mathrm{mL}, \quad 1.05 \mathrm{mmol})$ was added dropwise. The cooling bath was removed and the solvent evaporated at $23{ }^{\circ} \mathrm{C}$. The residual was dissolved in $\mathrm{CH}_{2} \mathrm{Cl}_{2}(20.0 \mathrm{~mL})$ and the mixture cooled to $0{ }^{\circ} \mathrm{C} \mathrm{NEt}_{3}(0.28 \mathrm{~mL}, 2.00$ mmol), hydroxyacetone (148.6 mg, $2.00 \mathrm{mmol})$ and DMAP (12.2 mg, $0.10 \mathrm{mmol})$ were added. The mixture was stirred at $23{ }^{\circ} \mathrm{C}$ for $1 \mathrm{~h}$, then quenched with $\mathrm{H}_{2} \mathrm{O}(10 \mathrm{~mL})$. The two layers were separated, and the aq. phase was extracted with $\mathrm{Et}_{2} \mathrm{O}(2 \times 20 \mathrm{~mL})$. The combined organic layers were dried over $\mathrm{MgSO}_{4}$ and the solvent was removed under reduced pressure. The crude mixture was purified by flash chromatography $\left(\mathrm{SiO}_{2}\right.$, hexane/EtO $\left.\mathrm{E}_{2} 10: 3\right)$ to afford $(R)-\mathbf{2 2}$ as a colorless oil $(401.8 \mathrm{mg}, 0.96 \mathrm{mmol}, 96 \%) .[\alpha]_{D}^{25}((R)-\mathbf{2 2})=-64.3 \quad(\mathrm{c}=1.10$, $\mathrm{CHCl}_{3}$ ). IR: 3050s, 2920s, 2890s, 2820s, 2140s, 1730s, 1720s, 1490s, 1445s, 1250s, 1150s, 1095s, 1080s, 835s. ${ }^{1} \mathrm{H}-\mathrm{NMR}: 7.31-7.14$ (m, 15 arom. H); $4.44(s, \mathrm{SiCH}) ; 3.94\left(s, \mathrm{SiOCH}_{2}\right)$; $3.33(s, \mathrm{MeO}) ; 2.06$ ( $s, \mathrm{MeCO}) ;-0.12,-0.33$ (2s, $\left.\mathrm{Me}_{2} \mathrm{Si}\right) .{ }^{13} \mathrm{C}-\mathrm{NMR}: 208.4$ ( $\left.s, \mathrm{MeCO}\right) ; 145.9$ (s, 3 arom. C); 130.1 (d, 3 arom. C); 127.4, 126.0 (2d, 2 x 6 arom. C); 83.7 (d, SiCH); 69.0 (t, $\left.\mathrm{SiOCH}_{2}\right) ; 61.1$ ( $\left.q, \mathrm{MeO}\right) ; 60.7\left(s, \mathrm{Ph}_{3} C\right) ; 25.9$ ( $\left.q, \mathrm{MeCO}\right) ;-1.4,-1.6\left(2 q, \mathrm{Me}_{2} \mathrm{Si}\right) . \mathrm{CI}-\mathrm{MS}: 436$ $\left(18,\left[M+\mathrm{NH}_{4}\right]^{+}\right) ; 131\left(100,\left[\mathrm{C}_{5} \mathrm{H}_{11} \mathrm{O}_{2} \mathrm{Si}\right]^{+}\right)$.

\footnotetext{
${ }^{7}$ C. Palomo, M. Oiarbide, J. M. García, A. González, A. Lecumberri, A. Linden, J. Am. Chem. Soc. 2002, 124, 10288
} 
$(1 ' R, 3 S)-\quad$ and $\quad(1 ' R, 3 R)-\quad 1-\{[1-M e t h o x y-2,2,2-t$ triphenylethyl](dimethyl)silyloxy $\}-2-$ methyl-5-(2-methyl-1,3-dioxolan-2-yl)-pentan-2-ol (24a and 24b)<smiles>CO[C@H](C(C)(c1ccccc1)c1ccccc1)[Si](C)(C)OCCC(C)=O</smiles>

$(R)-22$<smiles>CO[C@H](C(c1ccccc1)c1ccccc1)[Si](C)(C)OCC(C)(O)C1(C)OCCO1</smiles>

24a

A soln. of $\mathrm{MgBr}_{2}$ in $\mathrm{Et}_{2} \mathrm{O}(1.00 \mathrm{M}$, $0.40 \mathrm{~mL}, \quad 0.40$ mmol) was added to a soln. of $(R)-$

$22(180.1 \mathrm{mg}, 0.43 \mathrm{mmol})$ in $\mathrm{CH}_{2} \mathrm{Cl}_{2}(5.00 \mathrm{~mL})$. The mixture was cooled to $-78{ }^{\circ} \mathrm{C}$ and a soln. of 3-(2-Methyl-1,3-dioxolan-2-yl) prop-1-ylmagnesiumbromid ${ }^{8}$ in $\mathrm{Et}_{2} \mathrm{O}$ (2.0 M, $0.5 \mathrm{~mL}$, $1.00 \mathrm{mmol}$ ) was added dropwise. After $20 \mathrm{~min}$ the reaction was quenched with a sat. aq. soln. of $\mathrm{NH}_{4} \mathrm{Cl}(5 \mathrm{~mL})$. The two layers were separated, and the aq. phase was extracted with $\mathrm{Et}_{2} \mathrm{O}$ ( 2 x $20 \mathrm{~mL}$ ). The combined organic layers were dried over $\mathrm{MgSO}_{4}$ and the solvent was removed under reduced pressure. A mixture of $\mathbf{2 4 a}$ and $\mathbf{2 4 b}(45.5 \mathrm{mg}, 0.096 \mathrm{mmol}, 96 \%$, $\mathrm{dr}$ 90:1) was obtained as a colorless oil. $\left.[\alpha]_{D}^{25}=-51.1\left(\mathrm{c}=1.20, \mathrm{CHCl}_{3}\right)\right]$. IR: 3480br.m, 2958s, 2920s, 2860s, 1490s, 1445s, 1250s, 1100s, 1080s. ${ }^{1} \mathrm{H}-\mathrm{NMR}: 7.31-7.14$ (m, 15 arom. H); 4.41 $(s, 1 \mathrm{H}, \mathrm{SiCH}) ; 3.97-3.87\left(m, 4 \mathrm{H}, \mathrm{OCH}_{2} \mathrm{CH}_{2} \mathrm{O}\right) ; 3.32(s, 3 \mathrm{H}, \mathrm{MeO}) ; 3.2\left(s, 2 \mathrm{H}, \mathrm{SiOCH}_{2}\right) ; 1.85$ (br.s, 1H, OH); 1.62-1.24 (m, 6H, $\left.\left(\mathrm{CH}_{2}\right)_{3}\right) ; 1.31$ ( $\left.s, 3 \mathrm{H},(\mathrm{Me}) \mathrm{OCO}\right) ; 1.00(2 s, 3 \mathrm{H}, \mathrm{Me}(\mathrm{OH}) \mathrm{C})$; $-0.02,-0.44\left(2 s, 6 \mathrm{H}, \mathrm{Me}_{2} \mathrm{Si}\right) .{ }^{13} \mathrm{C}-\mathrm{NMR}: 145.9$ (s, 3 arom. C); 130.1, 127.3, 126.0 (3d, 15 arom. C); $110.0(s, \mathrm{OCO}) ; 83.1(d, \mathrm{SiCH}) ; 72.0(s,(\mathrm{OH}) \mathrm{C}) ; 69.7\left(t, \mathrm{SiOCH}_{2}\right) ; 64.6(t$, $\left.\mathrm{OCH}_{2} \mathrm{CH}_{2} \mathrm{O}\right) ; 61.1(q, \mathrm{MeO}) ; 60.7\left(s, \mathrm{Ph}_{3} C\right) ; 39.8,38.6,18.4\left(3 t,\left(\mathrm{CH}_{2}\right)_{3}\right) ; 23.7,23.0(2 q$, $(\mathrm{OH}) \mathrm{MeC}$ und $(\mathrm{Me}) \mathrm{OCO}) ;-1.1,-2.4\left(2 q, \mathrm{Me}_{2} \mathrm{Si}\right)$. ESI-MS: $571\left([\mathrm{M}+\mathrm{Na}]^{+}\right)$.

(S)-2-Methyl-5-(2-methyl-1,3-dioxolan-2-yl)pentan-1,2-diol (25)

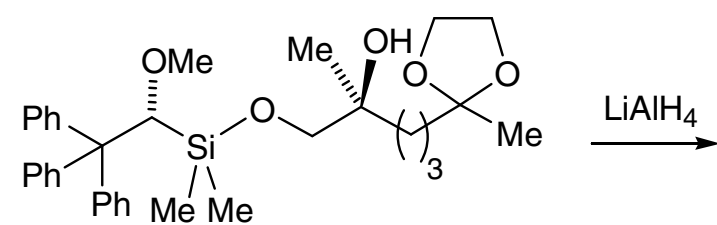

24a

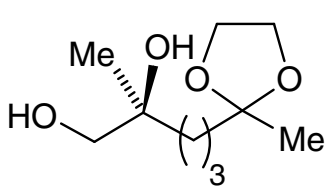

$(S)-25$

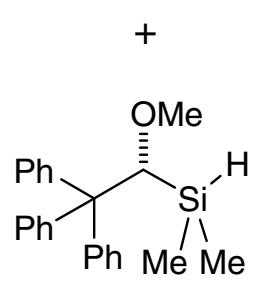

$(R)-3$

$\mathrm{LiAlH}_{4}(20.0 \mathrm{mg}, 0.15 \mathrm{mmol})$ was added to a soln. of $\mathbf{2 4 a}$ (80.0 mg, $0.15 \mathrm{mmol}$ ) in $\mathrm{Et}_{2} \mathrm{O}$ $(2.0 \mathrm{~mL})$ at $0{ }^{\circ} \mathrm{C}$. After 90 min, the solution was filtered (EtOAc) on $\mathrm{SiO}_{2}$ and the solvent removed under reduced pressure. Purification

\footnotetext{
${ }^{8}$ M. Ohwa, E. L. Eliel, Chem. Lett. 1987, 41.
} 
by flash chromatography ( $\mathrm{SiO}_{2}$; EtOAc) afforded $(R)$-MOTES-H $(R)-3$ (34.7 mg, 0.196 mmol, 95\%) and subsequently (S)-25 (28.1 $\mathrm{mg}, 0.14 \mathrm{mmol}, 95 \%)$ as a colorless oil. $[\alpha]_{D}^{25}(\mathbf{2 5})=-2.1\left(\mathrm{c}=1.06, \mathrm{CHCl}_{3}\right),[\alpha]_{D}^{25}(\mathbf{2 5})=-2.3\left(\mathrm{c}=1.05, \mathrm{Et}_{2} \mathrm{O}\right)$. Lit.: $\left.[\alpha]_{D}^{25}(S)-\mathbf{2 5}\right):$ $-1.8\left(\mathrm{c}=1.2, \mathrm{Et}_{2} \mathrm{O}\right)$, confirming the configuration of $24 \mathrm{a}$ as written above. ${ }^{1} \mathrm{H}-\mathrm{NMR}: 3.97-$ $3.87\left(m, 4 \mathrm{H}, \mathrm{OCH}_{2} \mathrm{CH}_{2} \mathrm{O}\right) ; 3.45,3.38\left(\mathrm{AB}, J=10.9,2 \mathrm{H},(\mathrm{OH}) \mathrm{CH}_{2}\right) ; 2.24$ (br.s, 2H, $\left.2 \mathrm{OH}\right)$; 1.66-1.62, 1.52-1.39 (2m, 6H, $\left.\left(\mathrm{CH}_{2}\right)_{3}\right) ; 1.30,1.15$ (2s, 6H, $\left.2 \mathrm{Me}\right) .{ }^{13} \mathrm{C}-\mathrm{NMR}: 110.0$ ( $\left.s, \mathrm{OCO}\right)$; $72.8(s,(\mathrm{OH}) \mathrm{C}) ; 69.7\left(t,(\mathrm{OH}) \mathrm{CH}_{2}\right) ; 64.5\left(t, \mathrm{OCH}_{2} \mathrm{CH}_{2} \mathrm{O}\right) ; 39.5,38.6,18.3\left(3 t,\left(\mathrm{CH}_{2}\right)_{3}\right) ; 23.7$, $23.1(2 q, \mathrm{Me}(\mathrm{OH}) \mathrm{C}$ und $(\mathrm{Me}) \mathrm{OCO})$.

\section{(-)-Frontalin}

\section{From $(R)-22$}<smiles>COC(=O)CO[Si](C)(C)[C@H](OC)C(c1ccccc1)(c1ccccc1)c1ccccc1</smiles>

$(R)-22$

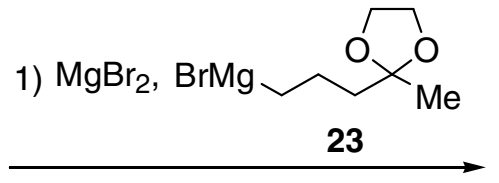

2) $\mathrm{HCl}, \mathrm{THF}$
(S)<smiles>CC12CCC(C)(O1)C(C)(C)OC2</smiles>

(-)-frontalin

A soln. of $\mathrm{MgBr}_{2}$ in $\mathrm{Et}_{2} \mathrm{O}(1.00 \mathrm{M}$, $4.0 \mathrm{~mL}, \quad 4.00$ mmol) was added to a soln. of $(R)-22(418.6 \mathrm{mg}, 1.00 \mathrm{mmol})$ in $\mathrm{CH}_{2} \mathrm{Cl}_{2}(10.0 \mathrm{~mL})$. The mixture was cooled to $-90{ }^{\circ} \mathrm{C}$, and a soln. of 3-(2-methyl-1,3-dioxolan-2-yl)propyl magnesiumbromide ${ }^{8}$ (23) in $\mathrm{CH}_{2} \mathrm{Cl}_{2}(1.0$ м, $3.0 \mathrm{~mL}, 3.00 \mathrm{mmol}$ ) was added dropwise. After $30 \mathrm{~min}$, THF (10 mL) and a cold aq. soln. of $\mathrm{HCl}(2.5 \mathrm{~mL}, 10 \%)$ were added, and it was stirred for $5 \mathrm{~h}$ at $23{ }^{\circ} \mathrm{C}$. Addition of a sat. aq. soln. of $\mathrm{NaHCO}_{3}(5 \mathrm{~mL})$, separation of the phases, evaporation of the organic fraction, and column chromatography $\left(\mathrm{SiO}_{2}\right.$, hexane/ $\mathrm{Et}_{2} \mathrm{O}$ 10:3) afforded (-)-frontalin ${ }^{9}$ (95.3 mg, 0.67 mmol, $67 \%, 98.5 \%$ ee) as a colorless oil.

\section{From 25:}

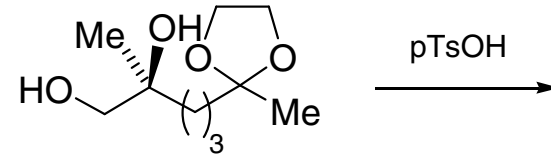

(S) -25

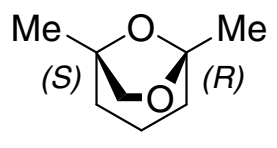

(-)-frontalin

Diol $(S)-25(10.0 \mathrm{mg}, 0.05 \mathrm{mmol})$ was dissolved in $\mathrm{CH}_{2} \mathrm{Cl}_{2}(1.0 \mathrm{~mL})$ and stirred at $23{ }^{\circ} \mathrm{C}$, then $\mathrm{pTsOH}(1.0 \mathrm{mg})$ was added and stirred prolonged for $2 \mathrm{~h}$. The reaction was filtered on $\mathrm{SiO}_{2}\left(\mathrm{Et}_{2} \mathrm{O}\right)$ and the solvent removed at reduced pressure. Purification by chromatography (hexane( $\left.\mathrm{Et}_{2} \mathrm{O} 10: 3\right)$ afforded (-)-frontalin ${ }^{9}(6.8 \mathrm{mg}, 0.05 \mathrm{mmol}, 98 \%, 99.2$ ee) as a colorless oil.

\footnotetext{
${ }^{9}$ J. S. Yadav, B. V. Joshi, A. B. Sahasrabudhe, Synth. Commun. 1985, 15, 797.
} 
$[\alpha]_{D}^{25}=-47.3\left(\mathrm{c}=1.60, \mathrm{Et}_{2} \mathrm{O}\right) . \mathrm{IR}: 2935 \mathrm{~s}, 2875 m, 1455 w, 1385 m, 1380 m, 1345 w, 1285 w$, $1260 m, 1240 m, 1205 m, 1175 m, 1120 s, 1065 m, 1030 s, 930 m, 895 m, 870 m, 820 m, 850 s$, 820m, 610w. ${ }^{1} \mathrm{H}-\mathrm{NMR}: 3.84(d, J=6.8, \mathrm{OCHH}) ; 3.46(d d, J=6.8,1.6, \mathrm{OCH}) ; 1.49-2.00$ $\left(m, \mathrm{CH}_{2} \mathrm{CH}_{2} \mathrm{CH}_{2}\right) ; 1.44$ (s, MeCOO); 1.33 (s, 3H, MeCH $\mathrm{CH}_{2} \mathrm{O} .{ }^{13} \mathrm{C}-\mathrm{NMR}: 108.1$ (s, COO); $80.1\left(s, \mathrm{CCH}_{2} \mathrm{O}\right) ; 74.2\left(t, \mathrm{CH}_{2} \mathrm{O}\right) ; 34.6,33.9\left(2 t, \mathrm{CH}_{2} \mathrm{CH}_{2} \mathrm{CH}_{2}\right) ; 24.7(q, \mathrm{MeCOO}) 23.1$ $\left(\mathrm{MeCH} \mathrm{C}_{2} \mathrm{O}\right) ; 18.0\left(\mathrm{CH}_{2} \mathrm{CH}_{2} \mathrm{CH}_{2}\right)$. EI-MS: $142\left(\mathrm{M}^{+\bullet}\right)$.

The enantiomeric ratio $(1 R, 5 S):(1 S, 5 R)=1: 260$ was established by GC (column: BGB Analytik AG; length $30 \mathrm{~m}$; stationary phase: BGB174; diameter: $0.25 \mu \mathrm{m}$; carrier gas $\mathrm{H}_{2}$ ): the column was warmed up to $50{ }^{\circ} \mathrm{C}$ for $5 \mathrm{~min}$, then the temperature increased of $3{ }^{\circ} \mathrm{C} \mathrm{min}{ }^{-1}$. Retention times: $\mathrm{t}_{(1 R, 5 S)}=7.25 \mathrm{~min}, \mathrm{t}_{(1 S, 5 R)}=7.51 \mathrm{~min}$.

\section{Enantioselective synthesis of (R)-1-Octane-1,3-diol}

$(1 ' S, 3 S)-$ and (1'S,3R)-1-[(1-Methoxy-2,2,2-triphenylethyl)dimethylsilyloxy]octan-3-ol (26a and 26b)<smiles>CO[C@@H](C(c1ccccc1)(c1ccccc1)c1ccccc1)[Si](C)(C)OCCC=O</smiles>

(S)-5<smiles>CC[C@@H](O)CCO[Si](C)(C)[C@H](OC)C(c1ccccc1)(c1ccccc1)c1ccccc1</smiles>

$26 a$ and $26 b$

A soln. of $\mathrm{MgBr}_{2}$ in $\mathrm{Et}_{2} \mathrm{O}$ $(1.00,0.40 \mathrm{~mL}$, $0.40 \mathrm{mmol})$ was added to a soln.

of $(S)-5$ (41.8 mg, $0.10 \mathrm{mmol})$ in $\mathrm{CH}_{2} \mathrm{Cl}_{2}(5.00 \mathrm{~mL})$. The mixture was cooled to $-78{ }^{\circ} \mathrm{C}$ and a soln. of Pentyl $\mathrm{MgBr}$ in $\mathrm{Et}_{2} \mathrm{O}(2.0 \mathrm{M}, 0.15 \mathrm{~mL}, 0.30 \mathrm{mmol})$ was added dropwise. After $20 \mathrm{~min}$ the reaction was quenched with a sat. aq. soln. of $\mathrm{NH}_{4} \mathrm{Cl}(5 \mathrm{~mL})$. The two layers were separated, and the aq. phase was extracted with $\mathrm{Et}_{2} \mathrm{O}(2 \times 20 \mathrm{~mL})$. The combined organic layers were dried over $\mathrm{MgSO}_{4}$ and the solvent was removed under reduced pressure. A mixture of 26a and 26b (45.5 mg, $0.096 \mathrm{mmol}, 96 \%$, dr 94:6) was obtained as a colorless oil. 26a was separated then from $\mathbf{2 6 b}$ by $\mathrm{SiO}_{2}$ chromatography on a preparative TLC (hexane/EtOAc 15:1, > 99\% de). IR: 3450br.m, 3062s, 2971s, 2926s, 2891s, 2823s, 1588s, 1491s, 1455s, 1253s, 1099s, 1084s. ${ }^{1} \mathrm{H}-\mathrm{NMR}: 7.49-7.40$ (m, 15 arom. H); 4.51 ( $\left.s, 1 \mathrm{H}, \mathrm{SiCH}\right)$; 4.02-3.89 ( $\left.m, 3 \mathrm{H}, \mathrm{SiOCH}_{2} \mathrm{CH}_{2} \mathrm{CH}\right)$; 3.50 ( $s, 3 \mathrm{H}, \mathrm{MeO}$ ); 3.11 (br.s, 1H, OH); 1.71-1.62 (m, $\left.2 \mathrm{H}, \mathrm{OCH}_{2} \mathrm{CH}_{2}\right) ; 1.62-1.40\left(m, 10 \mathrm{H}, \mathrm{OCH}_{2} \mathrm{CH}_{2}, \mathrm{Me}\left(\mathrm{CH}_{2}\right)_{4}\right) ; 1.12-1.03(t, 3 \mathrm{H}, J=6,2$, $\mathrm{MeCH}_{2}$ ); 0.00, -1.16 (2s, 6H, Me $\left.2 \mathrm{Si}\right) .{ }^{13} \mathrm{C}-\mathrm{NMR}: 146.8$ (s, 3 arom. C); 131.0 (d, 3 arom. C); 128.3 (d, 6 arom. C); 126.9 (d, 6 arom. C); $84.5(d, \mathrm{SiCH}) ; 72.3(d,(\mathrm{OH}) \mathrm{CH}) ; 62.6(t$, 
$\left.\mathrm{SiOCH}_{2}\right) ; 62.2$ (q, MeO); $61.6\left(s, \mathrm{Ph}_{3} C\right) ; 39.4,38.4,32.9,26.2,23.6\left(5 t, \mathrm{SiOCH}_{2} \mathrm{CH}_{2}\right.$, $\left.\mathrm{Me}\left(\mathrm{CH}_{2}\right)_{4}\right) ; 14.9\left(q, \mathrm{MeCH} \mathrm{CH}_{2}\right) ; 0.0,-1.0\left(2 q, \mathrm{Me}_{2} \mathrm{Si}\right)$. ESI-MS: $513\left([\mathrm{M}+\mathrm{Na}]^{+}\right)$.

(R)-1-Octane-1,3-diol (R)-(27)<smiles>CO[Si](C)(OCCC(O)[Al]=[W])C(c1ccccc1)C(c1ccccc1)(c1ccccc1)c1ccccc1</smiles><smiles>C[14CH](O)CCO</smiles>

(R)-27

A soln. of $\mathrm{LiAlH}_{4}$ in $\mathrm{Et}_{2} \mathrm{O}(1.0 \mathrm{M}$, $0.3 \mathrm{~mL}, 0.30 \mathrm{mmol})$ was added to a soln. of $26 \mathbf{a}(98.6 \mathrm{mg}, 0.20$ $\mathrm{mmol})$ in THF $(1.0 \mathrm{~mL})$ at $0{ }^{\circ} \mathrm{C}$. After $40 \mathrm{~min}$, a sat. aq. soln. of $\mathrm{NH}_{4} \mathrm{Cl}(2 \mathrm{~mL})$ was added, the<smiles>COC(C(c1ccccc1)(c1ccccc1)c1ccccc1)[Si](C)(C)C</smiles>

(S)-3 two layers were separated and the aq. phase was extracted with $\mathrm{Et}_{2} \mathrm{O}(2 \times 5 \mathrm{~mL})$. The combined organic layers were dried over $\mathrm{MgSO}_{4}$ and the solvent was removed under reduced pressure. Purification by flash chromatography $\left(\mathrm{SiO}_{2}\right.$; hexane/EtOAc 30:1 to 5:1) afforded $(R)$-MOTES-H $(R)-3(34.7 \mathrm{mg}$, $0.196 \mathrm{mmol}, 95 \%)$ and subsequently $(R)-27(29.8 \mathrm{mg}, 0.19 \mathrm{mmol}, 95 \%)$ as a colorless oil. $[\alpha]_{D}^{25}=-9.1\left(\mathrm{c}=1.50, \mathrm{CHCl}_{3}\right)\left[(R)-27:[\alpha]_{D}^{25}=-11.2\left(\mathrm{c}=1.32, \mathrm{CHCl}_{3}\right)\right]$, confirming the configuration as shown above. Analytical data in agreement with literature. ${ }^{10}$

\section{MOTES as a chiral derivatizing agent}

General procedure for the derivatization of alcohols and amines:

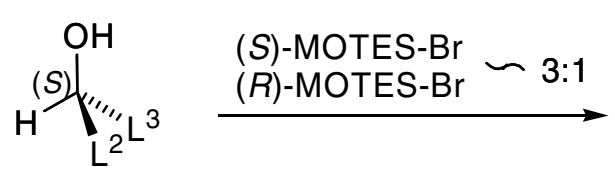

28-34

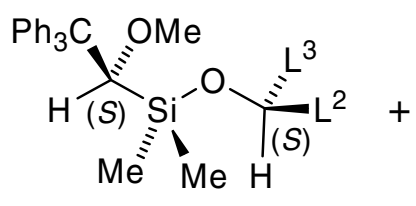

$35 a-41 a$<smiles>[3H]C([3H])([3H])O[Si](C)(C)[C@H](OC)c1ccccc1</smiles>

$35 b-41 b$

A soln. of $(S)-3(259.6 \mathrm{mg}, 0.75 \mathrm{mmol})$ and $(R)-3(86.5 \mathrm{mg}, 0.25 \mathrm{mmol})$ in $\mathrm{CH}_{2} \mathrm{Cl}_{2}(15.0 \mathrm{~mL})$ was cooled to $-78{ }^{\circ} \mathrm{C}$, then $\mathrm{Br}_{2}(0.054 \mathrm{~mL}, 1.05 \mathrm{mmol})$ was added dropwise. The cooling bath was removed and the solvent evaporated under reduced pressure. The residual was dissolved in $\mathrm{CH}_{2} \mathrm{Cl}_{2}(20.0 \mathrm{~mL})$, the mixture cooled to $0{ }^{\circ} \mathrm{C}$, and $\mathrm{NEt}_{3}(0.28 \mathrm{~mL}, 2.0 \mathrm{mmol})$, the desired alcohol 28-33 or amine 34 (2.0 mmol), and DMAP (12.2 mg, $0.01 \mathrm{mmol})$ were added. The mixture was stirred at $23{ }^{\circ} \mathrm{C}$ for $1 \mathrm{~h}$, quenched with $\mathrm{H}_{2} \mathrm{O}(10 \mathrm{~mL})$, and the two layers were

\footnotetext{
${ }^{10}$ T. Bauerle, P. Schreier, P. Brunerie, C. Bicchi, W. Schwab, Phytochemistry 1996, 43, 145.
} 
separated. The aq. phase was extracted with $\mathrm{Et}_{2} \mathrm{O}(2 \times 20 \mathrm{~mL})$, the combined organic layers were dried over $\mathrm{MgSO}_{4}$, and the solvent removed under reduced pressure.

All the crude mixtures were analyzed by NMR spectroscopy, and then purified by flash chromatography $\left(\mathrm{SiO}_{2}\right.$; hexane/EtOAc 10:1). Signals of each isomer are assigned from analysis of ${ }^{1} \mathrm{H}-\mathrm{NMR}$ spectra of the mixtures.

$(1 ' S, 2 S)$ - and (1'R,2S)-2-Butoxy-(1-methoxy-2,2,2-triphenylethyl)dimethylsilane (35a and 35b)<smiles>CC[C@H](C)O[Si](C)(C)[C@H](OC)C(c1ccccc1)(c1ccccc1)c1ccccc1</smiles>

$35 a$

$+$<smiles>CO[C@H](C(c1ccccc1)(c1ccccc1)c1ccccc1)[Si](C)(OC)OC(C)C[14CH3]</smiles>

35b

(S)-1-Butanol (28) (25.2 $\mathrm{mg}, 0.34 \mathrm{mmol})$ was derivatized according to general procedure. After chromatography a mixture of 35a and 35b (142.2 $\mathrm{mg}, 0.33 \mathrm{mmol}, 98 \%)$ was obtained as a colorless oil.

35a/35b: IR: 2980s, 2920s, 2880s, 2820s, 1490s, 1445s, 1250s, 1095s, 1080s. ESI-MS: $441\left([M+\mathrm{Na}]^{+}\right)$.

35a: ${ }^{1} \mathrm{H}-\mathrm{NMR}: 7.48-7.29$ ( $m, 15$ arom. H); 4.51 ( $\left.s, 1 \mathrm{H}, \mathrm{SiCH}\right)$; 3.89-3.68 (m, 1H, SiOCH); $3.49(s, 3 \mathrm{H}, \mathrm{MeO}) ; 1.62-1.51(m, 2 \mathrm{H}$, $\left.\mathrm{CH}_{2} \mathrm{CH}_{3}\right) ; 1.30-1.22\left(d, J=1.9, \mathrm{CH}_{3} \mathrm{CH}\right) ; 1.04-0.98(t, J=3.8$, $\left.\mathrm{CH}_{2} \mathrm{CH}_{3}\right) ; 0.00,-0.32\left(2 s, 6 \mathrm{H}, \mathrm{Me}_{2} \mathrm{Si}\right) .{ }^{13} \mathrm{C}-\mathrm{NMR}: 146.5(s, 3$ arom. C); 130.3 (d, 3 arom. C); 127.7 (d, 6 arom. C); 126.1 (d, 6 arom. C); 84.2 (d, SiCH); 70.0 (d, SiOCH); $61.0\left(s, \mathrm{Ph}_{3} C\right) ; 60.9$ (q, $\left.\mathrm{MeO}\right) ; 32.4\left(t, \mathrm{CH}_{2}\right) ; 23.3\left(q, \mathrm{CHCH}_{3}\right) ; 10.2$ (q, $\left.\mathrm{CH}_{2} \mathrm{CH}_{3}\right) ; 0.0,-1.0\left(2 q, \mathrm{Me}_{2} \mathrm{Si}\right)$.

35b: ${ }^{1} \mathrm{H}-\mathrm{NMR}: 7.48-7.29$ ( $m, 15$ arom. $\left.\mathrm{H}\right)$; 4.53 ( $\left.s, 1 \mathrm{H}, \mathrm{SiCH}\right) ; 3.89-3.68$ ( $\left.m, 1 \mathrm{H}, \mathrm{SiOCH}\right)$; $3.50(s, 3 \mathrm{H}, \mathrm{MeO}) ; 1.62-1.51\left(m, 2 \mathrm{H}, \mathrm{CH}_{2} \mathrm{CH}_{3}\right) ; 1.31-1.23\left(d, J=1.9, \mathrm{CH}_{3} \mathrm{CH}\right) ; 1.06-0.99(t$, $\left.J=3.8, \mathrm{CH}_{2} \mathrm{CH}_{3}\right) ; 0.00,-0.33\left(2 s, 6 \mathrm{H}, \mathrm{Me}_{2} \mathrm{Si}\right) .{ }^{13} \mathrm{C}-\mathrm{NMR}: 146.5$ (s, 3 arom. C); 130.3 (d, 3 arom. C); 127.7 (d, 6 arom. C); 126.1 (d, 6 arom. C); 84.3 (d, SiCH); 70.0 (d, SiOCH); 61.0 $\left(s, \mathrm{Ph}_{3} C\right) ; 60.9(q, \mathrm{MeO}) ; 32.5\left(t, \mathrm{CH}_{2}\right) ; 23.5\left(q, \mathrm{CHCH}_{3}\right) ; 10.3\left(q, \mathrm{CH}_{2} \mathrm{CH}_{3}\right) ; 0.1,-1.2(2 q$, $\mathrm{Me}_{2} \mathrm{Si}$ ). 
$(1 ' S, 2 S)-\quad$ and

(1’R,2S)-(1,2-Dimethylpropoxy)-(1-methoxy-2,2,2-triphenylethyl) dimethylsilane (36a and 36b)<smiles>CO[C@@H](C(c1ccccc1)(c1ccccc1)c1ccccc1)[Si](C)(C)O[C@H](C)C(C)C</smiles>

$36 a$<smiles>CO[C@@H](C(c1ccccc1)(c1ccccc1)c1ccccc1)[Si](C)(C)O[C@H](C)C(C)C</smiles>

$36 b$
(S)-3-Methylbutan-2-ol (29) (29.9 $\mathrm{mg}, \quad 0.34 \quad \mathrm{mmol})$ was derivatized according to general procedure. After chromatography a mixture of $\mathbf{3 6 a}$ and $\mathbf{3 6} \mathbf{b}(140.5 \mathrm{mg}, 0.31 \mathrm{mmol}$, $98 \%$ ) was obtained as a colorless oil.

36a/36b: IR: 2958s, 2920s, 2862s, 1490s, 1448s, 1250s, 1090s, 1085s. ESI-MS: $455\left([M+\mathrm{Na}]^{+}\right)$.

36a: ${ }^{1} \mathrm{H}-\mathrm{NMR}$ : 7.45-7.27 ( $m, 15$ arom. $\left.\mathrm{H}\right) ; 4.49$ ( $\left.s, 1 \mathrm{H}, \mathrm{SiCH}\right)$; 3.74-3.63 ( $m, 1 \mathrm{H}, \mathrm{SiOCH}) ; 3.47(s, 3 \mathrm{H}, \mathrm{MeO}) ; 1.79-1.67(m$, $1 \mathrm{H}, \mathrm{OCHCH}) ; 1.20-1.12\left(d, J=4.19, \mathrm{CH}_{3} \mathrm{CH}\right) ; 1.04-0.98(m$, $\left.6 \mathrm{H}, \mathrm{CHMe} e_{2}\right) ; 0.00,-0.33\left(2 s, 6 \mathrm{H}, \mathrm{Me}_{2} \mathrm{Si}\right) .{ }^{13} \mathrm{C}-\mathrm{NMR}: 146.4(s, 3$ arom. C); 130.4 (d, 3 arom. C); 127.9 (d, 6 arom. C); 126.3 (d, 6 arom. C); 84.3 (d, SiCH); $73.2(d, \mathrm{SiOCH}) ; 61.7\left(s, \mathrm{Ph}_{3} C\right) ; 61.1(q, \mathrm{MeO}) ; 35.5(t, \mathrm{OCHCH}) ; 20.3\left(q, \mathrm{CHCH}_{3}\right) ; 18.4(q$, $\left.\mathrm{CHMe} e_{2}\right) ; 0.0,-0.5\left(2 q, \mathrm{Me}_{2} \mathrm{Si}\right)$.

36b: ${ }^{1} \mathrm{H}-\mathrm{NMR}: 7.45-7.27$ ( $m, 15$ arom. $\left.\mathrm{H}\right)$; 4.51 ( $\left.s, 1 \mathrm{H}, \mathrm{SiCH}\right) ; 3.74-3.63$ ( $\left.m, 1 \mathrm{H}, \mathrm{SiOCH}\right)$; $3.48(s, 3 \mathrm{H}, \mathrm{MeO}) ; 1.79-1.67(m, 1 \mathrm{H}, \mathrm{OCHCH}) ; 1.22-1.14\left(d, J=4.19, \mathrm{CH}_{3} \mathrm{CH}\right) ; 1.06-1.00$ $\left(m, 6 \mathrm{H}, \mathrm{CHMe} e_{2}\right) ; 0.00,-0.34\left(2 s, 6 \mathrm{H}, \mathrm{Me}_{2} \mathrm{Si}\right) .{ }^{13} \mathrm{C}-\mathrm{NMR}: 146.4$ (s, 3 arom. C); $130.4(d, 3$ arom. C); 127.9 (d, 6 arom. C); 126.3 (d, 6 arom. C); 84.7 (d, SiCH); 73.5 (d, SiOCH); 61.7 $\left(s, \mathrm{Ph}_{3} C\right) ; 61.2(q, \mathrm{MeO}) ; 35.5(t, \mathrm{OCHCH}) ; 20.5\left(q, \mathrm{CHCH}_{3}\right) ; 18.6\left(q, \mathrm{CHMe}_{2}\right) ; 0.2,-0.7(2 q$, $\left.\mathrm{Me}_{2} \mathrm{Si}\right)$.

$(1 ' S, 2 S)-\quad$ and $\quad(1 ' R, 2 S)-(1-M e t h o x y-2,2,2-t r i p h e n y l e t h y l) d i m e t h y l-(1-p h e n y l$ ethoxy)silane (37a and 37b)<smiles>CO[C@H](O[Si](C)(C)C(c1ccccc1)(c1ccccc1)c1ccccc1)c1ccccc1</smiles>

$(S)$-1-Phenylethanol (30) $(41.5 \mathrm{mg}, 0.34 \mathrm{mmol})$ was derivatized according to general procedure. After chromatography a mixture of 37a and 37b (149.1 mg, $0.32 \mathrm{mmol}, 98 \%)$ was obtained as a colorless oil.

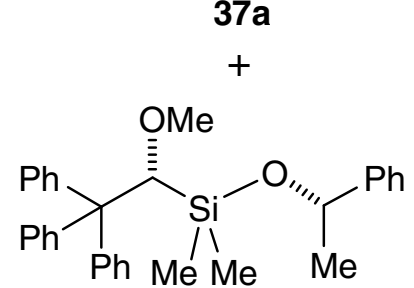

37a/37b: IR: 2955s, 2920s, 2865s, 2850s, 1490s, 1448s, 1250s, $1105 s, 1080 s$. ESI-MS: $489\left([M+\mathrm{Na}]^{+}\right)$.

37a: ${ }^{1} \mathrm{H}-\mathrm{NMR}: 7.58-7.31$ ( $m, 20$ arom. $\left.\mathrm{H}\right)$; 4.78-4.62 (m, 1H, $\mathrm{SiOCH}) ; 4.49(s, 1 \mathrm{H}, \mathrm{SiCH}) ; 3.31(s, 3 \mathrm{H}, \mathrm{MeO}) ; 1.53-1.41(d$, $\left.J=6.1, \mathrm{CHCH}_{3}\right) ;-0.29,-0.42\left(2 s, 6 \mathrm{H}, \mathrm{Me}_{2} \mathrm{Si}\right) .{ }^{13} \mathrm{C}-\mathrm{NMR}: 147.5(s$, 
3 arom. C); 145.6 (s, arom. C); 133.8, (d, 3 arom. C); 130.0 (d, 6 arom. C); 128.4, 127.9 (2d, 2 x 2 arom. C); 127.6 (d, 6 arom. C); 125.4 (d, arom. C); 83.4 (d, SiCH); 69.7 (d, SiOCH); 61.0 $\left(s, \mathrm{Ph}_{3} C\right) ; 62.3$ (q, $\left.\mathrm{MeO}\right) ; 25.7\left(q, \mathrm{CHCH}_{3}\right) ; 0.6,0.2\left(2 q, \mathrm{Me}_{2} \mathrm{Si}\right)$.

37b: ${ }^{1} \mathrm{H}-\mathrm{NMR}: 7.51-7.23$ ( $m, 20$ arom. H); 4.70-4.60 ( $\left.m, 1 \mathrm{H}, \mathrm{SiOCH}\right) ; 4.55(s, 1 \mathrm{H}, \mathrm{SiCH})$; $3.48(s, 3 \mathrm{H}, \mathrm{MeO}) ; 1.92-1.68\left(m, \mathrm{CH}_{2} \mathrm{CH}_{3}\right) ; 1.53-1.41\left(d, J=6.1, \mathrm{CHCH}_{3}\right) ;-0.00,-0.56(2 s$, 6H, $\mathrm{Me}_{2} \mathrm{Si}$ ). ${ }^{13} \mathrm{C}-\mathrm{NMR}: 147.8$ ( $s, 3$ arom. C); 146.1 (s, arom. C); 134.0, (d, 3 arom. C); 130.3 (d, 6 arom. C); 128.7, 127.7 (2d, 2 x 2 arom. C); 127.3 (d, 6 arom. C); 126.0 (d, arom. C); $85.8(d, \mathrm{SiCH}) ; 69.5(d, \mathrm{SiOCH}) ; 60.7\left(s, \mathrm{Ph}_{3} C\right) ; 62.4(q, \mathrm{MeO}) ; 25.9\left(q, \mathrm{CHCH}_{3}\right) ; 1.0,-0.1$ $\left(2 q, \mathrm{Me}_{2} \mathrm{Si}\right)$.

$(1 ' S, 2 S)-\quad$ and $\quad(1 ' R, 2 S)-\quad$ (1-Methoxy-2,2,2-triphenylethyl)dimethyl-(1-phenyl propoxy) silane (38a and $38 b)$<smiles>CC[C@H](O[Si](C)(C)C(OC)c1ccccc1)C(c1ccccc1)c1ccccc1</smiles>

$38 a$ $+$<smiles>CC[C@H](O[Si](C)(C)[C@H](OC)C(c1ccccc1)(c1ccccc1)c1ccccc1)c1ccccc1</smiles>

$38 b$

(S)-1-Phenylpropanol (31) (46.3 $\mathrm{mg}, 0.34 \mathrm{mmol}$ ) was derivatized according to general procedure. After chromatography a mixture of 38a and 38b (144.0 mg, $0.30 \mathrm{mmol}, 98 \%$ ) was obtained as a colorless oil.

38a/38b: IR: $3050 s, 2920 s, 2860 s, 2820 s, 1490 s, 1442 s, 1250 s$, 1115s, 1095s, 1080s, 935s. ESI-MS: $503\left([M+\mathrm{Na}]^{+}\right)$.

38a: ${ }^{1} \mathrm{H}-\mathrm{NMR}$ : 7.51-7.23 ( $m, 20$ arom. $\left.\mathrm{H}\right)$; 4.70-4.60 (m, $1 \mathrm{H}$, $\mathrm{SiOCH}) ; 4.52(s, 1 \mathrm{H}, \mathrm{SiCH}) ; 3.40(s, 3 \mathrm{H}, \mathrm{MeO}) ; 1.92-1.68(\mathrm{~m}$, $\left.\mathrm{CH}_{2}\right) ; 1.04-0.93\left(t, 3 \mathrm{H}, J=7.2, \mathrm{CH}_{2} \mathrm{CH}_{3}\right) ;-0.20,-0.34(2 s, 6 \mathrm{H}$, $\mathrm{Me}_{2} \mathrm{Si}$ ). ${ }^{13} \mathrm{C}-\mathrm{NMR}: 147.8$ ( $s, 3$ arom. C); 146.1 ( $s$, arom. C); 134.0, (d, 3 arom. C); 130.3 (d, 6 arom. C); 128.7, 127.7 (2d, 2 x 2 arom. C); 127.3 (d, 6 arom. C); 126.0 (d, arom. C); 83.7 (d, SiCH); 69.5 (d, $\mathrm{SiOCH}) ; 60.7$ (s, $\left.\mathrm{Ph}_{3} C\right) ; 62.2(q, \mathrm{MeO}) ; 34.9$ ( $t$, $\left.\mathrm{CH}_{2}\right) ; 11.4\left(q, \mathrm{CH}_{2} \mathrm{CH}_{3}\right) ; 0.9,0.4\left(2 q, \mathrm{Me}_{2} \mathrm{Si}\right)$.

38b: ${ }^{1} \mathrm{H}-\mathrm{NMR}: 7.51-7.23$ ( $m, 20$ arom. H); 4.70-4.60 ( $\left.m, 1 \mathrm{H}, \mathrm{SiOCH}\right) ; 4.58(s, 1 \mathrm{H}, \mathrm{SiCH})$; $3.53(s, 3 \mathrm{H}, \mathrm{MeO}) ; 1.92-1.68\left(m, \mathrm{CH}_{2}\right) ; 1.04-0.93\left(t, 3 \mathrm{H}, J=7.2, \mathrm{CH}_{2} \mathrm{CH}_{3}\right) ;-0.00,-0.56(2 s$, 6H, $\mathrm{Me}_{2} \mathrm{Si}$ ). ${ }^{13} \mathrm{C}$-NMR: 147.8 ( $s, 3$ arom. C); 146.1 ( $s$, arom. C); 134.0, (d, 3 arom. C); 130.3 (d, 6 arom. C); 128.7, 127.7 (2d, 2 × 2 arom. C); 127.3 (d, 6 arom. C); 126.0 (d, arom. C); $85.8(d, \mathrm{SiCH}) ; 69.5(d, \mathrm{SiOCH}) ; 60.7\left(s, \mathrm{Ph}_{3} C\right) ; 62.4(q, \mathrm{MeO}) ; 34.9\left(t, C \mathrm{H}_{2}\right) ; 11.6(q$, $\left.\mathrm{CH}_{2} \mathrm{CH}_{3}\right) ; 1.2,0.0\left(2 q, \mathrm{Me}_{2} \mathrm{Si}\right)$. 
$(1 ' S, 2 S)-\quad$ and

(1'R,2S)-(1-Methoxy-2,2,2-triphenylethyl)dimethyl-(1-naphth-1ylpropoxy)silane (39a and 39b)<smiles>COC(C(c1ccccc1)(c1ccccc1)c1ccccc1)[Si](C)(C)O[C@H](C)c1ccccc1</smiles>

$39 a$

$+$<smiles>CO[C@@H](C(c1ccccc1)(c1ccccc1)c1ccccc1)[Si](C)(C)O[C@H](C)c1ccccc1</smiles>

$39 \mathrm{~b}$

(S)-1-Naphth-1-ylethanol (32) $\quad(58.2 \quad \mathrm{mg}, \quad 0.34 \quad \mathrm{mmol})$ was derivatized according to general procedure. After chromatography a mixture of 39a and 39b (165.3 mg, $0.32 \mathrm{mmol}, 98 \%$ ) was obtained as a colorless oil.

39a/39b: IR: 3090s, 3050s, 2920s, 2860s, 2820s, 1490s, 1442s, $1250 s, 1115 s, 1095 s, 1080 s, 935 s$. ESI-MS: $539\left([M+\mathrm{Na}]^{+}\right)$.

39a: ${ }^{1} \mathrm{H}-\mathrm{NMR}:$ 8.33-8.25, 8.12-8.06, 8.00-7.92, 7.89-7.78 (4m, 4 x arom. H); 7.73-7.62 ( $m, 2 \mathrm{H}, 2$ x arom. $\mathrm{H}) ; 7.57-6.85$ ( $m, 16$ arom. $\mathrm{H})$; 5.76-5.68 (m, 1H, SiOCH); $4.68(s, 1 \mathrm{H}, \mathrm{SiCH}) ; 3.51(s, 3 \mathrm{H}$, $\mathrm{OMe}) ; 1.78-1.73(d, 3 \mathrm{H}, J=6.5, \mathrm{CHMe}) ; 0.00,-0.20(2 s, 6 \mathrm{H}$, $\mathrm{Me}_{2} \mathrm{Si}$ ). ${ }^{13} \mathrm{C}-\mathrm{NMR}: 145.6$ (s, 3 arom. C); 144.2 (s, arom. C); 134.2, 135.1 (2s, 2 arom. C); 129.9 (d, 3 arom. C); 129.6, 129.2, 129.8 (3d, 3 arom. C); 127.4 (d, 6 arom. C); 125.9 (d, 6 arom. C); 125.5, 124.6 (2d, 2 x 2 arom. C); 123.4, 123.5 (2d, 2 arom. C); 83.2 (d, SiCH); 68.8 $(d, \mathrm{SiOCH}) ; 61.1\left(s, \mathrm{Ph}_{3} C\right) ; 61.3(q, \mathrm{MeO}) ; 26.3\left(q, \mathrm{CHCH}_{3}\right) ;-0.2,-0.4\left(2 q, \mathrm{Me}_{2} \mathrm{Si}\right)$.

39b: ${ }^{1} \mathrm{H}-\mathrm{NMR}:$ 8.33-8.25, 8.12-8.06, 8.00-7.92, 7.89-7.78 (4m, 4 x arom. H); 7.73-7.62 (m, 2H, 2 x arom. H); 7.57-6.85 (m, 16 arom. H); 5.76-5.68 ( $m, 1 \mathrm{H}, \mathrm{SiOCH}) ; 4.72(s, 1 \mathrm{H}, \mathrm{SiCH})$; $3.64(s, 3 \mathrm{H}, \mathrm{OMe}) ; 1.82-1.75(d, 3 \mathrm{H}, J=6.5, \mathrm{CHMe}) ; 0.18,-0.38\left(2 s, 6 \mathrm{H}, \mathrm{Me}_{2} \mathrm{Si}\right) .{ }^{13} \mathrm{C}-\mathrm{NMR}$ : 145.9 (s, 3 arom. C); 144.2 ( $s$, arom. C); 134.2, 135.1 (2s, 2 arom. C); 129.9 (d, 3 arom. C); 129.6, 129.2, 129.8 (3d, 3 arom. C); 127.4 (d, 6 arom. C); 125.9 (d, 6 arom. C); 125.5, 124.6 (2d, 2 × 2 arom. C); 123.4, 123.5 (2d, 2 arom. C); 83.3 (d, SiCH); 68.9 (d, SiOCH); 61.1 ( $s$, $\left.\mathrm{Ph}_{3} C\right) ; 61.5$ ( $\left.q, \mathrm{MeO}\right) ; 26.4\left(q, \mathrm{CHCH}_{3}\right) ; 0.0,-0.7\left(2 q, \mathrm{Me}_{2} \mathrm{Si}\right)$. 
(1'S,2S)- $\quad$ and $\quad(1 ' R, 2 S)-2-[(1-M e t h o x y-2,2,2-t$ triphenylethyl)dimethylsilanyloxy] butyric acid methyl ester (40a and $40 \mathrm{~b})$<smiles>COC(=O)[C@H](C)O[Si](C)(C)[C@H](OC)C(c1ccccc1)(c1ccccc1)c1ccccc1</smiles>

$40 a$

$+$<smiles>COC(=O)[C@H](C)O[Si](C)(C)[C@H](OC)C(c1ccccc1)(c1ccccc1)c1ccccc1</smiles>

$40 \mathrm{~b}$

$(S)$-Methyl lactate $(33)(40.1 \mathrm{mg}, 0.34 \mathrm{mmol})$ was derivatized according to general procedure. After chromatography a mixture of $40 \mathrm{a}$ and $40 \mathrm{~b}(150.7 \mathrm{mg}, 0.31 \mathrm{mmol}, 98 \%)$ was obtained as a colorless oil.

40a/40b: IR: 2960br.s, 2860s, 2820s, 1770s, 1480s, 1442s, 1250s, 1115s, 1095s, 1080s, 935s. ESI-MS: $471\left([M+\mathrm{Na}]^{+}\right)$. 40a: ${ }^{1} \mathrm{H}-\mathrm{NMR}$ : 7.58-7.37 ( $m, 15$ arom. $\left.\mathrm{H}\right)$; 4.68 ( $\left.s, 1 \mathrm{H}, \mathrm{SiCH}\right)$; 4.41-4.32 (s, 1H, SiOCH); $3.91(s, 3 \mathrm{H}, \mathrm{COOMe}) ; 3.55(s, 3 \mathrm{H}$, CHOMe $) ; 1.55-1.51(d, 3 \mathrm{H}, J=6.9, \mathrm{CHMe}) ; 0.00,-0.12(2 s$, $6 \mathrm{H}, \mathrm{Me}_{2} \mathrm{Si}$ ). ${ }^{13} \mathrm{C}-\mathrm{NMR}: 146.3$ (s, 3 arom. C); 130.5 (d, 6 arom. C); 127.7 (d, 6 arom. C); 126.2 (d, 3 arom. C); 83.7 (d, SiCH); $68.3(d, \mathrm{SiOCH}) ; 61.3(s$, $\left.\mathrm{Ph}_{3} C\right) ; 61.0$ ( $\left.q, \mathrm{COOMe}\right) ; 52.1$ ( $\left.t, \mathrm{CHCOMe}\right) ; 21.4\left(q, \mathrm{CHCH}_{3}\right) ;-1.0,-1.1\left(2 q, \mathrm{Me}_{2} \mathrm{Si}\right)$.

40b: ${ }^{1} \mathrm{H}-\mathrm{NMR}: 7.58-7.37$ ( $m, 15$ arom. $\left.\mathrm{H}\right)$; 4.72 ( $\left.s, 1 \mathrm{H}, \mathrm{SiCH}\right)$; 4.50-4.42 ( $\left.s, 1 \mathrm{H}, \mathrm{SiOCH}\right)$; $3.91(s, 3 \mathrm{H}, \mathrm{COOMe}) ; 3.58(s, 3 \mathrm{H}, \mathrm{CHOMe}) ; 1.60-1.56(d, 3 \mathrm{H}, J=6.9, \mathrm{CH} M e) ; 0.16,-0.22$ (2s, 6H, $\mathrm{Me}_{2} \mathrm{Si}$ ). ${ }^{13} \mathrm{C}-\mathrm{NMR}: 146.3$ (s, 3 arom. C); 130.5 (d, 6 arom. C); 127.7 (d, 6 arom. C); 126.2 (d, 3 arom. C); $84.2(d, \mathrm{SiCH}) ; 68.3(d, \mathrm{SiOCH}) ; 61.3\left(s, \mathrm{Ph}_{3} C\right) ; 61.0(q, \mathrm{COOMe}) ; 52.1$ ( $t, \mathrm{CHCOMe}) ; 21.6\left(q, \mathrm{CHCH}_{3}\right) ; 0.0,-1.8\left(2 q, \mathrm{Me}_{2} \mathrm{Si}\right)$.

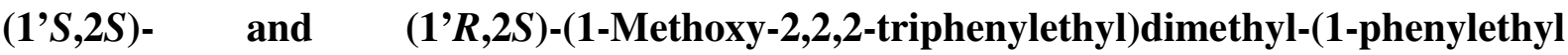
amino)silane (41a and 41b)<smiles>CO[C@@H]([C@H](N[C@H](C)c1ccccc1)c1ccccc1)[Si](C)(C)c1ccccc1</smiles>

$41 \mathrm{a}$ $+$<smiles>CO[C@@H](C(c1ccccc1)(c1ccccc1)c1ccccc1)[Si](C)(C)N[C@H](C)c1ccccc1</smiles>

41b

(S)-1-Phenylethylamine (34) (41.0 $\mathrm{mg}, 0.34 \mathrm{mmol})$ was derivatized according to general procedure. After chromatography a mixture of 41a and 41b (57.0 mg, $0.33 \mathrm{mmol}, 36 \%)$ was obtained as a colorless oil.

41a/41b: IR: 3090br.s, 2920s, 2860s, 2820s, 1600s, 1490s, 1442s, $1250 s, 1115 s, 1095 s, 1080 s, 1000 s$.

41a: ${ }^{1}$ H-NMR: $7.58-7.31$ ( $m, 20$ arom. $\left.\mathrm{H}\right) ; 4.49$ ( $\left.s, 1 \mathrm{H}, \mathrm{SiCH}\right) ; 4.00$ $3.89(m, 1 \mathrm{H}, \mathrm{SiOCH}) ; 3.31(s, 3 \mathrm{H}, \mathrm{MeO}) ; 1.53-1.41 \quad(d$, $J=5.9, \mathrm{CHCH}_{3}$ ); 1.38 (br. $\left.s, \mathrm{NH}\right) ; 0.00,-0.09$ (2s, 6H, $\mathrm{Me}_{2} \mathrm{Si}$ ).

${ }^{13}$ C-NMR: 147.5 ( $s, 3$ arom. C); 145.6 ( $s$, arom. C); 133.8, (d, 3 arom. C); 130.0 (d, 6 arom. C); 128.4, 127.9 (2d, 2 x 2 arom. C); 127.6 (d, 6 arom. C); 125.4 
(d, arom. C); $83.4(d, \mathrm{SiCH}) ; 69.7(d, \mathrm{SiOCH}) ; 61.0\left(s, \mathrm{Ph}_{3} C\right) ; 62.3(q, \mathrm{MeO}) ; 25.4(q$, $\left.\mathrm{CHCH}_{3}\right) ; 0.6,0.2\left(2 q, \mathrm{Me}_{2} \mathrm{Si}\right)$.

41b: ${ }^{1} \mathrm{H}-\mathrm{NMR}: 7.51-7.23$ ( $m, 20$ arom. H); 4.55 ( $\left.s, 1 \mathrm{H}, \mathrm{SiCH}\right) ; 4.00-3.89$ ( $\left.m, 1 \mathrm{H}, \mathrm{SiOCH}\right)$; $3.48(s, 3 \mathrm{H}, \mathrm{MeO}) ; 1.92-1.68\left(m, \mathrm{CH}_{2} \mathrm{CH}_{3}\right) ; 1.53-1.41\left(d, J=5.9, \mathrm{CHCH}_{3}\right) ; 1.38$ (br.s, NH); $0.10,-1.72\left(2 s, 6 \mathrm{H}, \mathrm{Me}_{2} \mathrm{Si}\right) .{ }^{13} \mathrm{C}-\mathrm{NMR}: 147.8$ ( $s, 3$ arom. C); 146.1 (s, arom. C); 134.0, (d, 3 arom. C); 130.3 (d, 6 arom. C); 128.7, 127.7 (2d, 2 x 2 arom. C); 127.3 (d, 6 arom. C); 126.0 (d, arom. C); 85.8 (d, SiCH); 69.5 (d, SiOCH); $60.7\left(s, \mathrm{Ph}_{3} C\right) ; 62.4$ (q, $\left.\mathrm{MeO}\right) ; 25.5$ (q, $\left.\mathrm{CHCH}_{3}\right) ; 1.0,-0.1\left(2 q, \mathrm{Me}_{2} \mathrm{Si}\right)$. 


\section{Copies of the spectra.}
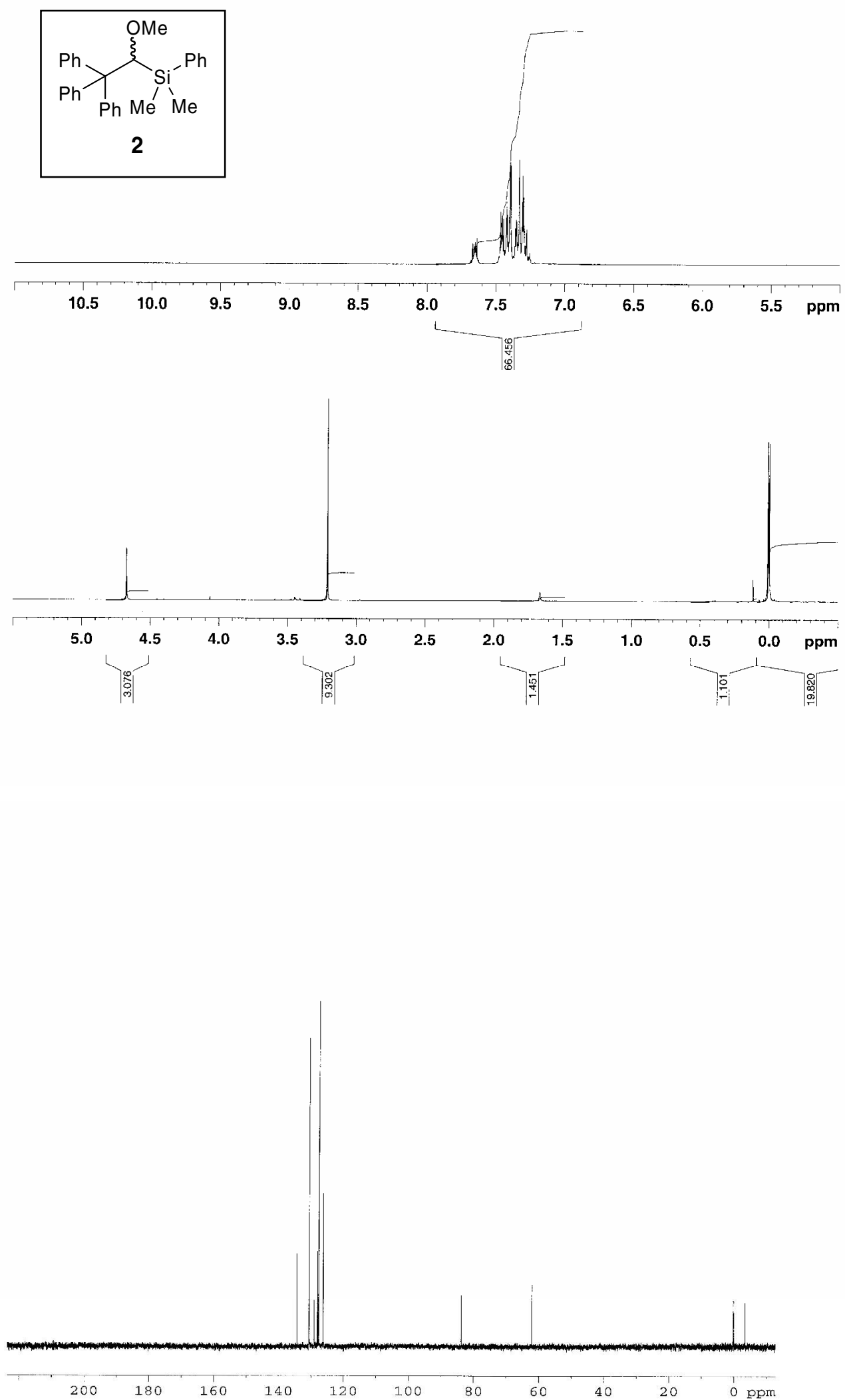

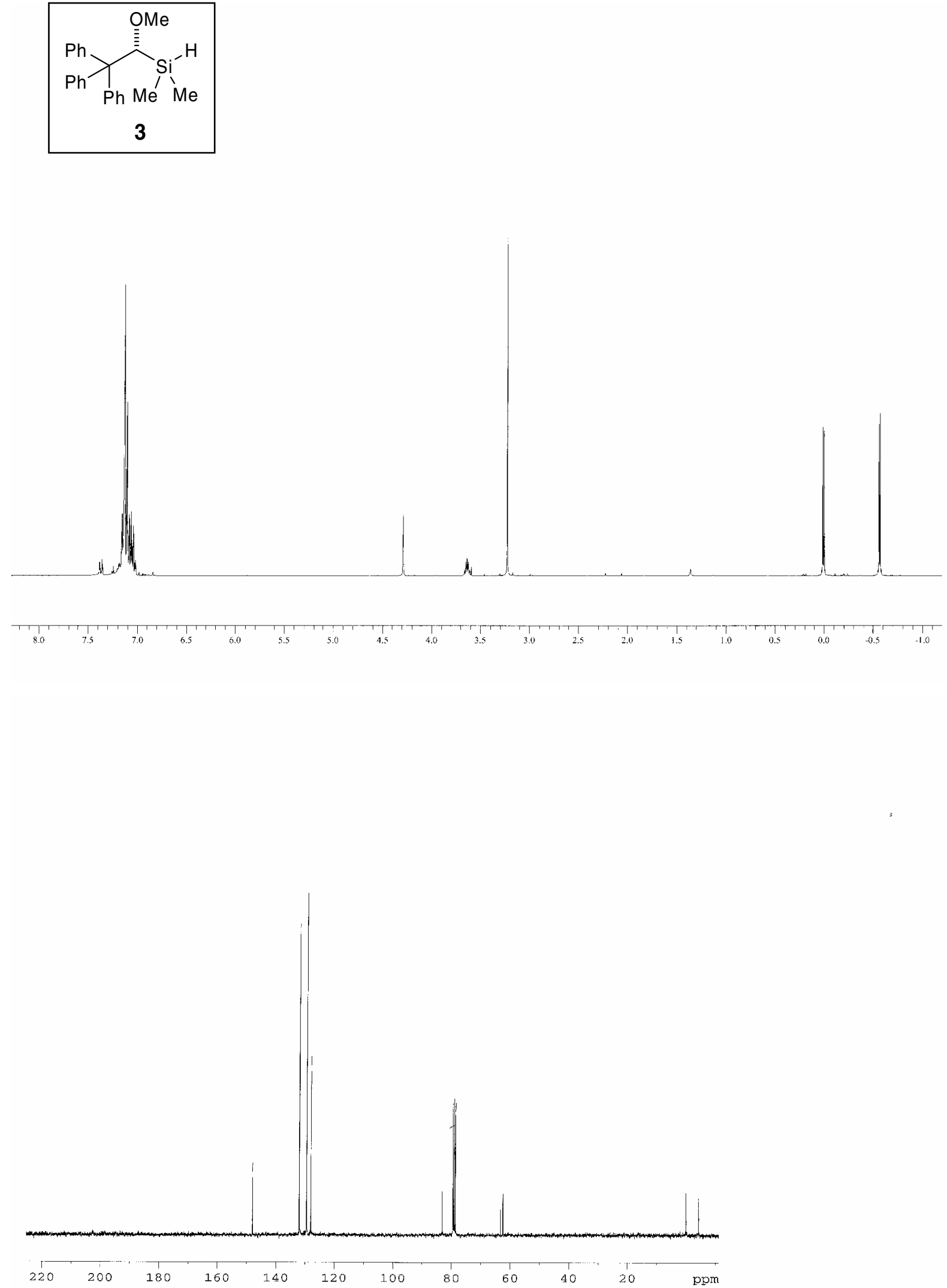

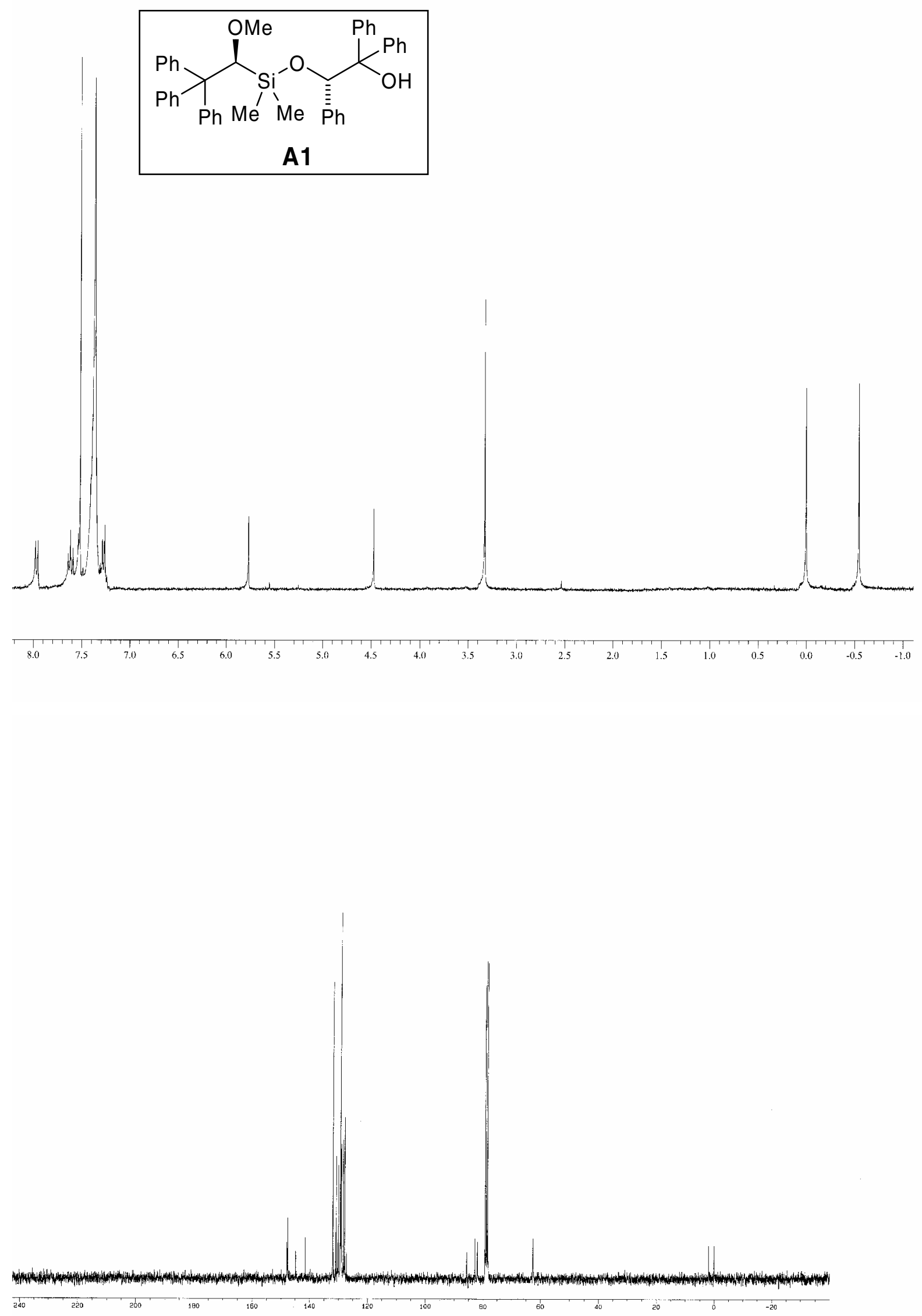

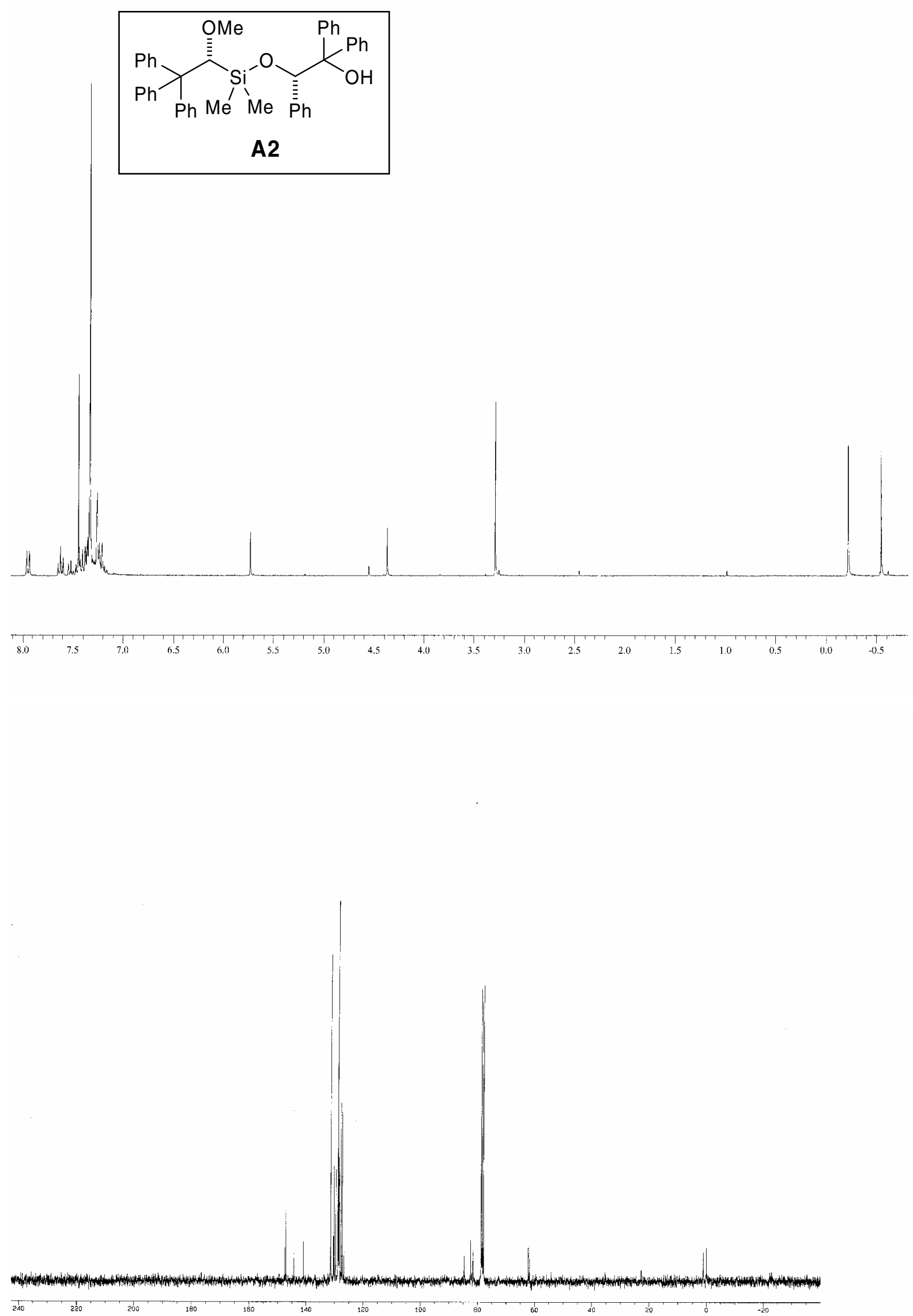

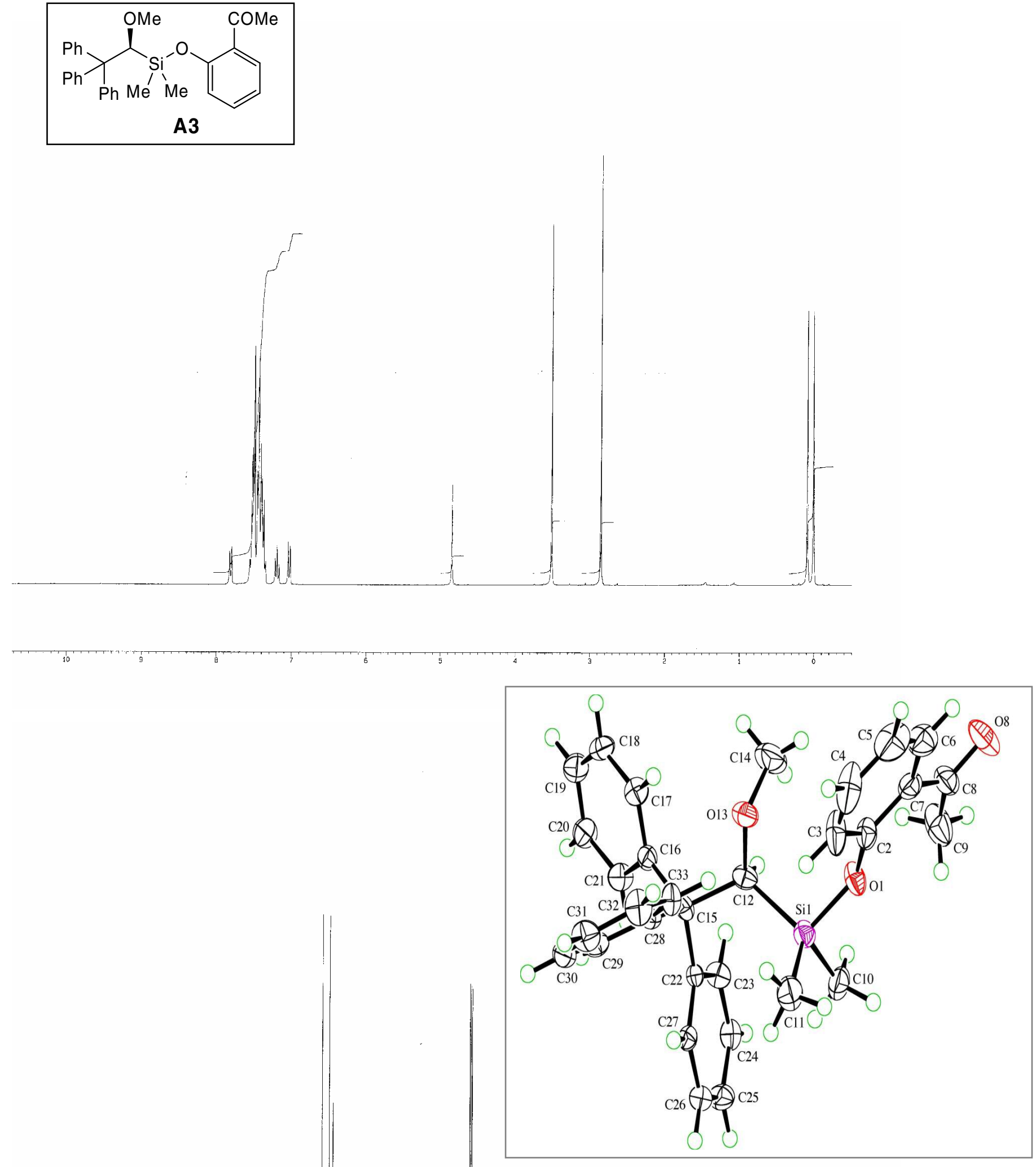

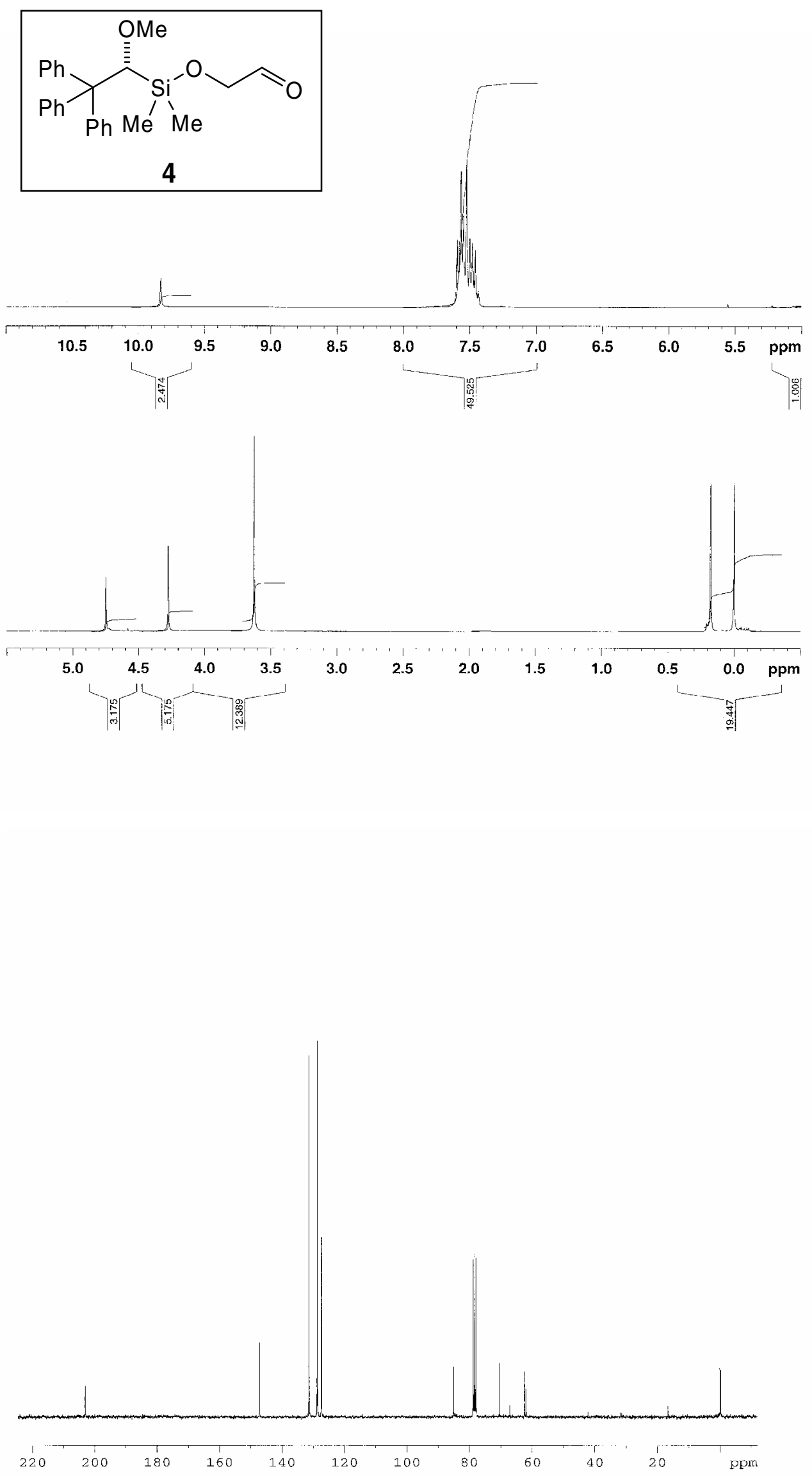

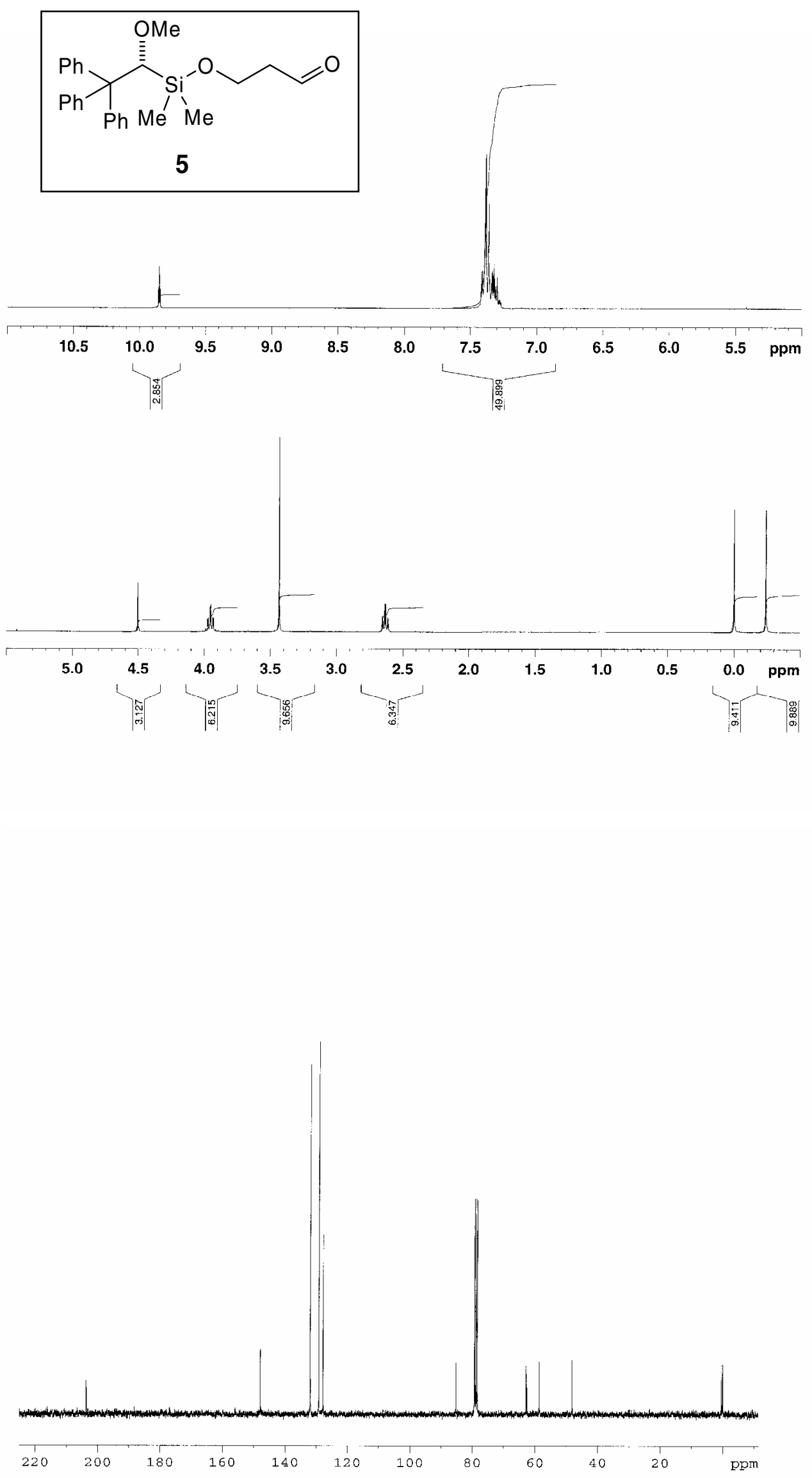

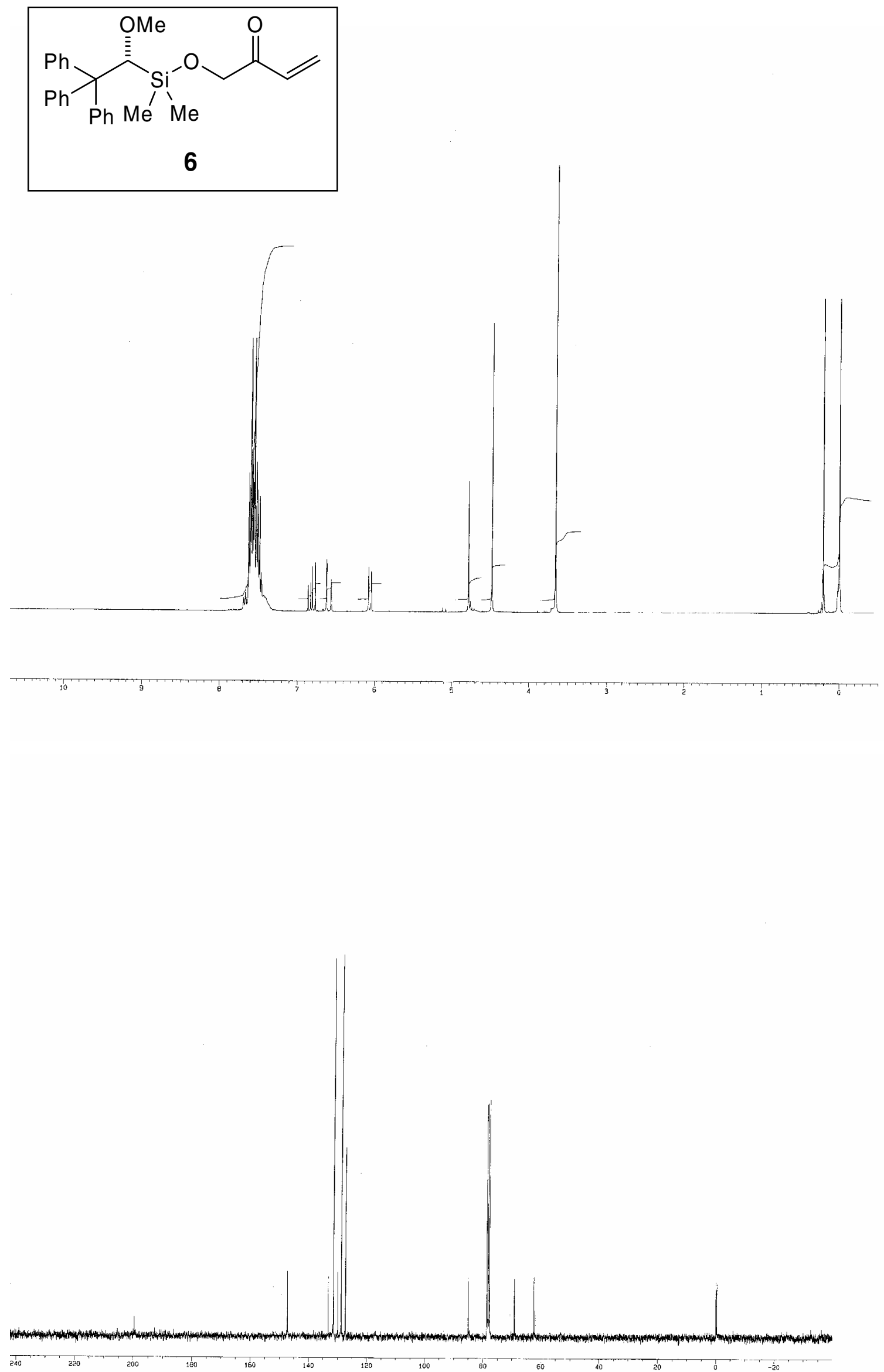

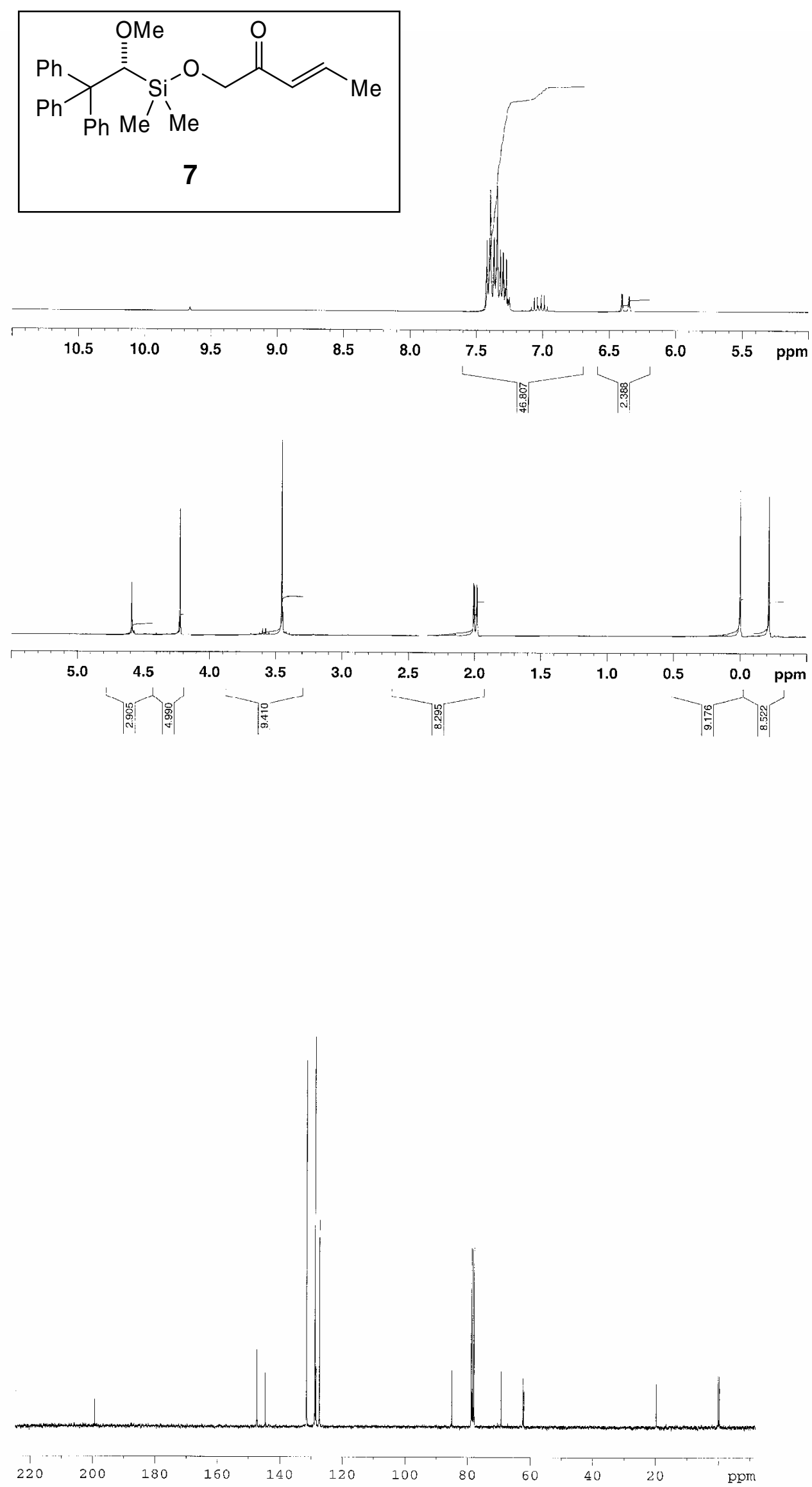


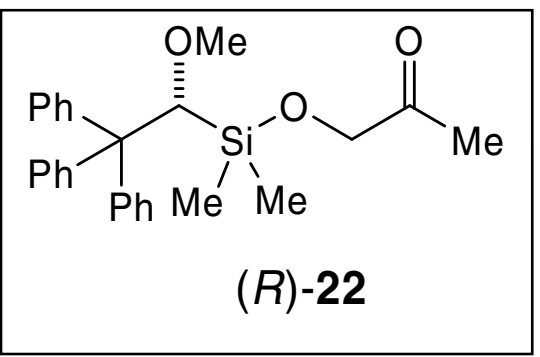
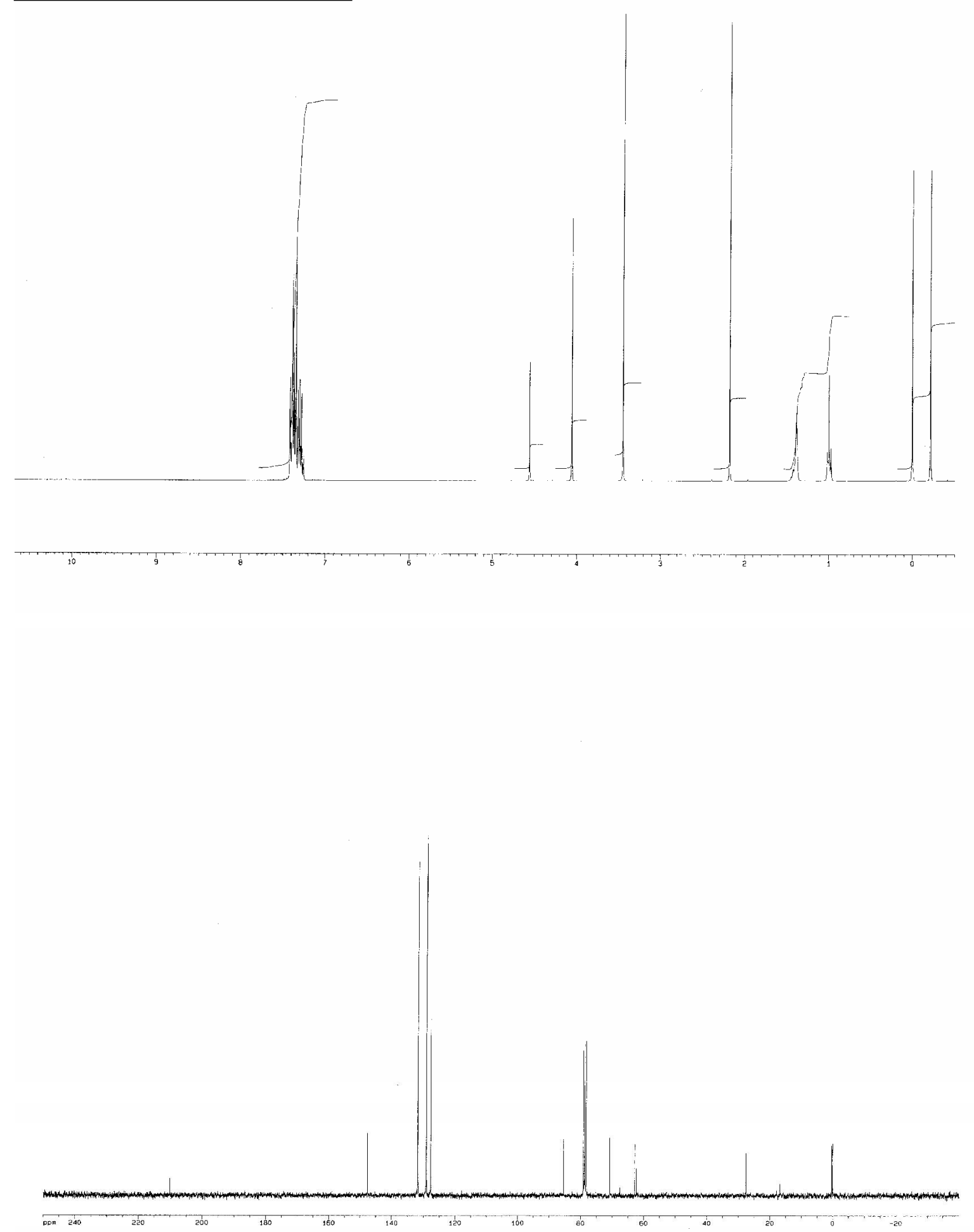


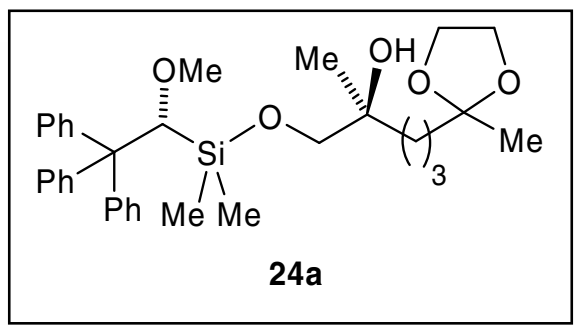

$-$

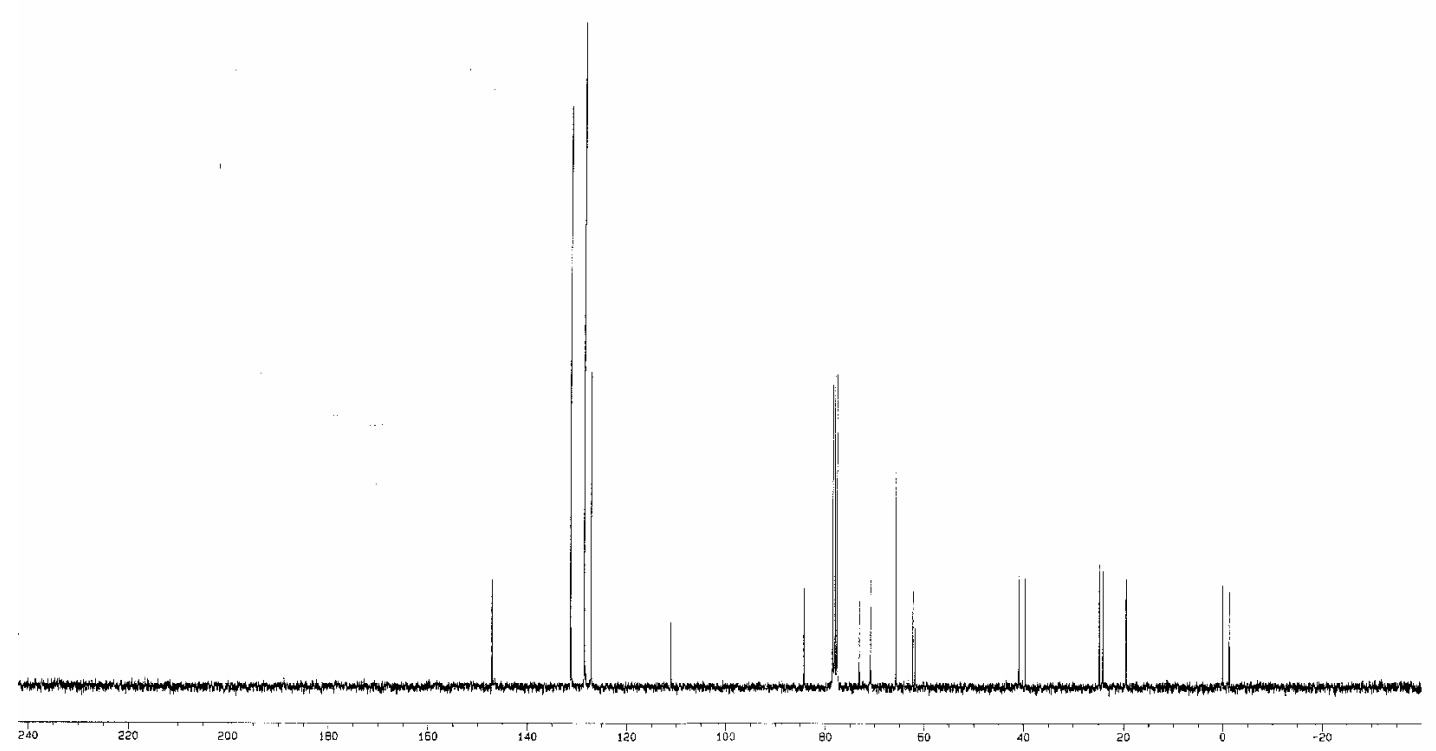



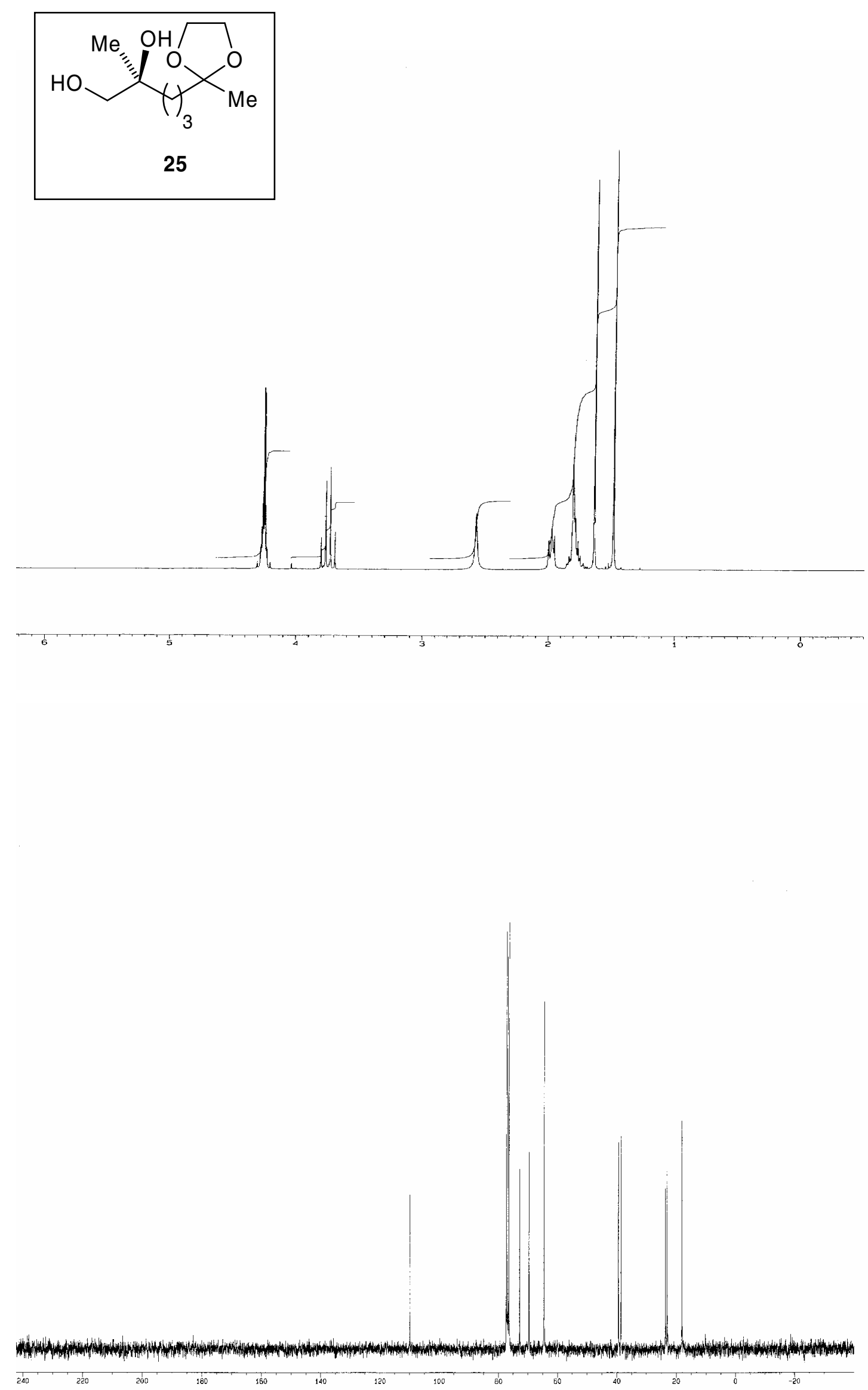
(-)-frontalin

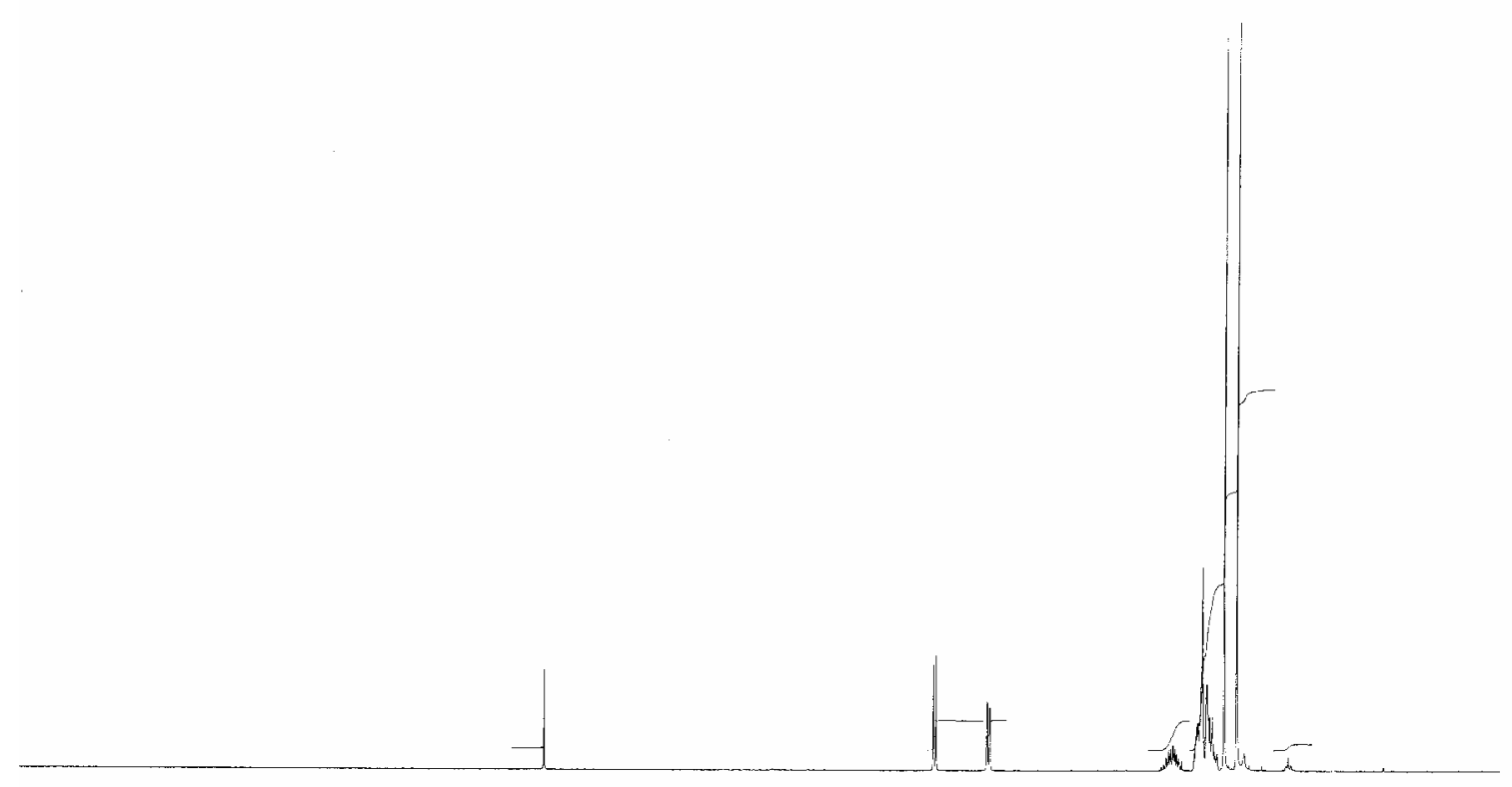

In the following page, HPLC analysis of a scalemic mixture of (+)- and (-)-frontalin (a) and sample after our enantioselective synthesis $(b)$. 

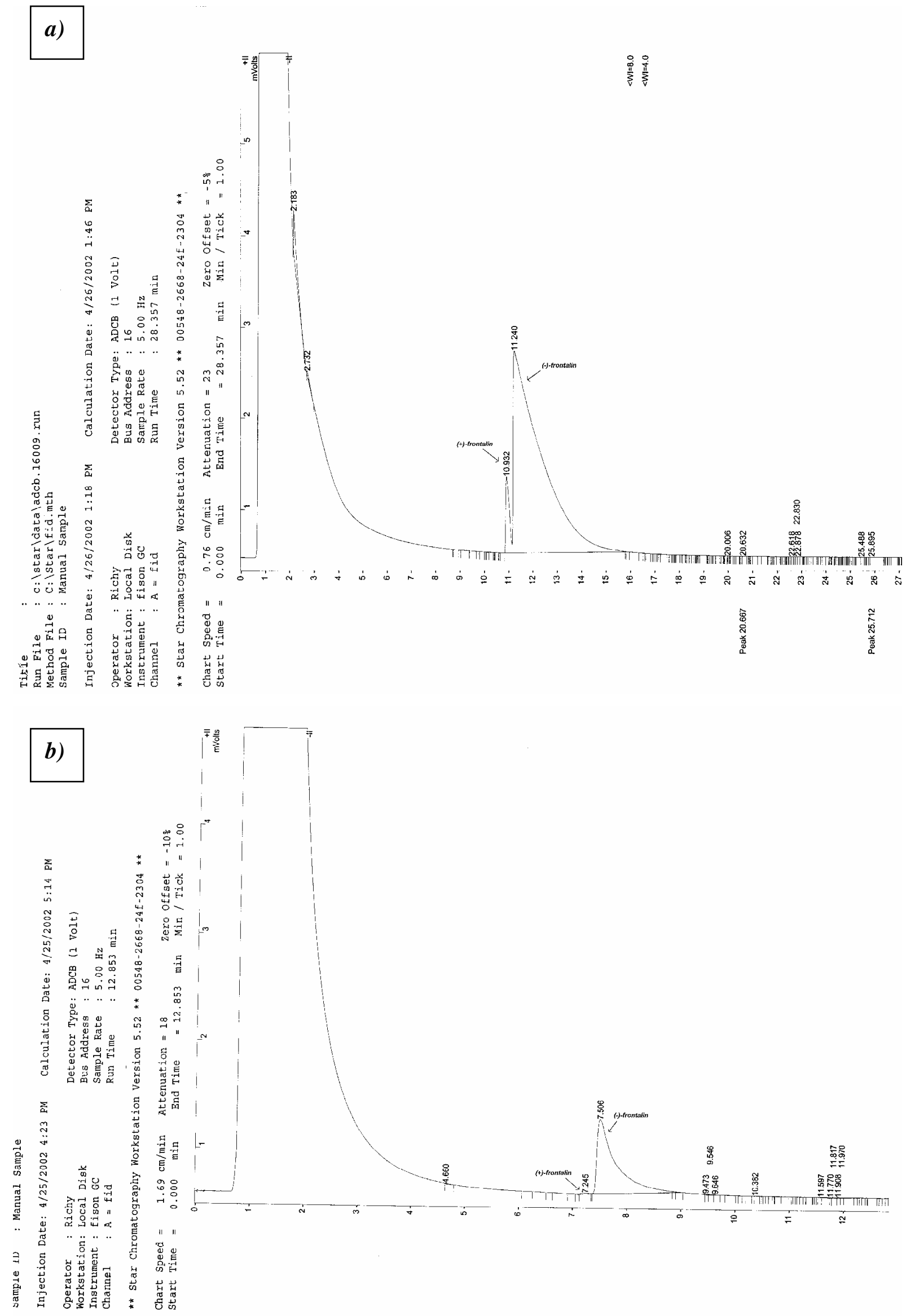

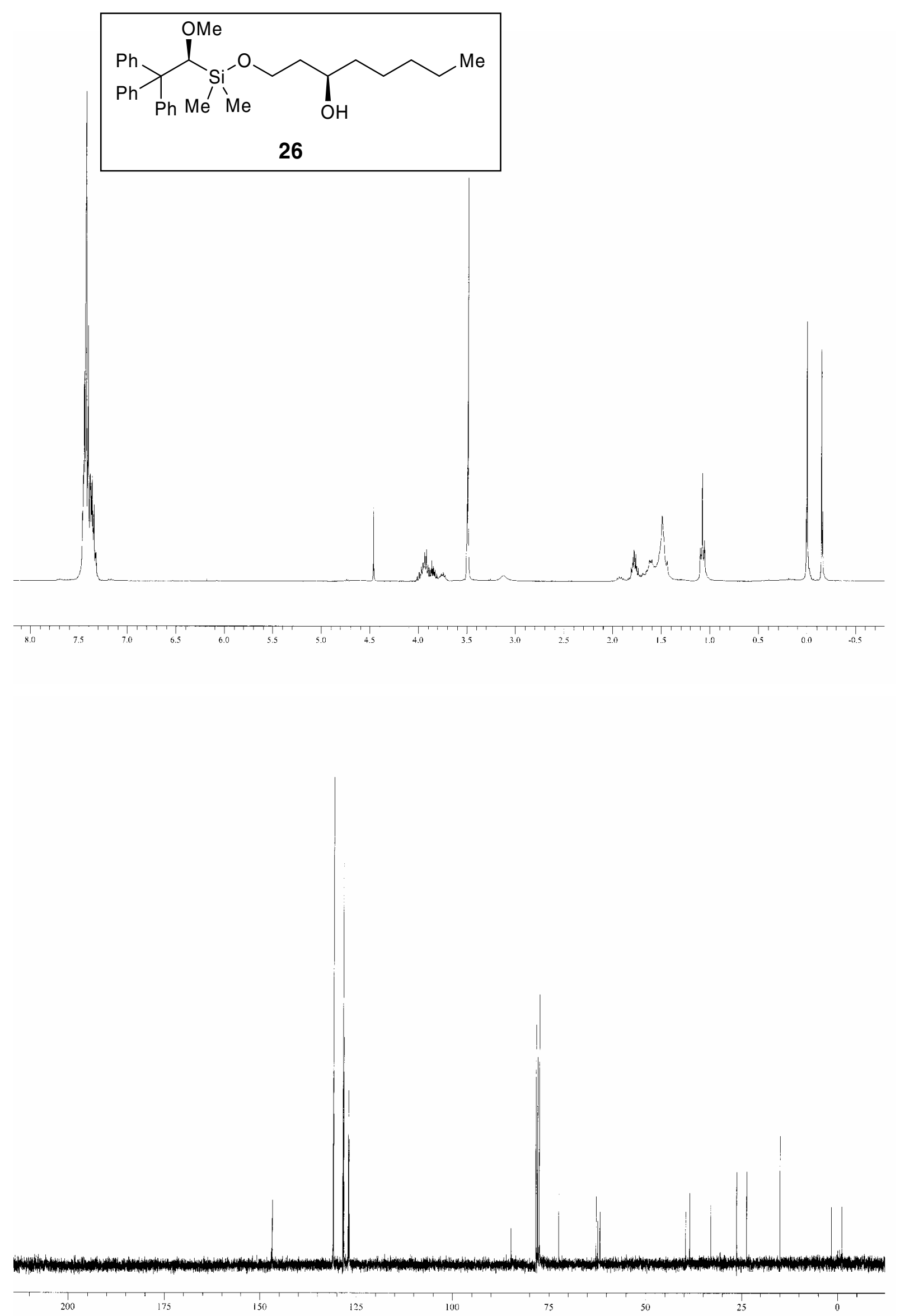

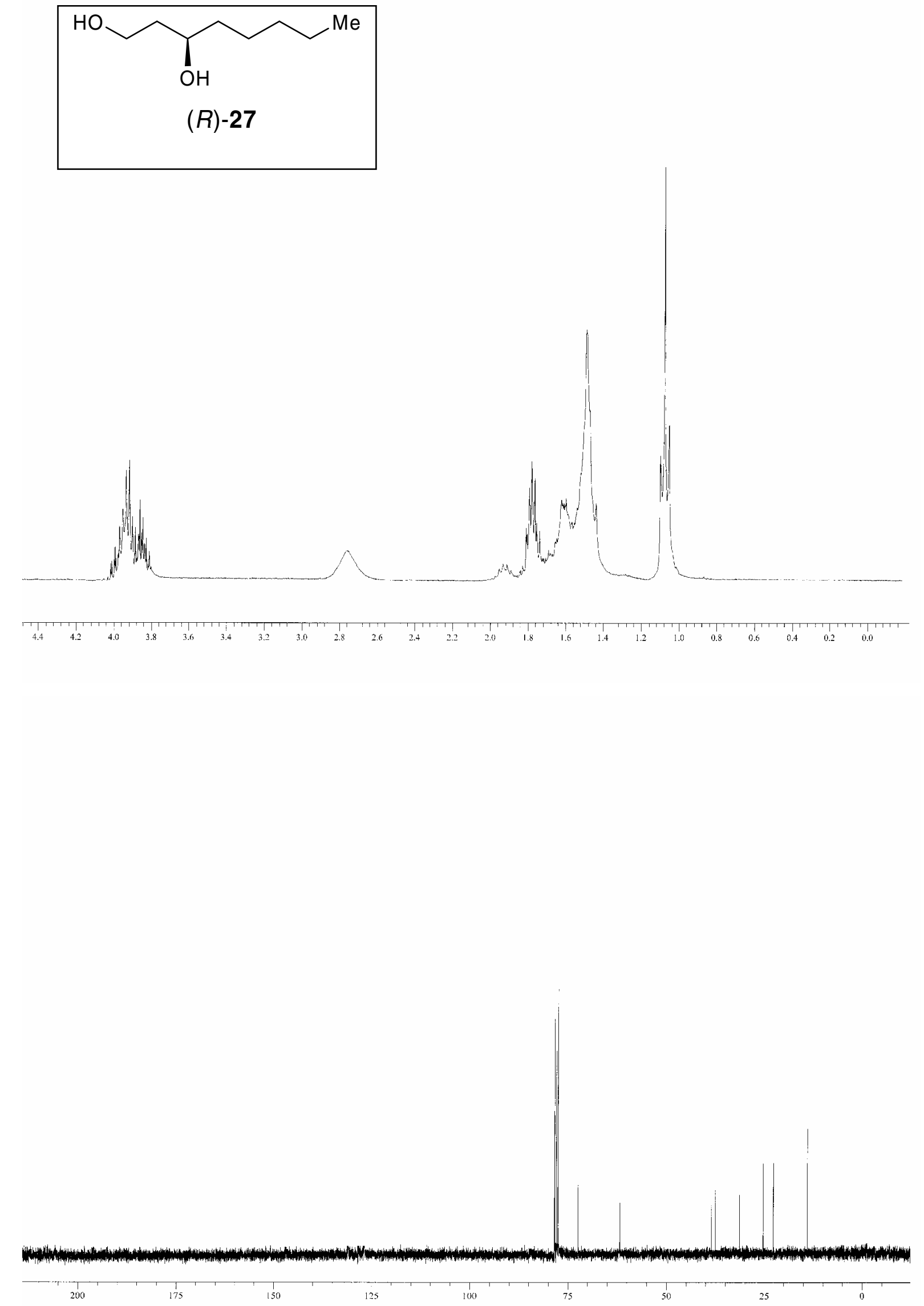

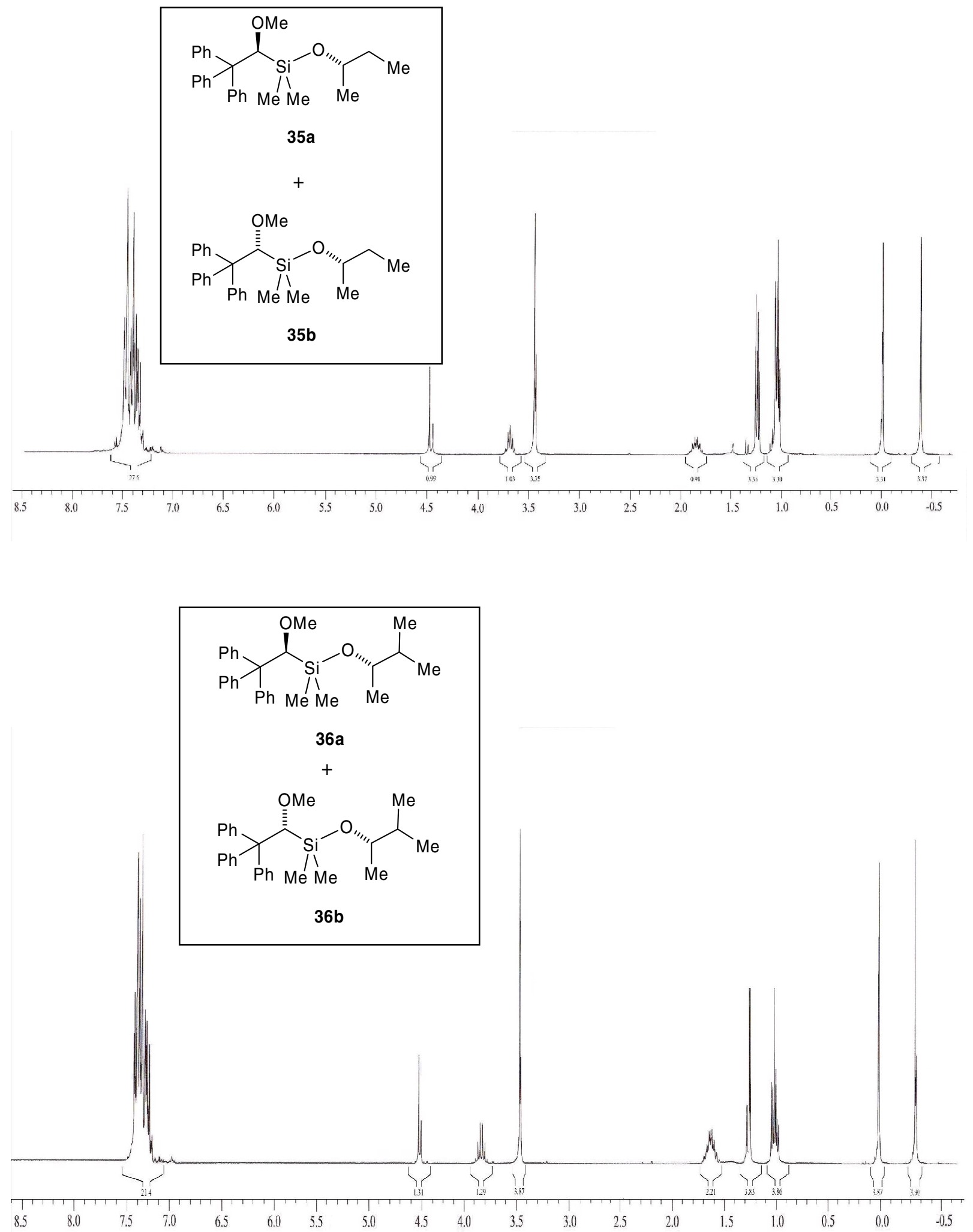

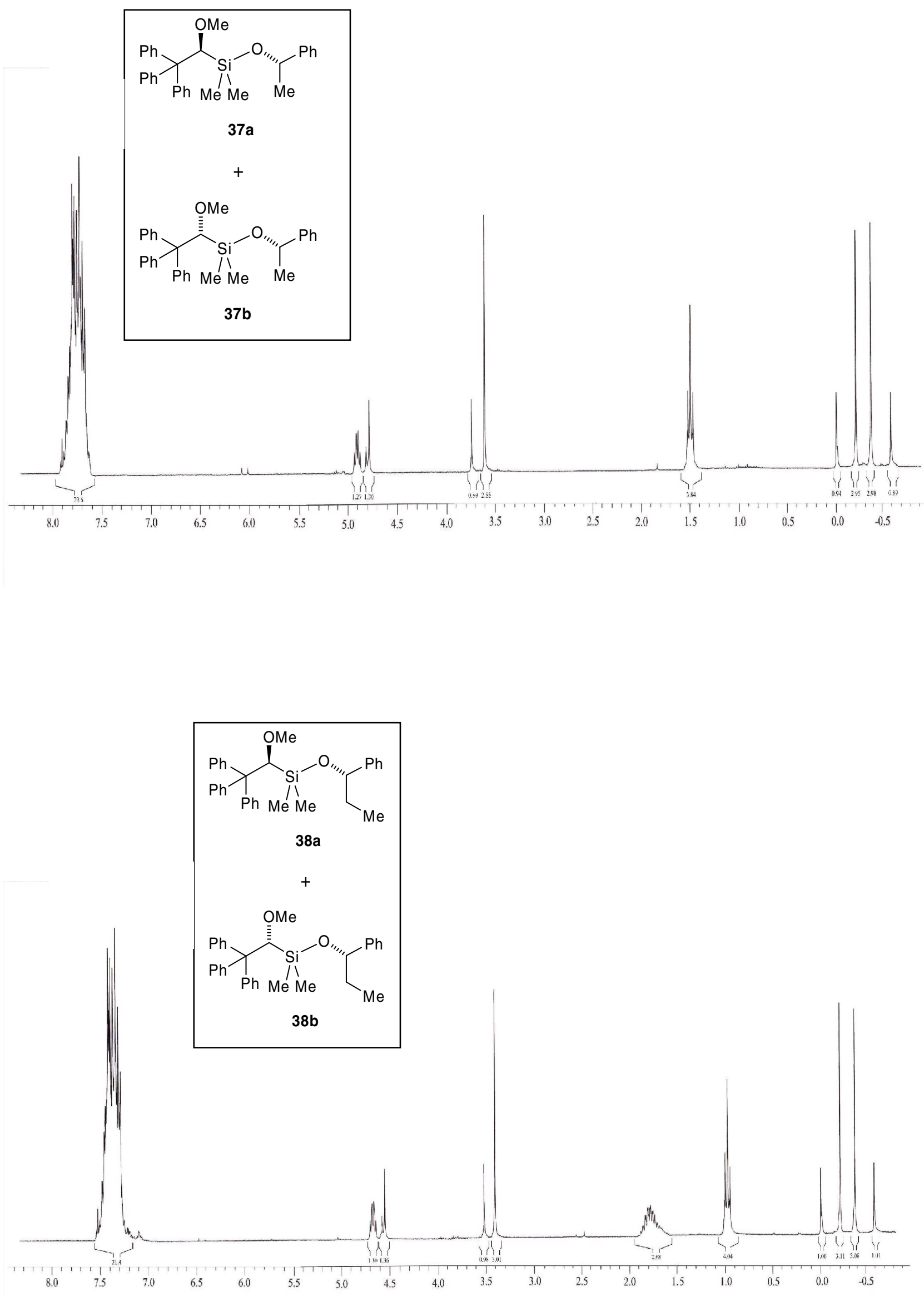

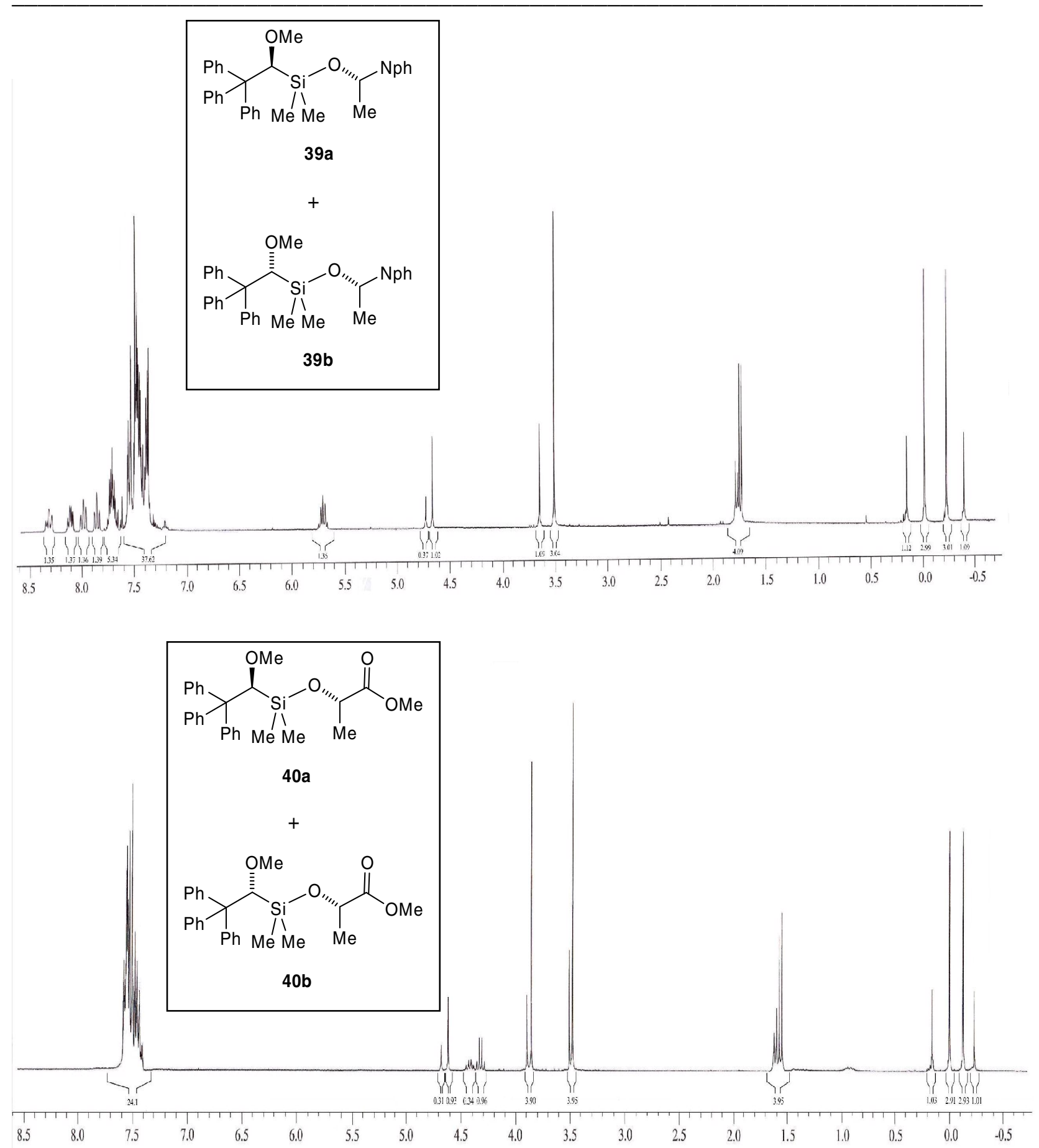


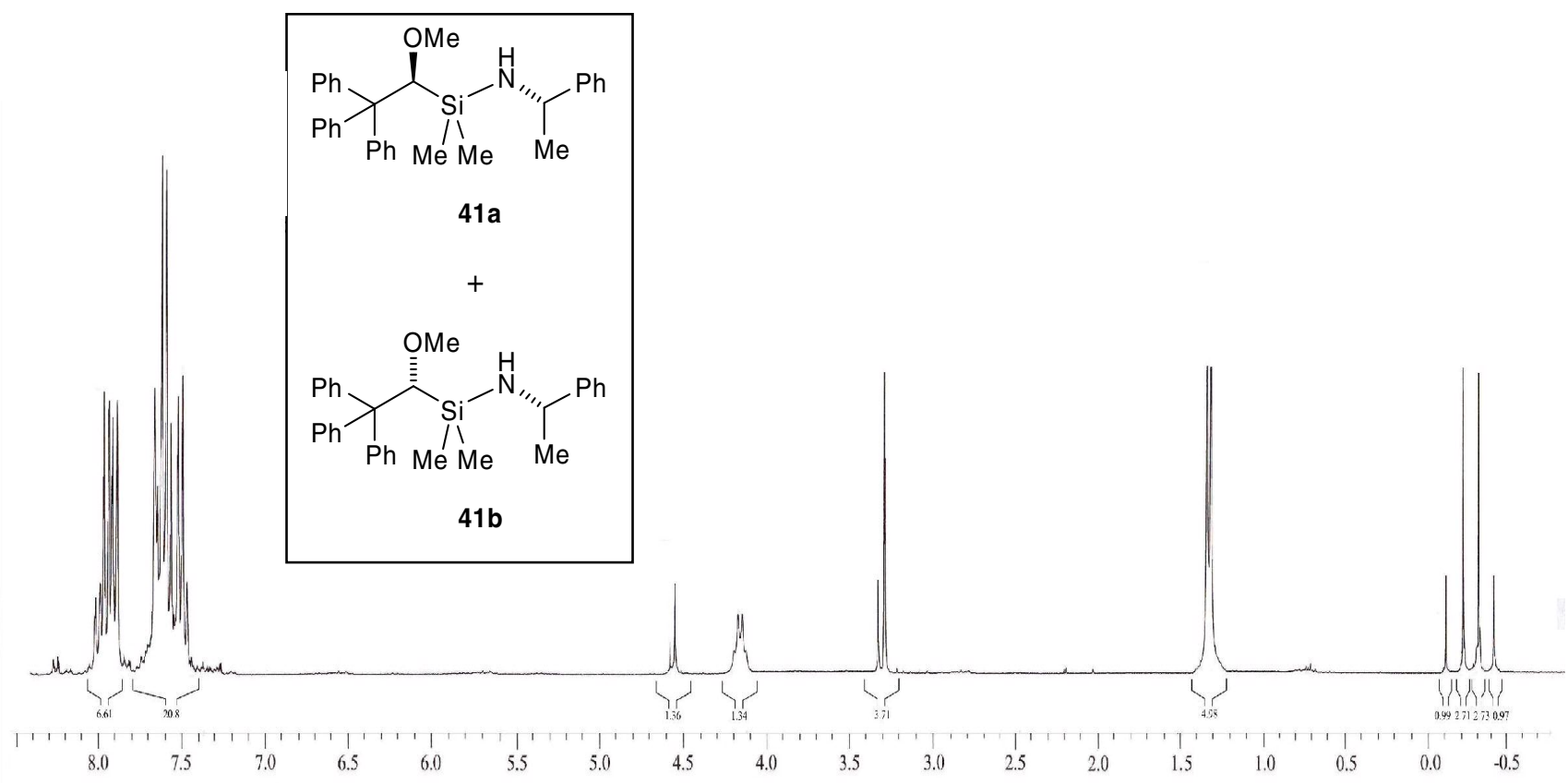

